\title{
exploration of the solar system
}

\section{(NASA-EP-122) EXPLORATION OP THE SOLAR}

SYSTEM (NASA)

72 P MF $\$ 2.25$; SOD HC $\$ 2.05$

N75-21307

CSCL $01 \mathrm{~A}$
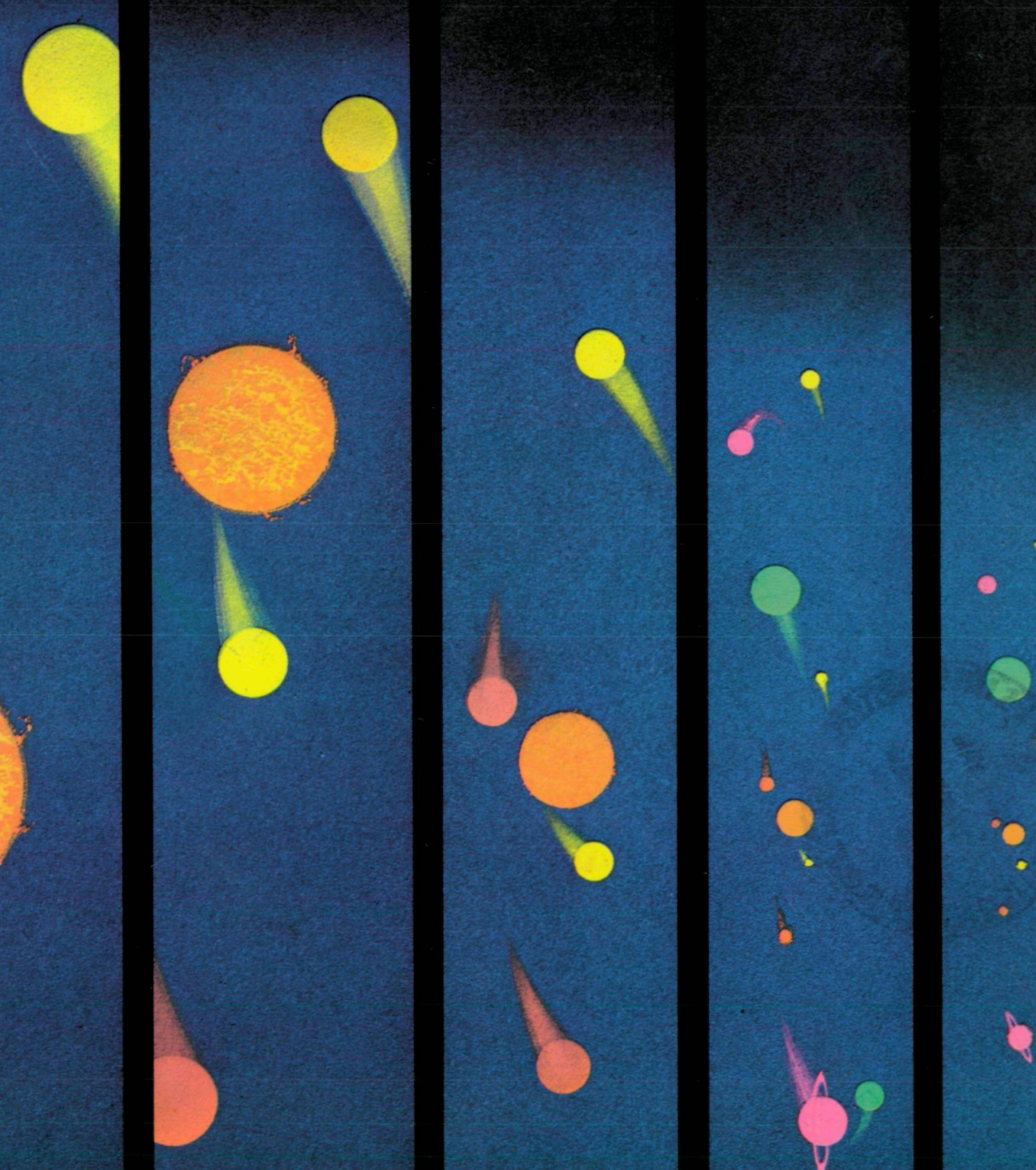

$\circ$

9 


\section{exploration of the solar system}

EP.122
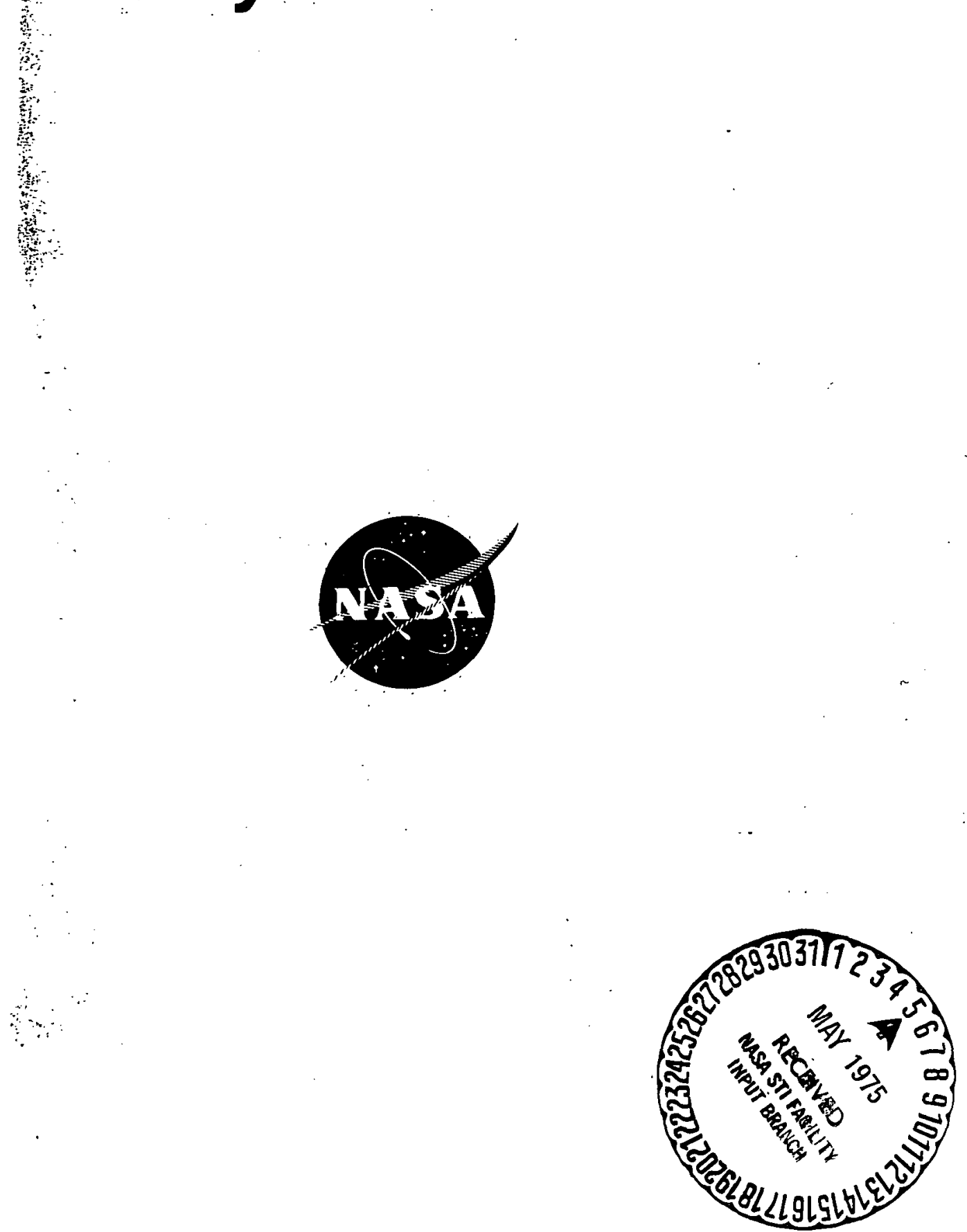

Prepared by Members of the AIAA

Technical Committees on Space Systems and Space and Atmospheric Physics

Edited by

Arthur Henderson, Jr., and Jerry Grey
Reprinted with permission from American Institute of Aeronautics and Astronautics 1290 Avenue of the Americas New York, New York 10019 


\section{American Institute of Aeronautics and Astronautics \\ Contributors to the Review of \\ Solar System Exploration \\ 1973}

Review Chairman

ARTHUR HENDERSON, JR.

Office of Aeronautics \& Space

Technology, NASA

(AIAA *TC-Space Systems)

\section{Contributors}

GEORGE R. CARRUTHERS

Naval Research Laboratory'.

JOHN R. CASANI

Jet Propulsion Laboratory

MURRAY DRYER

National Oceanic \& Atmospheric Administration

ROLF FAYE-PETERSEN

Wallace Murray Corporation

GEORGE J. GLEGHORN

TRW Systems Group

GEORGE HAZELRIGG

Princeton University

RONALD MASSA

Dynatrend Inc.

BERNARD P. MILLER

Econ Systems Inc.

GEORGE A. PAULIKAS

Aerospace Corporation

JAMES B. WEDDELL

Rockwell International

Space Division

HAROLD WHEELOCK

Jet Propulsion Laboratory

RAYMOND S. WILTSHIRE

Martin Marietta Corporation
Staff

JERRY GREY

CHRISTINE KROP

AIAA Headquarters

TC-Space \& Atmospheric Physics

TC-Space Systems

TC-Space \& Atmospheric Physics

TC-Space \& Atmospheric Physics

(Chairman, 1973)

TC-Space Systems

AIAA Member

TC-Space Systems

(Chairman, 1973)

TC-Space Systems

TC-Space \& Atmospheric Physics

TC-Space \& Atmospheric Physics

AIAA Member

TC-Space Systems

"TC: AIAA Technical Committee 


\section{Foreword}

The American Institute of Aeronautics and Astronautics (AIAA) is the technical society which represents a major segment of the aerospace profession's engineers, scientists, and students. The pooled technical knowledge of its 26,000 members encompasses all elements of the space field, including the technology of solar system exploration. It is our intent to make this knowledge available to whoever needs or wants it.

This Review is one of a series of Assessments and Reviews prepared in the public interest by the AIAA. These documents are written and assembled by ad hoc committees of volunteers drawn principally from the Technical Committees of the Institute under the overall guidance and supervision of the Technical Activities Committee, and with the assistance of the AIAA Headquarters staff. Each of these publications, including the present Review, is approved for public issue by the AIAA Board of Directors.

Contributors to this Review are identified on the facing page and in the Editor's note below. I ex- tend to them on behalf of all the Institute's members our most grateful thanks for the thousands of non-compensated hours they spent, as professionals in the aerospace field, to generate the material between these covers.

The information contained in this Review should be considered in the light of the following statement, issued with relevance to a previous AIAA Assessment by $\mathrm{my}$ predecessor as Vice President, Technical Activities and recent President of the AIAA, Dr. Holt Ashley:

"...Many shades of viewpoint exist among the contributors to this (Review), the elected officers, and the membership of the AIAA. Accordingly, we cannot expect universal agreement with every statement and conclusion. The broad comprehensive support it has received during the review process indicates, however, that the final product constitutes a fair consensus from a group of informed professionals.

"...We hope it will find wide usefulness in the process of decision making which affects.the future course of mankind's ventures in space..."

Starr J. Colby

Vice President,

Technical Activities

30 January 1974

\section{Acknowledgments}

The efforts of the contributors listed on the facing page in preparing and editing the material contained in the Review are gratefully acknowledged. Significant contributions were also made by a number of other AIAA members, directors, and officers during the extensive review process, particularly Pier Bargellini and Gary D. Gordon of COMSAT Laboratories; Rowland E. Burns, J. Reynolds Duncan, Jr., W. Haeussermann, and Ernst Stuhlinger of NASA's Marshall Space Flight Center; Louis D. Friedman, George Goranson, John Lucas, and C. W. Snyder of the Jet Propulsion Laboratory; Gordon Dugger of the Johns Hopkins University Applied Physics Laboratory; Grant Hansen of the General Dynamics Corporation; Leonard Jaffe and Robert S. Kraemer of NASA Headquarters; muel Katzoff and Eugene Love of NASA's Langley Research Center; O. K. Moe of McDonnell Douglas Astronautics
Company; and H. E. Rauch of Lockheed Missiles and Space Company, and S. T. Wu of the University of Alabama. AIAA staff members Leon Auerbach, Stanley Beitler, Johan Benson, James J. Harford, and especially Christine Krop provided invaluable assistance.

The editors wish especially to acknowledge the untiring efforts of three Contributors listed on the facing page: George R. Carruthers, John R. Casani, and George J. Gleghorn, who were principally responsible for assembling the bulk of the information appearing in Chapters 3, 4, and 5 . However, responsibility for the accuracy of this AIAA Review of Solar System Exploration rests solely with us, and although we recognize that despite our careful efforts some errors will certainly be found, we believe that the information in this document can be of service to the AIAA and to the nation.

Arthur Henderson, Jr. Jerry Grey 30 January 1974 


\section{TABLE OF CONTENTS}

I. Conclusions 1

II. The Purpose of Solar System Exploration _. 2

III. Our Knowledge of the Solar System __ 7

IV. Technology 27

A. Tools of Exploration

B. Spacecraft

C. Launch Vehicles and Propulsion

D. Advanced Technology Opportunities

V. Strategy for Solar System Exploration 43

A. Objectives

B. Mission Modes and Opportunities

C. Technological Constraints

D. Economic Constraints

E. The Program

List of Illustrations 61

Glossary of Terms 63 


\section{Conclusions}

The purpose of this Review is to outline the potential achievements of solar system exploration and suggest a course of action which will maximize the rewards to mankind. A secondary purpose is to provide, under one cover, a sourcebook of information on the solar system and the technology being brought to bear for its exploration.

We believe that the information presented herein supports the following conclusions:

1. It is appropriate for the United States, as a technological nation, to establish a balanced national research program that assures continuity of scientific research in all areas of human understanding and that provides for an ever-widening horizon of technological opportunity.

2. Solar system exploration is a major scientific frontier that deserves a place of priority in a balanced program of scientific research.

3 . The extent to which this nation pursues scientific exploration of the solar system today will significantly affect its ability to pursue these endeavors in the future and to maintain pace with the other technologically advanced nations.

4. Solar system exploration has already provided some significant contributions to the solution of man's problems on Earth, but its principal impact will occur in the future, as a result of the knowledge and understanding which will be gained by exploring the basic phenomena of our Earth's environment.

5. Solar system exploration, because of its unique dependence on advanced technology and extremely long-range project planning, requires support on a long-term rather than on a year-to-year basis. Short-period fluctuations in budget allocations; e.g., over periods of half a decade or less, will not only result in serious losses of future potential options, but can also generate substantial waste of the nation's financial and technological resources.

A corollary to this principle of sustained funding is that the investment allocated to long-range research programs, whose impact can be felt only after time periods measured in decades, should not be subject to the same constraints (e.g., social discount rate) as are generally applied to shorter-range development or construction efforts requiring capital investment. Exploration of the solar system qualifies as such a long-range program. 


\section{The Purpose of Solar System Exploration}

In 1965 the National Academy of Sciences restated three broad questions whose answers were to be sought through space exploration:

(1) What is the origin and evolution of the universe, and specifically of the solar system of which Earth is a part?

(2) What is the origin, evolution and distribution of life, including life elsewhere than on Earth?

(3) How do dynamic processes and events occurring beyond the Earth modify man's terrestrial environment?

The questions are not new. Philosophers and scientists have contemplated them for centuries. What is new is the promise that space exploration can bring us closer to the answers, which will be important to our present security and our future development. A purpose of this document, therefore, is to explore the degree to which these three practical questions can be answered:

(1) Why must we explore the solar system?

(2) Why is understanding the solar system important to us? How does it relate to such contemporary issues as health care, transportation, energy, resource management, protection of the environment, war and peace, and others of similar import?

(3) Why can't we wait until all of these problems on Earth are solved before we try to solve problems in space?

(1) Why must man explore the solar system?

From the earliest times, man's efforts to understand the solar system have been fundamental to his technological progress and his view of his place in the universe. Recognition of the seasons of the year and their relation to the times and places of sunrise and sunset was basic to the development of agriculture. The planting and harvesting of crops led to the first fixed settlements, to cities and to nations. In ancient Egypt, measurements of the times at which certain stars rose were used to forecast the flooding of the Nile and the correct time for planting.
The association of the positions of the Sun, Moon and stars with seasons, tides, and floods gave rise to astrology - the belief that heavenly bodies govern men's lives. Because of the importance of astrology, the ancients made accurate observations, using the unaided eye and sighting circles, of the motions of the planets-those starlike objects that wandered through the seemingly unchanging pattern of the stars. By the time of Ptolemy, about 130 B.C., these observations had been built by the contributions of the ancient philosophers into a rather complicated theory in which the Earth was the stationary center of the universe, and the Sun, Moon, planets, and stars revolved around it. In the Middle Ages, the Ptolemaic theory became part of the dogmatic philosophy to which all educated Europeans were expected to adhere.

Copernicus (and others before him) challenged the Ptolemaic theory, maintaining that only the Moon revolved about the Earth, which rotated on its own axis and, together with the other planets, moved about the Sun.

As others began to question the established doctrines, there followed all the inventiveness, curiosity, expansion, and openness of mind that have distinguished modern from medieval civilization. In 1609, Galileo looked at the skies with. a telescope and confirmed the Copernican theory. He saw Jupiter as a globe with four moons circling it: a scale model of the solar system. He also was the first to see mountains and craters on the Moon, the crescent phases of Venus analogous to those of our Moon, and (first among Europeans) spots on the Sun.

Earlier, Galileo had been the first scientist to connect experiments with mathematics to explain the motion of falling objects here on Earth. It was now clear that a unifying principle must exist among celestial and terrestrial events. Isaac Newton established this principle in 1686 by publishing his laws of motion and gravitation. These laws were the beginning of modern physics and the basic tool of modern engineering.

In the mid-nineteenth century, another major advance took place. Fraunhofer's observations of the spectrum of sunlight and its identification with the spectra of chemical elements measured in the laboratory led to the recognition that the Sun and Earth contained the same substances. This knowledge helped open the door to modern chemistry and atomic physics.

These discoveries about the solar system have changed man's conception of his world and his place in it. Instead of the central figure in a mysterious and capricious universe, he sees himself as a small part of a nature he can hope to under- 
stand and perhaps in part to control. In recent years the use of space vehicles has made possible greatly increased understanding of the solar system. Spacecraft have placed telescopes above the Earth's atmosphere where they can observe radiation at all wavelengths, with all its information about planetary and solar atmospheres. They have placed detectors directly within the interplanetary gas. They have allowed photography of planetary surfaces at resolutions measured in hundreds of meters rather than hundreds of kilometers, and they have disclosed the interaction of some planets with the surrounding solar wind. The resulting gains in understanding of the universe have indirectly affected our daily lives in many subtle ways, and may be expected to influence our future progress substantially.

(2) Why is understanding of the solar system important to us, now and in the future?

Man's exploration of the solar system is often considered to be no more than an exercise in his intellectual curiosity, and of value only to further the understanding of his origins and to aid his purely intellectual development. This. view is, however, a limited one. As we have seen in the preceding paragraphs and as will be discussed further in some detail, from the work of the earliest astronomers, mankind has derived tangible benefit from such exploration in virtually all areas of human endeavor. And although the past is no guarantee for the future, it appears that today, with our advanced state of technology and manifest needs, the potential for such benefits is greater than ever before.

It must be made clear, however, that these potential benefits, like those of any research program whose time scales are measured in decades, cannot be subjected to the usual criteria employed in the analysis of benefits and costs. History has clearly marked the progress which is directly traceable to non-directed basic research, and it is this aspect of solar system exploration which constitutes its primary motivation.

Even at this early stage in solar system exploration, however, several potential benefits are beginning to surface. For example, solar radiation is not only the source of all life (as well as a danger to life) but also is one of our two principal potential sources for future energy supplies. Furthermore, the Sun has served as a model for many of our other energy-source developments. The basic concept for nuclear fusion, our other principal potential source of energy, was derived originally from studies of the Sun, as will be discussed later.

Disturbances in the solar wind, as produced by solar flares, cause corresponding disturbances in the Earth's magnetosphere (magnetic storms). These storms are responsible for communications disruptions as well as brilliant auroral displays. They can be quite hazardous to astronauts, but may also affect aircraft crews and passengers. We need to understand more about these spectacular phenomena. Statistical studies suggest that magnetic storms may appreciably affect global aspects of our terrestrial weather. Thus, monitoring of the solar wind may become an essential element in complete terrestrial weather forecasting.

The ultraviolet and X-rays emitted by the Sun are responsible for the terrestrial ionosphere, a phenomenon essential to long-distance radio communication. Another vital product of solar ultraviolet radiation is the terrestrial ozone layer. Ozone has the important property of absorbing ultraviolet light and preventing it from reaching the surface of the Earth. Without this protection, life as we know it would be very difficult or impossible. Thus, it is of great practical importance that we understand variations in solar ionizing radiation.

The planets of the solar system are natural laboratories for observing the extension of the ranges and scales of phenomena also present on Earth. For instance, exploration of the trapped radiation and magnetic fields of other planets is an important tool in understanding the formation and development of all planets, including our own. This understanding may bear upon problems of preserving Earth's water supply and controlling the long-term development of our atmosphere.

Further, man cannot ignore the possibility that he may require the use of the uninhabited planets as cosmic laboratories to conduct experiments and operations necessary for his survival but far too dangerous to conduct on Earth. For example, we are already considering using the Sun or interplanetary space to dispose of future radioactive wastes from nuclear powerplants.

We can ask many even more specific questions in solar system exploration whose answers are directly applicable to Earth interests. Highly accurate distance measuring on interplanetary scales is currently being applied to intercontinental surveying on Earth. This research is the basis for study of the mechanics of earthquakes and volcanic' activity. Stérilization techniques originally developed for planetary quarantine have so far surpassed conventional methods that they are being rapidly incorporated by large general hospitals across the country, and are beginning to contribute to the success of sophisticated medical procedures-just 16 years from the first space launch. The concepts of reliability and miniaturization, needed to make long deep-space missions a reality, are 
already at work for us in radios, television sets, computers, communication devices, and innumerable other applications.

In a few years the impetus from development of our outer-planet spacecraft, faced by communication turnaround times measured in hours, will make "self-repairing" mechanical/electrical systems feasible. These spacecraft will also have to incorporate enough "artificial intelligence" to enable them to react to events requiring near-instant decisions. Such systems will have considerable potential for improvement of our Earthbased transportation and communications systems.

Although many of the "visible" "benefits of solar system exploration are in these indirect "spinoffs," we must never lose track of the basic truth that they are not by any means the sole or even the main justification for space spending. As Senator Lowell Weicker of Connecticut has warned us:

"Much of the emphasis of the space program lately has been pointed toward Earth's benefits of the program. Fair enough; in a time of lagging interest, it's proper to remind the public of the tangible benefits that have spun out of the space program. But let's remember one thing: the space program did not set out to find these specific offshoots. They emerged and were seized on as part of the more difficult, almost impossible, effort to get on the Moon. And so we can cite such accomplishments as the communications satellites and the special weather-forecasting satellites, ind isputable great boons to mankind. But remember, we came upon them on our way to the stars-not by grubbing around in the ledgerbooks."

And it is "on our way to the stars" that the greatest benefits to mankind will be reaped. First, from an international viewpoint, the solar system belongs to all men, and man's common goals for its exploration have served to unite nations in ways that can only benefit all concerned.

In a sense, science, like the arts, offers an opportunity for cultural exchange-a common ground for men to meet in cooperation rather than conflict. One principal bonus of cooperation 'in international space exploration is the relief of the binding tie in formal or informal treaty arrangements. International cooperation also paves the way for a certain amount of technological cross-fertilization and elimination of duplication of effort. These factors associated with the involvement of other nations help to justify continuation of specific programs. The current involvement of the European Space Research Organization in developing a Spacelab to be used with the U.S. space shuttle is a case in point.

There also is a part to be played by competition. Man, as sociologists are rediscovering, is still a contentious, envious creature: W.ithout competition. his projects may lose their spark and drive. Much of our technological progress in the past. with its consequent impact on the dominant indus trial and economic factors which govern our present civilization, has been made through war. The exploration of space offers a far more attractive motivation for broad advances in technology, particularly if a proper balance can be struck between international cooperation and a healthy rivalry in space exploration achievements.

Also, space exploration brings our goal-seeking youth new horizons to be explored; new areas to occupy and exercise their intellects. Finally, a fundamental drive of man in his exploration beyond the Earth is the search for extraterrestrial life.

At present, there is no direct evidence for life on any of the other planets, but the observations to date would hardly be capable of detecting life on Earth. It has been said that if life of any kind is discovered elsewhere in our own solar system, we can assume that it will occur wherever conditions amenable to the origin of life exist-and, hence, that intelligent civilizations probably exist elsewhere in our galaxy. Nothing could have a greater impact on man, his philosophy or his institutions than the discovery of intelligent life elsewhere, and the subsequent need to cope with it.

It is in such a framework that the motivations for solar system exploration should be viewed, rather than in the immediate "marketplace" of cost and benefit comparisons with other critically important but necessarily short-range national needs.

(3) Why can't we wait until all of our problems on Earth are solved before we try to solve problems in space?

This is perhaps the most difficult of the three questions to answer specifically and directly. Clearly, there is never enough financial support for all worthy programs, and our national budgeting process, just as in our everyday household or business budgets, consists of a balancing of needs. Economists attempt to do this by using formal benefit/cost analyses; that is, programs with the most benefit per unit cost should receive priority. But how. do we quantify future, mainly unidentified benefits? Or costs, for that matter, if we include the actual costs of national resource depletion or environmental degradation?

The scientific community is often concerned because there are frequently insufficient long-term commitments to scientific programs. However, scientists sometimes do not understand that the ultimate source of support is the voting taxpayer, and most voting taxpayers do not understand 
what the scientist wants to do or why he wants to do it.

The following excerpt from the Hearings before the Subcommittee on Space Science and Applications of the Committee on. Science and Astronaurics. House of Representatives, Ninety-Third Congress. March 14, 1973, illuminates this point:

Congressman James Symington. I suppose that all of us feel the constraints of the current financial situation. Harder decisions are being made now than may have been the case 19 years ago, but that doesn't make them correct, just because they are hard.

When you say the decision was not made by NASA alone, or the fault was not that of NASA alone, and the blame belongs elsewhere, at least partially, I take it you're referring to the managers of the budgetary process. Now they probably are trying to think of what the market will bear in terms of public acceptance of programs, or perhaps to put it another way, what we will least notice is missing.

What I think our task may be, with your help, is to try to convey to the average citizen what would be missing if-we failed to go forward with these projects.

You have described the concern of the scientific community in the suspension of the HEAO (High Energy Astronomical Observatory) project, but that concern obviously is not widely shared, as the average citizen knows nothing of it.

What am I to say to my constituents and people I speak to concerning the importance of this program in language, they will understand?

Dr. Hofstader.* I think that's a very good question... Let me give you an example. When Einstein made his considerations about mass, and energy, and relativity, it looked like they were completely academic and had nothing to do with anything practical, but we know how important those things are. Those are the foundations of atomic energy.

The neutron was discovered in 1932. It's only 40 years since the discovery of the neutron. Now we have the whole atomic energy industry. Even as long ago as in the late 1930's or 1940's, Rutherford himself said that the neutron would never have any practical signifiance.

What I'm trying to say here is that scientists who are working with these abstract ideas, which apparently have no relationship to the real world, suddenly find that they are terribly, terribly important, and I think this is going to happen again... There's going to be a new source of energy

*Dr. Robert Hofstader, Department of Physics, Stanford University. that's going to turn the whole world around..."

In today's "show me a result" atmosphere, the often long and arduous "scientific process" does not appear to generate much that people wish to buy; the man in the street finds it difficult to relate to, say, quantum mechanics. What do scientists have that we should want? C. P. Snow wrote in Science and Government of the contributions that science made to Britain's survival in World War II. He said, "Scientists have something to give which our kind of existential society is desperately short of; so short of that it fails to recognize of what it is starved: that is foresight."

It is that kind of foresight, for instance, that led knowledgeable scientists and engineers to predict many years ago the approach of our current energy crisis.

Scientific "foresight" was a key element in the potentially valuable discovery made by physicist Dr. Hans Bethe, when in 1933 he published his theory on the energy conversion process which powers the Sun. Although of enormous importance to the scientific world (Dr. Bethe won the Nobel prize for his work), there seemed little practical application of such knowledge here on Earth-until the energy crisis came along. The science and technology of nuclear fusion, perhaps the best hope for Earth's long-range energy needs, originated in Dr. Bethe's "abstruse"' astrophysical theories.

More recently, a team of dedicated men discovered a polio vaccine. But their discovery would not have been made possible without the electron microscope, a tool which had been developed for purposes totally unrelated to polio research.

Clearly, well thought out long-range planning is required to maintain the Earth in a livable state. This planning must be on a global scale, and must include consideration for all peoples. This point was well-illustrated by Dr. Ernst Stuhlinger in his letter to a nun working with the starving people of Zambia, Africa:

"About 400 years ago, there lived a count in a small town in Germany. He was one of the benign counts, and he gave a large part of his income to the poor in his town. This was much appreciated because poverty was abundant during medieval times and there were epidemics of the plague which ravaged the country frequently.

"One day, the count met a strange man. He had a workbench and a little laboratory in his house, and he labored.hard during the daytime so that he could afford a few hours every evening to work in his laboratory.

"He ground small lenses from pieces of glass; 
he mounted the lenses in tubes, and he used these gadgets to look at very small objects. The count was particularly fascinated by the tiny creatures that could be observed with the strong magnification and which nobody had ever seen before.

"He invited the man to move with his laboratory to the castle, to become a member of the count's household and to devote henceforth all his time to the development and perfection of his optical gadgets as a special employee of the count.

"The townspeople, however, became angry when they realized that the count was wasting his money, as they thought, on a stunt without purpose. 'We are suffering from this plague,' they said, 'while he is paying that man for a useless hobby!'

"But the count remained firm. 'I give you as much as I can afford,' he said, 'but I will also support this man and his work, because I know that some day something will come out of it.'

"Indeed, something very good came out of this work, and also out of similar work done by others at other places: the microscope. It is well known that the microscope has contributed more than any other invention to the progress of medicine, and that the elimination of the plague and many other contagious diseases from most parts of the world is largely a result of studies which the microscope made possible.

"The count, by retaining some of his spending money for research and discovery, contributed far more to the relief of human suffering than he could have contributed by giving all he could possibly spare to his plague-ridden community."

The direct benefits to man of the knowledge gained and the tools developed as a consequence of man's exploration of the solar system are only beginning to be recognized. More are still to come. But at what cost?

NASA's budget for 1974 is $\$ 3$ billion, of which about $\$ 350$ million is to be directly applied to solar system exploration projects. The total federal budget is $\$ 269$ billion, of which $\$ 25$ billion is for interest on the national debt, $\mathbf{\$ 8 1}$ billion is for defense, and about $\$ 126$ billion is for human resources.

Thus the lion's share does go to societal concerns, as it should. At the same time, the third of a billion dollars that is slated for solar system exploration (13 hundredths of one percent of the total budget) is indeed a small investment in the future. As Lawrence Lessing wrote in FORTUNE in 1964:

"The purposes of this (space) exploration are no clearer to many men in this age than they were in Galileo's, so it is not strange that there is opposition. In this economic age, however, the opposition is not so much theological as budgetary. Both seem equally mistaken in the context of their times, for the earlier astronomical discoveries did not diminish man's spirit but rather enlarged and ennobled it, and space discoveries should have the same uplifting and enlarging effect. After all, a budget . is only money, but new knowledge is a dukedom : whose great wealth and resources cannot even begin to be estimated or exhausted. Aleady the new knowledge acquired in space exceeds by far the value of funds so far spent. For knowledge, more than guns or butter, is the true power of modern states." 


\section{Our Knowledge of the Solar System}

The advent of space experimentation has resulted in a large jump in our knowledge of the solar system, especially of the nearest planets, Mars and Venus. To place this knowledge in perspective, we will first consider some characteristics of our Earth. Here we emphasize discoveries made by artificial Earth satellites and show how these discoveries carry out the exploration of the solar system of which our planet is a member.

\section{EARTH}

The Earth has a molten core of iron and other metals, surrounded by a hot, solid mantle of silicates and oxides of light metals. Above the mantle is the crust, which contains the mineral resources available to man.

The Earth is unique among the planets of the solar system in its atmospheric composition $(78 \%$ nitrogen, $21 \%$ oxygen, $1 \%$ argon and other gases). This atmosphere, as well as those of Mars and Venus, is believed by some to have resulted from outgassing of the interior (by volcanic activity, for example) after it was formed, rather than by gravitational capture from its original solar nebula, as win the case of the giant outer planets Jupiter, Saturn, Uranus, and Neptune. Green plants on Earth, which break down carbon dioxide to form - oxygen, have also contributed to the Earth's atmospheric oxygen content.

The ozone layer is centered near 25 kilometers (15 miles) altitude. Ozone is a form of oxygen containing three atoms per molecule $\left(\mathrm{O}_{3}\right)$ instead of two as in ordinary oxygen $\left(\mathrm{O}_{2}\right)$. It is formed by subjecting ordinary oxygen to solar ultraviolet light. At still higher altitudes, most oxygen is found in the atomic form $(\mathrm{O})$ rather than the molecular form $\left(\mathrm{O}_{2}\right)$ or ozone $\left(\mathrm{O}_{3}\right)$.

At altitudes greater than $100 \mathrm{~km}$ (62 miles), the atmosphere contains many electrically charged particles (electrons and ions). This region is known as the ionosphere. An ion is an atom from which one or more electrons has been removed (or added) producing a net positive (or negative) charge. In the upper atmosphere, the ionization is produced mainly by ultraviolet and X-rays emitted by the Sun. It is vital to many means of long distance communication. Without it, most radio communication would be limited to line-of-sight distances (up to about 100 miles) rather than around-theworld as is possible now.

At altitudes above $1000 \mathrm{~km}$ (620 miles) the atmosphere consists mainly of the light gases hydrogen and helium. This region, known as the exosphere, or geocorona (see Fig. 1); is important in our search for understanding of the origin and evolution of our atmosphere, because hydrogen and helium, which are too light to be permanently retained by the Earth's gravity, must be continually replaced from some source. The hydrogen is believed to be produced mainly by the breakup of water vapor molecules in the upper atmosphere, caused by solar ultraviolet light (photodissociation). The oxygen is retained by the Earth's gravity, whereas the much lighter hydrogen eventually escapes. This process is believed to have been the main source of oxygen in the atmosphere before the advent of life and green-plant photosynthesis. The helium in the atmosphere is produced by the decay of radioactive materials, such as uranium and thorium. Argon is produced by the decay of a radioactive form of potassium. Being relatively heavy, argon does not escape, but builds up in the atmosphere. There is a puzzling discrepancy in the helium concentration: it is only one-fifth the value expected from the balance between its production rate by radioactive decay and its expected escape rate. This could indicate that there is some other means by which helium can escape the Earth, which might apply to other gases as well, and hence may be of great consequence to our understanding of atmospheric composition and evolution.

The Earth's magnetosphere (Fig. 2) is an even larger region of space containing magnetic fields, electric fields and energetic particles. It is formed by a complex and incompletely understood interaction between the Earth's magnetic field and the hot, supersonic ionized gas (the solar wind) streaming from the Sun. The magnetosphere extends some ten Earth radii $(64,000 \mathrm{~km}$, or 40,000 miles $)$ in the sunward direction and is drawn out, like the tail of a comet, to a distance of hundreds if not thousands of Earth radii in the anti-solar direction by the flow of the solar wind. Within this region complex electromagnetic processes go on which give rise to beautiful and spectacular auroral displays (Fig. 3), the Van Allen belts, and a host of interactions involving the magnetosphere, the Earth's ionosphere, and the neutral atmosphere. Space experimentation has revealed some of the processes which occur in the magnetosphere and has begun to clarify how these processes 


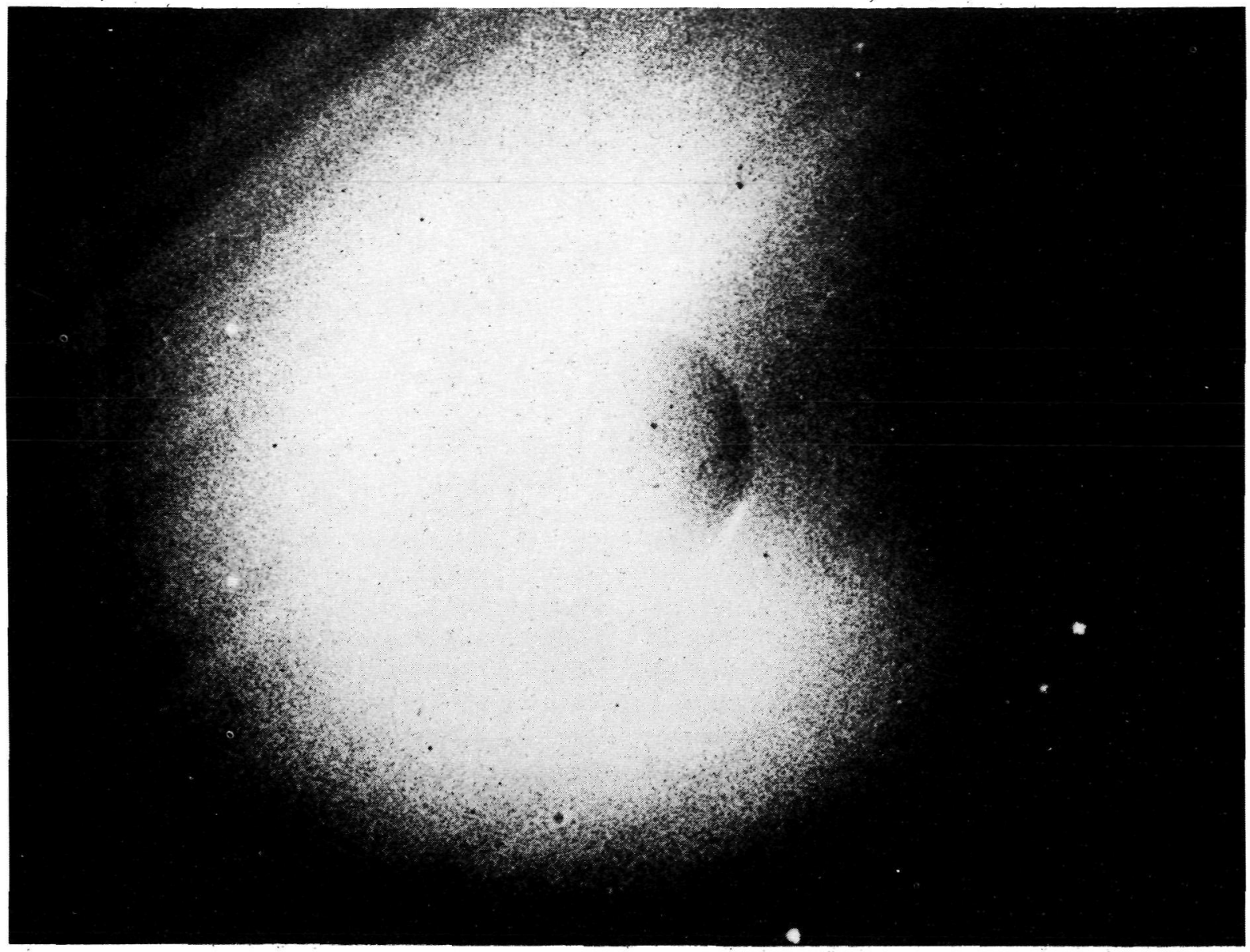

1 A far-ultraviolet photograph of the Earth, including the atomic hydrogen Lyman-alpha line at 121.6 nanometers. This picture was obtained by the Naval Research Laboratory Far Ultraviolet Camera from the lunar surface during the Apollo 16 mission. The diffuse glow is due to the hydrogen geocorona, which is detectable out to a distance of more than 15 Earth radii from the Earth. A portion of the southern polar auroral zone is visible on the lower right edge of the Earth. (Courtesy Naval Research Laboratory)

affect the environment in which we live and through which we communicate.

For a planet to have Van Allen belts, it must have a supply of particles to be trapped, and a magnetic field to trap them. The supply is, most likely, the solar wind. The Earth's magnetic field is believed to result from the circulation of liquid metal in the interior. The heat that melted the Earth's interior is also responsible for the building of continents, sea beds, and mountains, and for the distribution of mineral resources in the crust.

Within the framework of our current understanding, it appears that without its magnetic field, the composition of the Earth's atmosphere might be different. The magnetic field deflects the oncoming solar wind, preventing the bulk of it from coming in contact with our atmosphere. If the magnetic field were absent, the ionizing potential of the solar wind could interact directly with our atmosphere, possibly sweeping away some of it, and particularly its lighter components. It is even conceivable that the Earth's atmospheric water vapor might thereby have been depleted continuously to the point where life as we know it could no longer exist. Such a process may have occurred on Venus (see below).

Other planets are natural laboratories for the extension of the ranges and scales of phenomena also present on Earth. Exploration of the trapped radiation and magnetic fields of other planets, therefore, is an important tool in understanding the formation and development of all planets, including our own. This understanding is a matter not of intellectual interest alone, but bears on problems of locating scarce natural resources and controlling the long-term development of our atmosphere.

\section{MERCURY}

Our present knowledge of Mercury is relatively sparse, because it is the smallest planet and is so close to the Sun. It is believed to resemble the Moon in many respects. No atmosphere has been detected, and it shows markings similar to the dark 


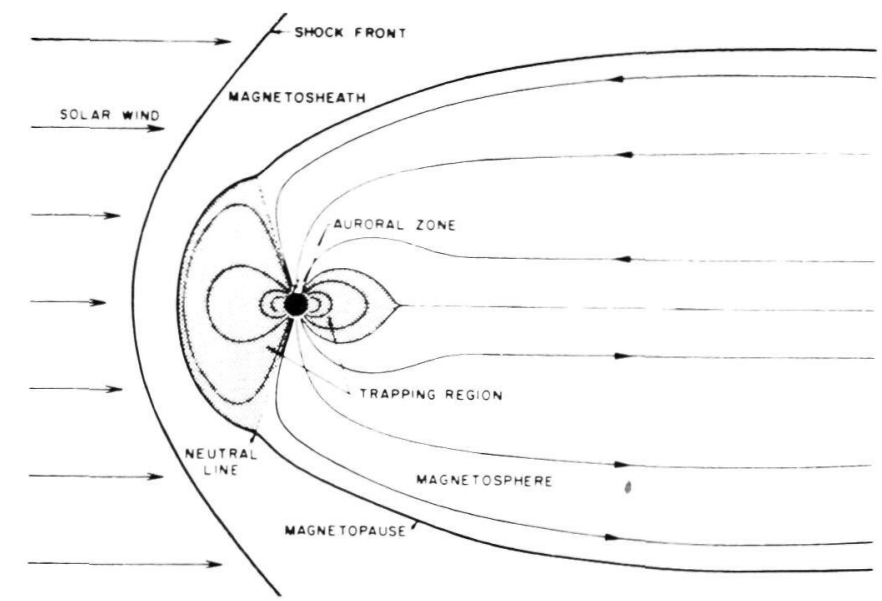

2 Structure of the terrestrial magnetosphere. The incoming solar wind is deflected by the Earth's magnetic field; the limit of penetration is known as the magnetopause. The deflection of the supersonic solar wind causes a shock front to occur up-sun of the magnetopause. Within the shaded regions of the magnetosphere, charged particles can be trapped by the Earth's magnetic field. At the borders of these trapping zones, particles can precipitate into the atmosphere, causing the polar aurorae. maria ("seas") of the Moon. Its day (sun) side gets very hot-more than $600 \mathrm{~K}\left(620^{\circ} \mathrm{F}\right)^{*}$ at local noon at the equator. On the night side, however, the temperature is very low (near $110 \mathrm{~K}$, or $-260^{\circ} \mathrm{F}$ ). As shown in Table 1, Mercury rotates on its axis very slowly, but it does not keep the same face always toward the Sun, as many astronomers thought until recently. Instead, it makes three rotations for every two revolutions about the Sun. Because of its small size and slow rotation, Mercury probably lacks an appreciable magnetic field and belts of trapped radiation. It may, however, have a molten iron core, in view of its high density and high surface temperature.

\section{VENUS}

Venus is the second planet outward from the Sun, and comes closer to the Earth than any other planet. It is also very similar to the Earth in size. Because it has a very dense atmosphere and is

*See footnote, Table 1
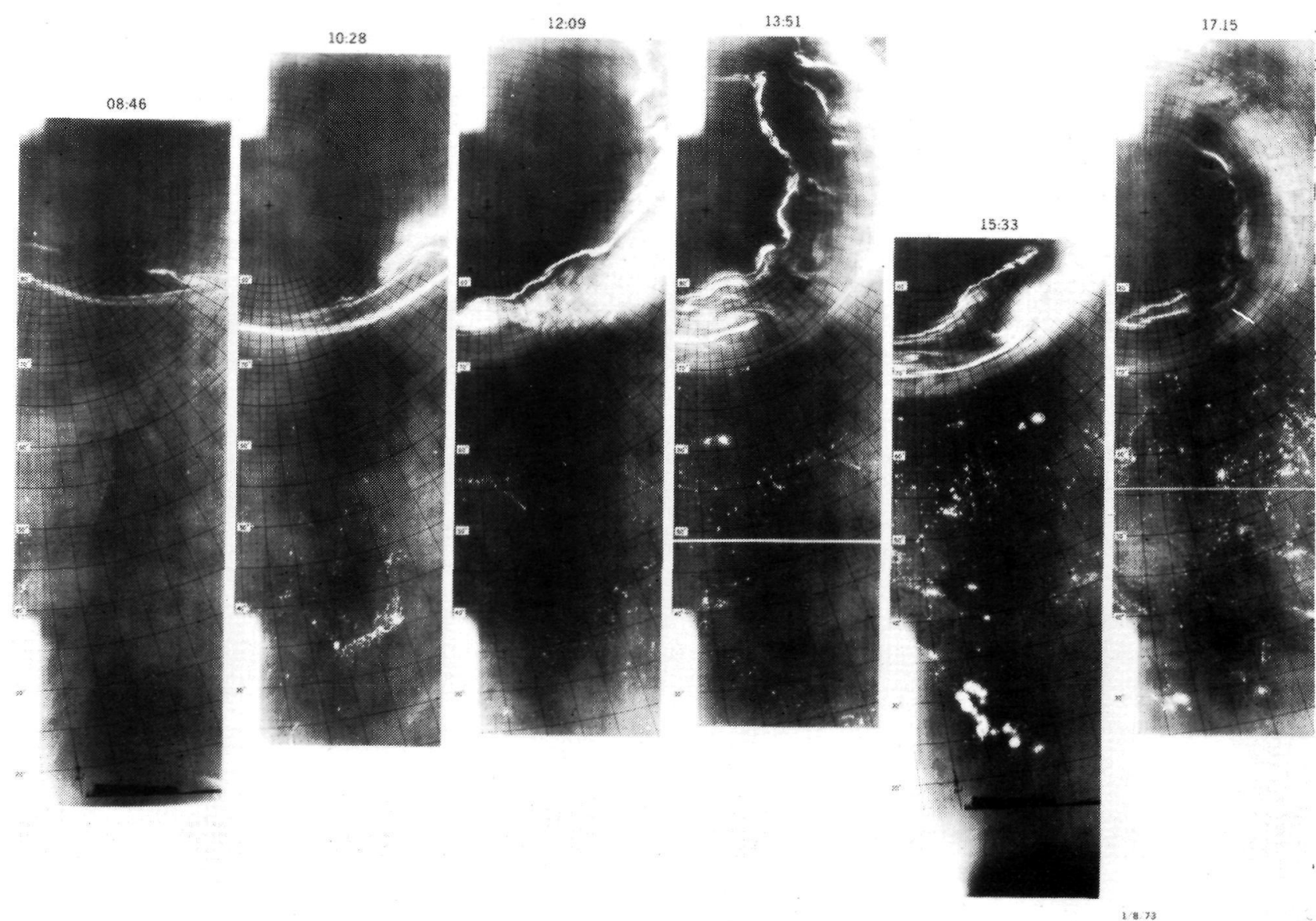

3 A sequence of data, obtained by a USAF meteorological satellite, showing the development of an auroral display over the northern polar regions. The figure is composed of six orbital passes, ordered from left to right. The lights of cities can be seen in the lower parts of each orbit of data. 


\begin{tabular}{|c|c|c|c|c|c|c|c|c|c|}
\hline & & OPERTIES OF $\mathrm{T}$ & THE PLANET & OF OUR $S$ & R SYSTEM & & & & \\
\hline & Mercury & venus & Earth & Mars & Jupiter & Saturn & Uranus & Neptune & Pluto \\
\hline Equatorial Radius $\left\{\begin{array}{l}(\mathrm{km}) * \\
(\text { in Earth radii }\end{array}\right.$ & i) $\begin{array}{l}2420 \\
0.38\end{array}$ & $\begin{array}{l}6100 \\
0.96\end{array}$ & $\begin{array}{l}6378 \\
1.00\end{array}$ & $\begin{array}{l}3380 \\
0.53\end{array}$ & $\begin{array}{l}71600 \\
11.19\end{array}$ & $\begin{array}{r}60400 \\
9.47\end{array}$ & $\begin{array}{r}25900 \\
3.73\end{array}$ & $\begin{array}{r}24750 \\
3.49\end{array}$ & $\begin{array}{l}\sim 3200 ? \\
\quad 0.52\end{array}$ \\
\hline Mass $($ Earth $=1)$ & 0.055 & 0.82 & 1.00 & 0.11 & 317.9 & 95.2 & 14.5 & 17.2 & $\sim 0.11 ?$ \\
\hline Density $\left(\mathrm{g} \mathrm{cm}^{-3}\right)($ water $=1)$ & 5.4 & 5.1 & 5.52 & 3.97 & 1.31 & 0.70 & 1.31 & 1.66 & $\sim 0.49 ?$ \\
\hline Surface Gravity $($ Earth $=1)$ & 1.38 & 0.89 & 1.00 & 0.38 & 2.6 & 1.2 & 0.96 & 1.5 & ? \\
\hline Escape Velocity $(\mathrm{km} / \mathrm{sec})$ & 4.2 & 10.3 & 11.2 & 5.0 & 60 & 36 & 21 & 24 & $\sim 5.3 ?$ \\
\hline Orbital Velocity $(\mathrm{km} / \mathrm{sec})$ & 48 & 35 & 30 & 24 & 13 & 10 & 7 & 5 & 5 \\
\hline Rotation Period** & $59^{d}$ & $\begin{array}{l}243^{\mathrm{d}} \\
\text { (retrograde) }\end{array}$ & $23^{\mathrm{h}} 56^{\mathrm{m}}$ & $24^{\mathrm{h}} 37^{\mathrm{m}}$ & $9^{\mathrm{h}} 55^{\mathrm{m}}$ & $10^{\mathrm{h}} 24^{\mathrm{m}}$ & $\begin{array}{l}10^{\mathrm{h}} 50^{\mathrm{m}} \\
\text { (retrograde }\end{array}$ & $15^{\mathrm{h}} 40^{\mathrm{m}}$ & $6^{\mathrm{d}} \cdot 25$ \\
\hline $\begin{array}{l}\text { Inclination of Equator } \\
\text { to Orbit }\end{array}$ & $30^{\circ}$ & $177^{\circ}$ & $23^{\circ} .5$ & $24^{\circ} .0$ & $3^{\circ} .1$ & $26^{\circ} .7$ & $97^{\circ} .9$ & $28^{\circ} .8$ & ? \\
\hline $\begin{array}{l}\text { Albedo (ratio: reflected to } \\
\text { incident sunlight) }\end{array}$ & 0.06 & 0.49 & 0.37 & 0.14 & 0.45 & 0.61 & 0.35 & 0.35 & $0.15 ?$ \\
\hline Number of Known Satellites & 0 & 0 & 1 & 2 & 12 & 10 & 5 & 2 & 0 \\
\hline Surface Temp. (K)** & $110-620$ & 700 & $\begin{array}{l}287 \\
\text { (avg) }\end{array}$ & $\begin{array}{l}145-300 \\
(248 \text { avg })\end{array}$ & 135 & 97 & 55 & $\sim 45$ & ? \\
\hline Lowest Visible Surface & solid & cloud & solid & solid & cloud & cloud & cloud & cloud & ? \\
\hline Period of Revolution ** & $87^{d} \cdot 97$ & $224^{\mathrm{d}} \cdot 70$ & $365^{d} \cdot 26$ & $686^{\mathrm{d}} .98$ & $11^{\mathrm{y}} \cdot 86$ & $29^{\mathrm{y}} .46$ & $84^{\mathrm{y}} \cdot 01$ & $164^{\mathrm{y}} .79$ & $248^{\mathrm{y}} .4$ \\
\hline 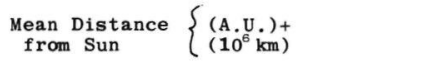 & $\begin{array}{l}0.387 \\
57.90\end{array}$ & $\begin{array}{r}0.723 \\
108.16\end{array}$ & $\begin{array}{c}1.0 \\
149.60\end{array}$ & $\begin{array}{l}1.524 \\
227.99\end{array}$ & $\begin{aligned} 5.203 \\
778.37\end{aligned}$ & $\begin{array}{l}9.539 \\
1427.0\end{array}$ & $2869.3 \quad 4$ & $\begin{array}{r}30.07 \\
4497.0\end{array}$ & $\begin{array}{c}39.44 \\
5900.2\end{array}$ \\
\hline Eccentricity of Orbit & 0.2056 & 0.0068 & 0.0168 & 0.0934 & 0.0485 & 0.0556 & 0.0472 & 0.0086 & 0.2494 \\
\hline Inclination of Orbit to Ecliptic & $7^{\circ} 00^{\prime}$ & $3^{\circ} 24$ & 0 & $1^{\circ} 51$ & $1^{\circ} 18$ & $2^{\circ} 29^{\prime}$ & $0^{\circ} 46^{\prime}$ & $1^{\circ} 46^{\prime}$ & $17^{\circ} 12^{\prime}$ \\
\hline $\begin{aligned} & * \quad 1 \mathrm{~km}=0.62 \mathrm{mile} \\
&+\quad 1 \text { Astronomical Unit }=149.6 \mathrm{x} \\
&=\text { mean di }\end{aligned}$ & $\begin{array}{l}\mathrm{km} \\
\mathrm{ce} \text { of }\end{array}$ & th from $S$ & ** & $\begin{array}{l}\text { The Kelvi } \\
\text { scale usir } \\
700 \mathrm{~K}=8\end{array}$ & $\begin{array}{l}\text { perat } \\
\text { ntigr } \\
287\end{array}$ & $\begin{array}{l}\text { cale } \\
\text { egre } \\
57^{c}\end{array}$ & $\begin{array}{l}\text { absolute } \\
100 \mathrm{~K}=\end{array}$ & $\begin{aligned} \text { temp } \\
\text { xampl } \\
-280\end{aligned}$ & \\
\hline
\end{tabular}

$\overline{* * y=y e a r ; d=\text { day }} \quad h=$ hour $; m=$ minute

completely covered by clouds (see Fig. 4), we have no visual knowledge of its surface features. Its atmosphere is almost pure carbon dioxide $\left(\mathrm{CO}_{2}\right)$. Its surface pressure is 100 bars (100 times that of atmospheric pressure on the Earth), and its surface temperature is a scorching $750 \mathrm{~K}\left(900^{\circ} \mathrm{F}\right)$. Although carbon dioxide is abundant in the Venus atmosphere per ground-based optical observations, these measurements reached only to the top of the Venus cloud layer, about $60 \mathrm{~km}$ ( 37 miles) above the surface, where the pressure is a fraction of $1 \mathrm{bar}$ and the temperature is 220 to $250 \mathrm{~K}$. Near the base of the cloud layer, the pressure is about $1 \mathrm{bar}$ and the temperature is a livable $295 \mathrm{~K}$ $\left(70^{\circ}\right)$. The earliest indication of the surprising high surface temperature, however, was given by ground-based microwave radio observations of Venus. These findings were later confirmed by close-up measurements during the Mariner 5 fly-by, and by several Russian probes which entered the atmosphere of Venus (some reaching the surface), measuring the pressure and composition of the atmosphere as well as the temperature. Fig. 5 shows the variations of temperatures and pressure with altitude above the surface of Venus, and the probable cloud layers, based on American and Soviet data.

The extreme temperature of the surface of Venus is believed explicable by the so-called "greenhouse effect" in the dense $\mathrm{CO}_{2}$ atmosphere. Incoming so- lar radiation filters through the atmosphere and heats the surface. Then, the heat is "trapped" and prevented from radiating away, by the insulating property of the dense atmosphere.

The most surprising fact about the composition of Venus' atmosphere is the almost complete lack of water. The water in the Earth's oceans would be equivalent to an atmospheric pressure of 300 bars if it were completely vaporized. The amount of water vapor on Venus, however, is less than 1 atmosphere equivalent; i.e., less than $1 / 300$ of the total amount of water on Earth.

The Earth's atmosphere contains only a very small proportion of carbon dioxide, but a large quantity of $\mathrm{CO}_{2}$ is present in the Earth's crust in the form of carbonate rocks (such as limestone, or calcium carbonate). This amount of $\mathrm{CO}_{2}$ would produce an atmospheric pressure of more than 70 bars if released, and is, therefore, almost equal to the amount found in the atmosphere of Venus. The relative amounts of $\mathrm{H}_{2} \mathrm{O}, \mathrm{CO}_{2}$, and $\mathrm{N}_{2}$ on Earth are about the same as their relative proportions in volcanic gases. The reason that the bulk of the Earth's $\mathrm{CO}_{2}$ is in carbonate rocks, rather than in the atmosphere, as on Venus, is that carbonate rocks are formed by reaction of silicate rocks with $\mathrm{CO}_{2}$ only in the presence of liquid water. Since Venus has probably always been too hot for water to exist in liquid form, this would explain why the $\mathrm{CO}_{2}$ has remained in the atmosphere. However, 
4 A composite ultraviolet-light photograph of Venus taken by Mariner 10 on February 6, 1974. The cloud patterns show the general circulation of the upper atmosphere. The south ecliptic pole is in the bottom frame, and the morning terminator is at the right. (Courtesy NASA)

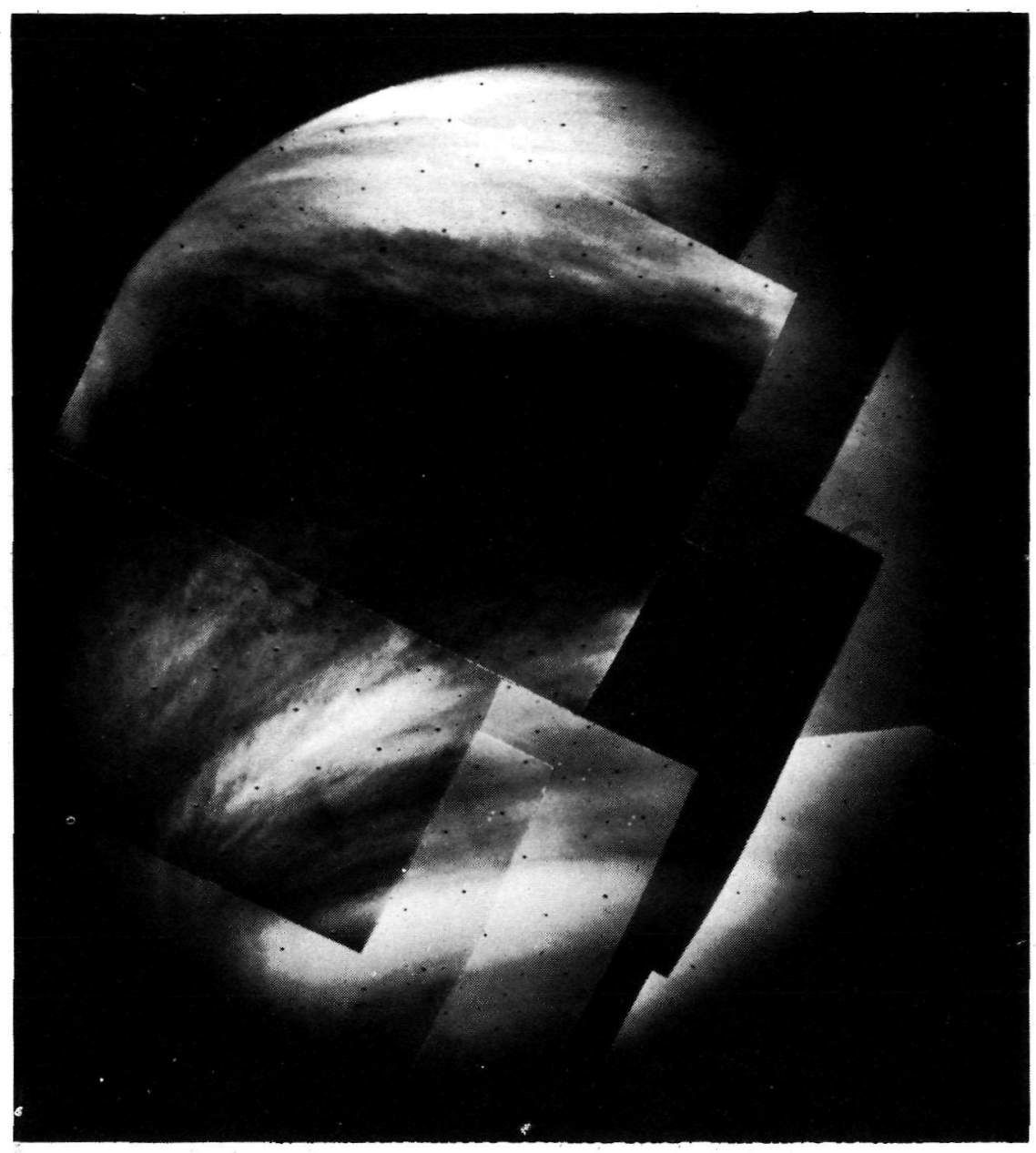

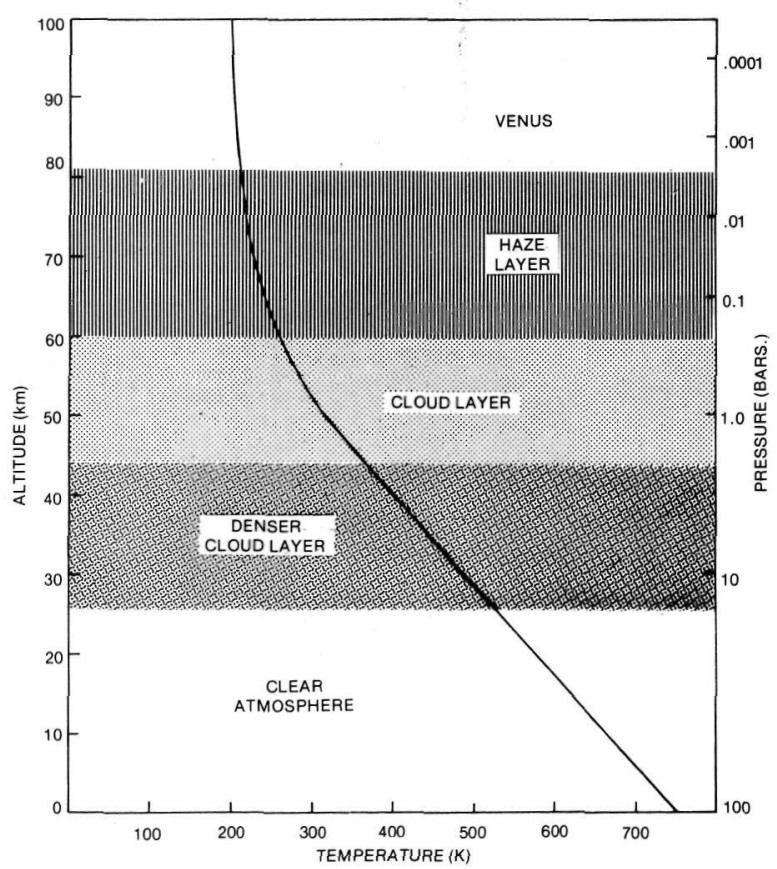

5 Variation of temperature with altitude in the Venus atmosphere, based on a combination of ground-based observations and probe measurements. On the right are shown the pressures corresponding to the different altitudes. the puzzling question is, what happened to the water vapor? Perhaps Venus formed with less water originally than did the Earth, as a result of its closer proximity to the primitive Sun, or perhaps somehow the water has escaped over the 4.5 billion years the solar system has existed. It is believed by some that the water may have been lost from Venus as $\boldsymbol{x}$ result of "sweeping" of hydrogen (produced by photodissociation of water vapor) by the solar wind, which approaches the planet very closely due to its lack of an appreciable magnetic field (see Fig. 6). Such a process may have operated at times on the Earth during periods of magnetic field reversals (there is geological evidence for such reversals at perhaps 70,000-year intervals). The oxygen left behind would remain in the Earth's atmosphere, but on Venus it probably exists only in the form of oxides of surface materials. Only much more complete measurements will shed some light on why the atmospheres of Venus and Earth have evolved so divergently.

The composition of the clouds of Venus is also far from known. We know that they are not composed of water droplets or ice crystals, as are ter- 
restrial clouds, both from ground-based and spaceprobe measurements. Suggestions for the cloud composition have included carbon dioxide, saturated hydrochloric acid, sulfuric acid, and many others; very recent observations have identified sulfuric acid. If the sulfuric acid is in aqueous solution, this may account in part for the scarcity of water on Venus.

The surface features of Venus are known at present only by radar measurements from Earth. These have indicated possible mountain ranges and other topographic features. It is likely that only radar imagery from a Venus orbiter can provide a high-resolution map, since even probes which descend to the surface can sample only a very limited area.

Venus rotates on its axis very slowly-once every 243 days, which is longer than the Venus year of 225 days. Space probes so far have not detected any magnetic field comparable to the Earth's, nor anything equivalent to the Earth's Van Allen belts.

\section{MARS}

Mars (Fig. 7) is the fourth planet outward from the Sun; i.e., the next one beyond the Earth's orbit. Its diameter is about half that of the Earth. Because of its nearness (it can approach almost as close as can Venus) and its clear atmosphere, its surface can be studied in greater detail than any other planet with ground-based telescopes, and therefore more is known about Mars than any other planiet.

As in the case of Venus, Mars' atmosphere is almost pure carbon dioxide. However, the atmospheric pressure is less than $1 / 100$ that of the Earth's, and the temperatures range from $145 \mathrm{~K}$ $\left(-200^{\circ} \mathrm{F}\right)$ to $300 \mathrm{~K}\left(80^{\circ} \mathrm{F}\right)$. There is no appreciable amount of liquid water on Mars, due not only to its low average temperature but also to its very low atmospheric pressure. At pressures less than 6.1 millibars (.09 pounds per square inch), only gaseous and solid phases of water can exist (as is true of carbon dioxide at Earth's atmospheric pressure). Only limited areas of the Martian surface have atmospheric pressures greater than 6.1 millibars. There is, however, evidence for water in the form of ice in the polar caps, in hydrated minerals; and possibly underground as permafrost.

Also, as in the case of Venus, carbon dioxide had been detected in the Martian atmosphere many years ago, but it was not until the first measurements with Mariner spacecraft that it was shown that $\mathrm{CO}_{2}$ makes up most of the total atmosphere. For example, ultraviolet spectroscopy on the Mariner 6 and 7 spacecraft showed that less than one percent of nitrogen was present in the at-

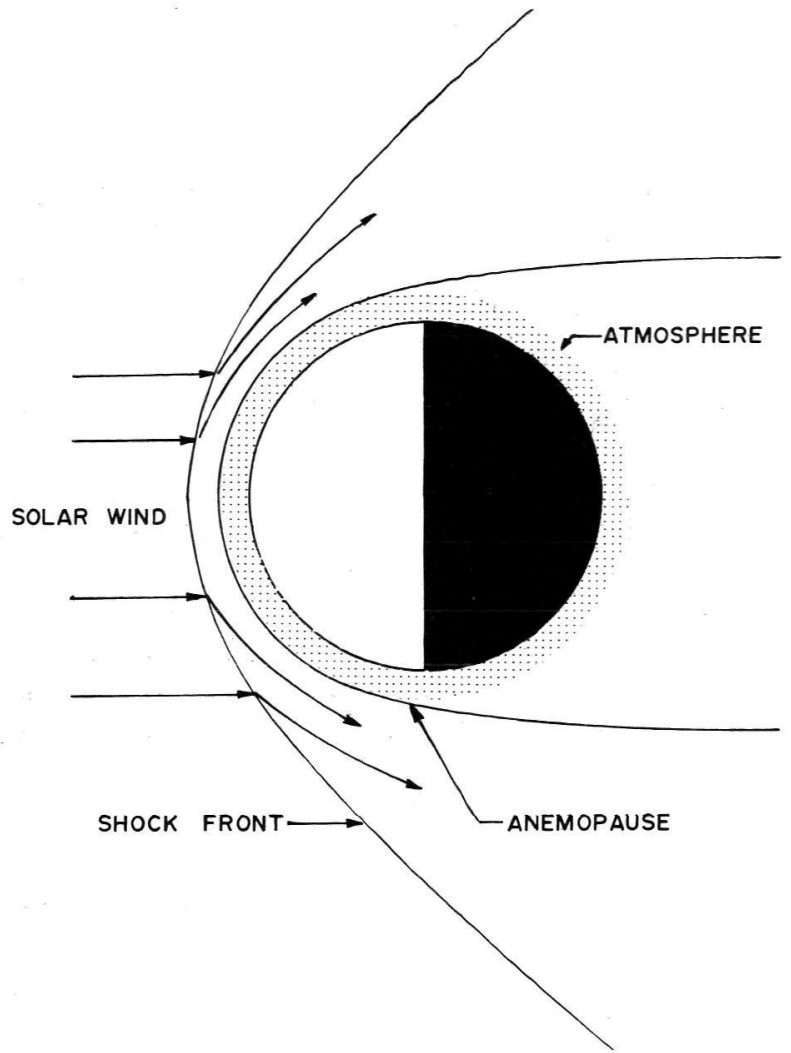

6 For a planet with no appreciable magnetic field, the solar wind can approach much closer to the planet-that is, to the point at which the solar wind pressure equals the atmospheric pressure (anemopause). Any ionized atmospheric constituents which cross upward across the anemopause boundary can be swept away by interactions with the solar wind.
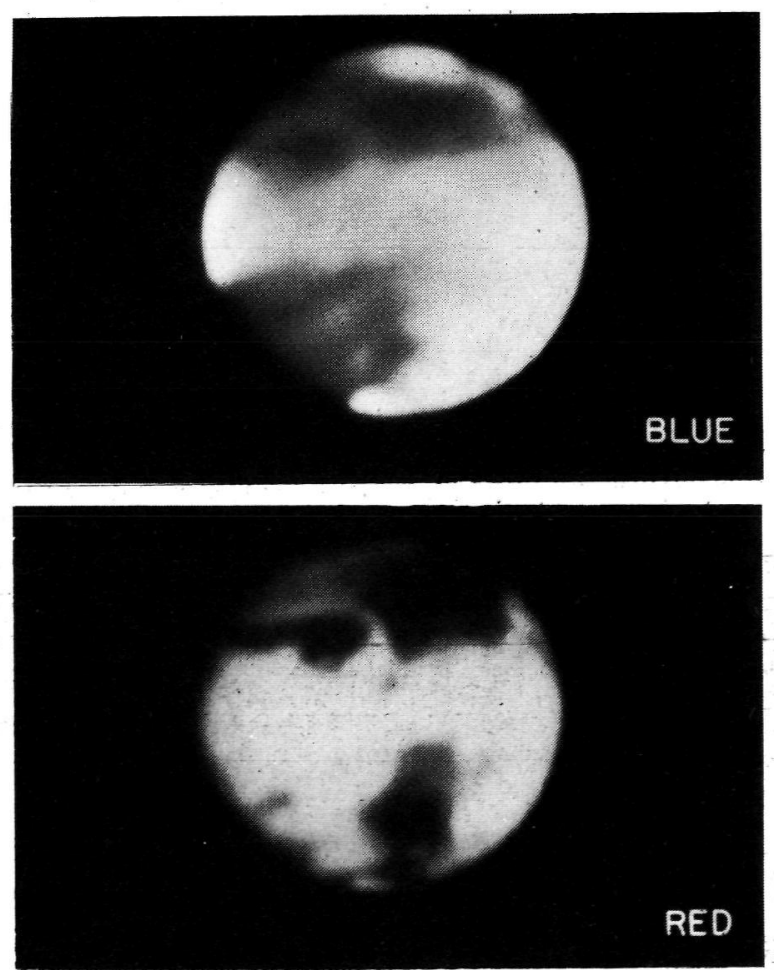

7 Photographs of Mars obtained with the 200-inch Palomar telescope. Note the lower contrast of surface features in blue light compared to red light. (Courtesy The Hale Observatories) 
mosphere. (Nitrogen and the inert gases helium, argon, etc., cannot be detected in planetary atmospheres by ground-based observations.) Other measurements showed that the observed amount of carbon dioxide was essentially equal to the observed total atmosphere-for example, radio occultation measurements (as the spacecraft passed behind Mars), which gave the total atmospheric pressure, agreed with infrared spectrometric measurements of the $\mathrm{CO}_{2}$ abundance. The polar caps of Mars are composed partly and perhaps mainly of frozen $\mathrm{CO}_{2}$ (dry ice), but water ice is also present. Radiometric measurements from Mariner 9 showed the interiors of the polar caps in winter to be at temperatures consistent with those where $\mathrm{CO}_{2}$ could vaporize at the Martian atmospheric pressure-about $148 \mathrm{~K}\left(-194^{\circ} \mathrm{F}\right)$.

The Mariner 9 instruments also indicated that there is water vapor in the atmosphere of Mars, but much less than in the Earth's atmosphere. Although there had been indications of its presence in Earth-based observations, it was difficult to detect. (A major difficulty in ground-based observations of water in the atmospheres of other plan- ets is the problem of separating out the effects of the much more abundant water in our own atmosphere.)

Atomic hydrogen has also been detected in the Martian upper atmosphere, forming an extended corona similar to the terrestrial hydrogen geocorona which was shown in Fig. 1. Since hydrogen is a very light gas and could not possibly be retained as a permanent atmospheric constituent by the weak gravity of Mars, it could only be there as a result of the breakup of water molecules by solar ultraviolet radiation (photodissociation). This process is also responsible for the hydrogen in the Earth's outermost atmosphere. In the case of Mars, the loss rate of water by this process is approximately 100,000 gallons per day. This amounts to a layer of water 4 meters (13 feet) thick, covering the entire planet, over geological time. Photodissociation of water vapor and carbon monoxide also produces small amounts of atomic oxygen and carbon monoxide in the Martian atmosphere, which have also been detected.

Mars' surface, as observed by the television cameras aboard the Mariner fly-bys and the Mariner
8 Nix Olympica, a gigantic volcanic mountain on Mars, as photographed by the Mariner 9 orbiter in January 1972. The mountain is more than 500 kilometers $(310$ miles) across at its base, more than twice as broad as the la rgest volcanic pile on Earth (the island of Hawaii). Also its height, about $23 \mathrm{~km}$ (70,000 feet) above the surrounding plain, is more than twice that of Mt. Everest. (Courtesy NASA)

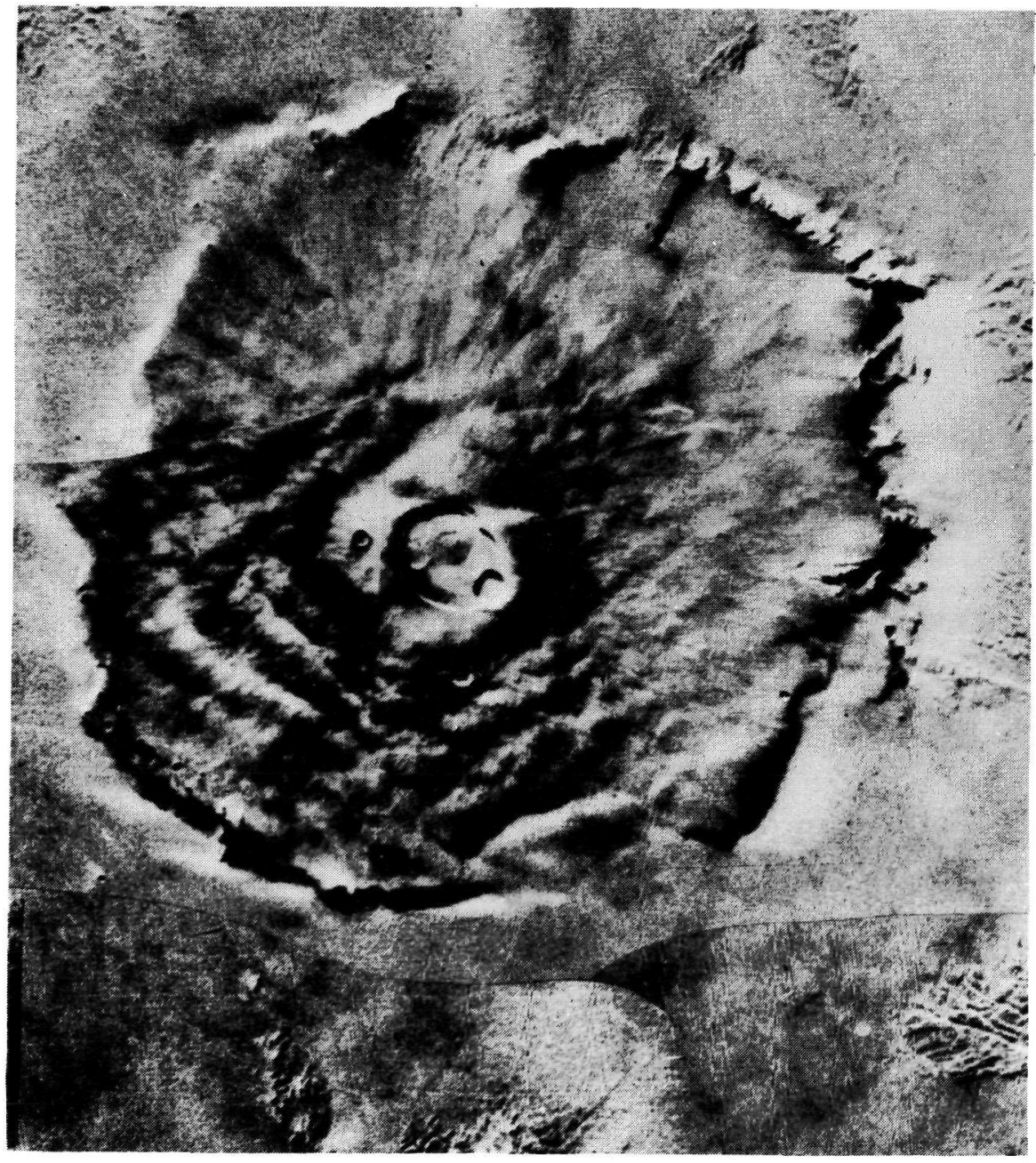




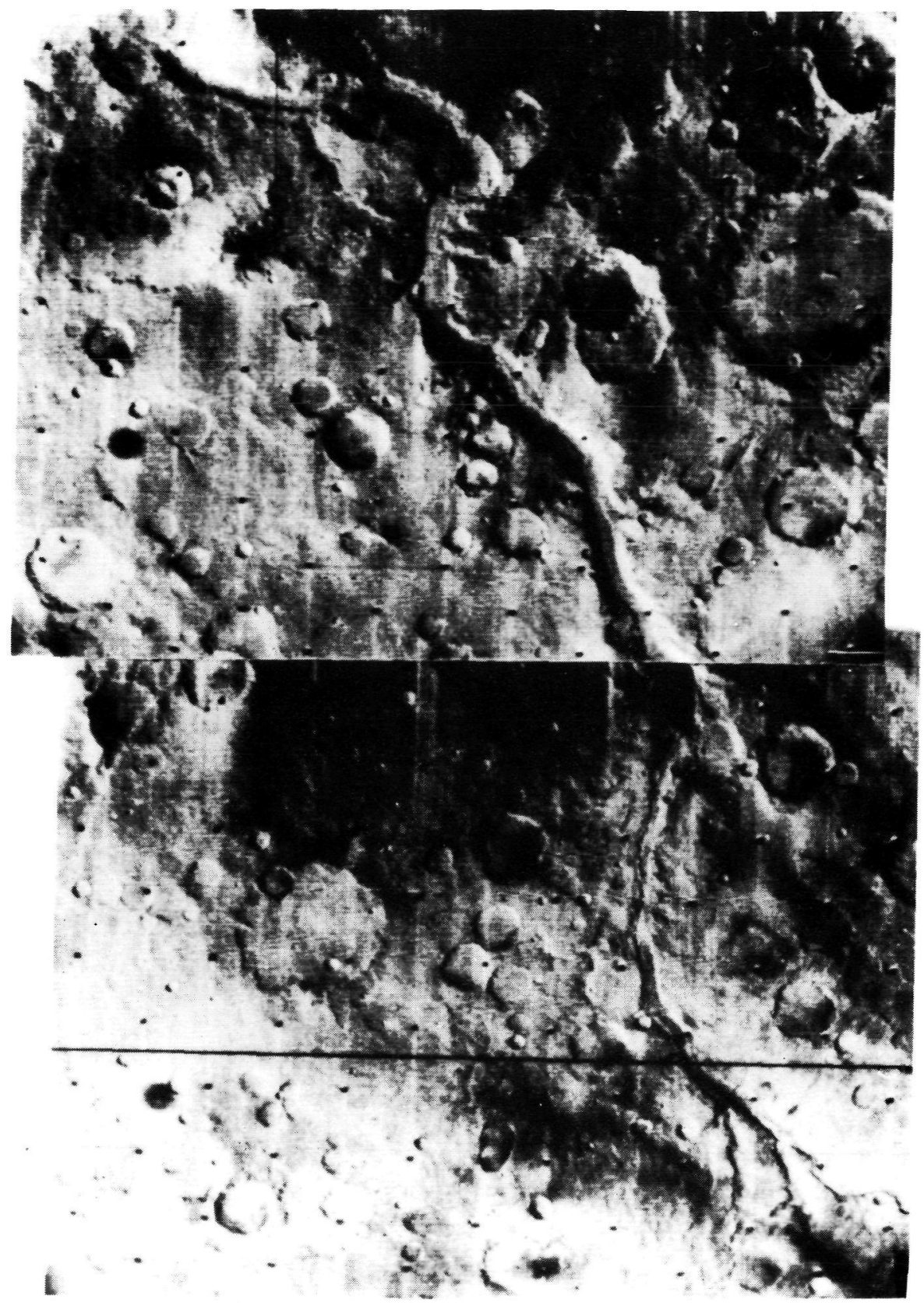

9 This 700-kilometer (440-mile) long sinuous valley, photographed by the Mariner 9 Mars orbiter, appears to have been eroded by some sort of flowing fluidindicating that perhaps liquid water existed on Mars in the past. (Courtesy NASA)
9 orbiter, show many impact craters, like the Moon's, presumably produced by large meteorites early in the history of the solar system. However, there is evidence for far more in the way of erosional processes and geological activity than on the Moon. Some areas of Mars are free of craters and hence may be geologically quite young. Gigantic extinct volcanoes, such as Nix Olympica (Fig. 8), and geological fault zones, far larger than any observed even on the Earth, are present. Tremendous dust storms frequently sweep the Martian surface; the one in late 1971 completely hid the surface of the planet for weeks after the arrival of Mariner 9. Perhaps the most interesting finding is the presence of what appear to be dry river beds (Fig. 9)
- evidence that at some time in its history, liquid water probably existed on Mars.

Mariner 9 also provided the first close-up pictures (Fig. 10) of Mars' two tiny satellites, Phobos and Deimos (see Table 2). Phobos, the larger of the two, is a chunk of rock $20 \mathrm{~km}$ (12 miles) long and $10 \mathrm{~km}$ (6 miles) across. Deimos is about half the size of Phobos. The latter orbits Mars only some $6000 \mathrm{~km}$ (3700 miles) above its surface, and completes an orbit around Mars in less time than Mars takes to rotate on its axis. Hence, it appears to an observer on the Martian surface to rise in the west and set in the east. Phobos and Deimos appear to be made of very dark material, reflecting only 6 percent of the sunlight incident on them. 


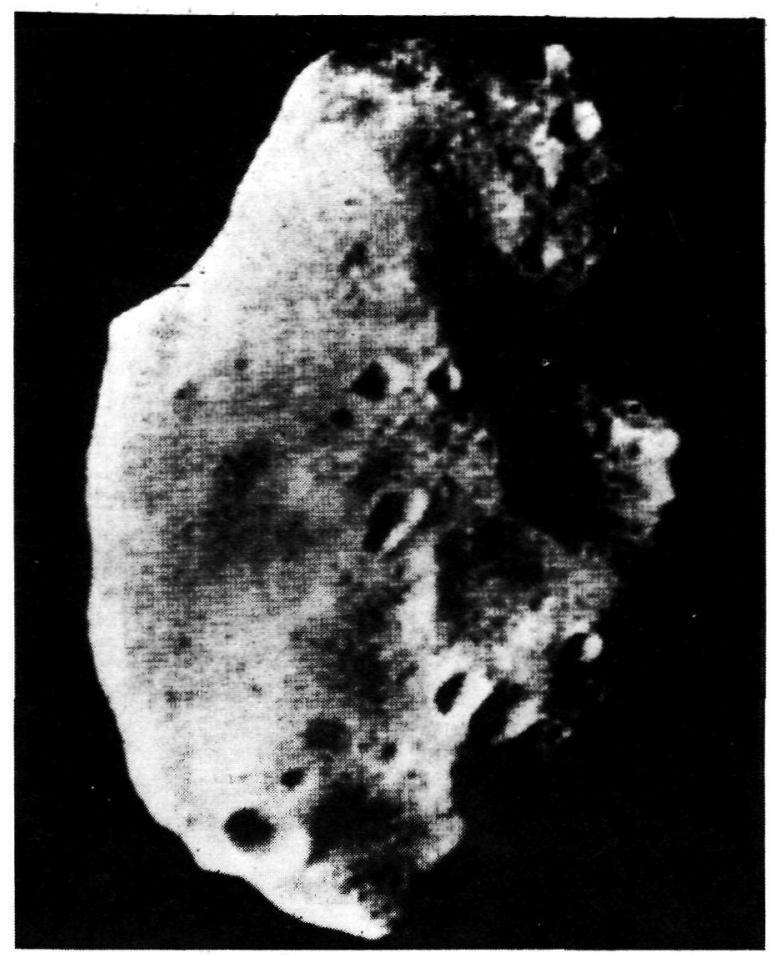

10 A closeup view of Phobos, the larger and closer of the two Martian satellites, obtained by Mariner 9 . (Courtesy NASA)
Like Venus, Mars has only a very weak magnetic field and no zones of trapped radiation. Recent Soviet data confirm earlier estimates that its field is about 2/10,000 that of Earth's. This suggests that Mars lacks a molten core, at least one large enough to sustain the circulation needed to produce a substantial magnetic field. If Mars' interior has never melted, the distributions of minerals in its crust may be quite different from that on Earth. The indication that liquid water may have existed on Mars previously suggests that it may at one time have had a significant magnetic field.

\section{ASTEROIDS}

Most of the asteroids, or minor planets, lie in the region between the orbits of Mars and Jupiter. To date more than 2000 of the minor planets are known by their orbits. There are probably tens of thousands of of them large enough to be observed with existing telescopes.

Ceres, the largest, has a diameter of about 770 $\mathrm{km}$ (480 miles). Only a few hundred are over $40 \mathrm{~km}$ ( 25 miles) across, and most are less than $5 \mathrm{~km}$ in diameter. None is large enough to have a detect-

TABLE 2

\begin{tabular}{|c|c|c|c|c|c|c|c|}
\hline \multirow[b]{2}{*}{ Planet } & \multirow[b]{2}{*}{ Satellite } & \multirow[b]{2}{*}{$\begin{array}{c}\text { Mean Distance } \\
\text { from Planet, } \\
\text { km } \\
\end{array}$} & \multicolumn{3}{|c|}{ SATELLITES OF THE SOLAR SYSTEM } & \multicolumn{2}{|c|}{ Mass Ratio } \\
\hline & & & $\begin{array}{l}\text { Sidereal } \\
\text { Period of } \\
\text { Revolution }\end{array}$ & $\underset{\mathrm{km}}{\mathrm{Radius},}$ & $\begin{array}{l}\text { Radius/ } \\
\text { Moon's/Radius }\end{array}$ & $\begin{array}{r}\text { Mass } \\
\text { Satellite/planet }\end{array}$ & $\frac{\text { Ratio }}{\text { Satellite/Moon }}$ \\
\hline Earth & Moon & 384,402 & $27^{\mathrm{d}} 7^{\mathrm{h}} 43^{\mathrm{m}}$ & 1,738 & 1.0 & 0.0123 & 1.0 \\
\hline \multirow[t]{2}{*}{ Mars } & Phobos & 9,350 & $0^{d_{7} h_{39}} 9^{m}$ & 8 & $4.6 \times 10^{-3}$ & & \\
\hline & Deimos & 23,500 & $1^{\mathrm{d}} 6^{\mathrm{h}} 17^{\mathrm{m}}$ & 4 & $2.3 \times 10^{-3}$ & & \\
\hline \multirow[t]{12}{*}{ Jupiter } & v & 181,500 & $0^{d_{11}} h_{57^{m}}$ & 100 & 0.057 & & \\
\hline & Io & 422,000 & $1^{\mathrm{d}} 18^{\mathrm{h}} 27^{\mathrm{m}}$ & 1,829 & 1.05 & $3.8 \times 10^{-5}$ & 0.985 \\
\hline & Europa & 671,400 & $3^{\mathrm{d}} 13^{\mathrm{h}} 14^{\mathrm{m}}$ & 1,550 & 0.89 & $2.5 \times 10^{-5}$ & 0.641 \\
\hline & Ganymede & $1,071,000$ & $7^{\mathrm{d}} 3^{\mathrm{h}} 43^{\mathrm{m}}$ & 2,775 & 1.60 & $8.2 \times 10^{-5}$ & 2.112 \\
\hline & Callisto & $1,884,000$ & $16^{\mathrm{d}} 16^{\mathrm{h}} 32^{\mathrm{m}}$ & 2,500 & 1.44 & $5.1 \times 10^{-5}$ & 1.316 \\
\hline & vI & $11,500,000$ & $250^{\mathrm{d}}$ & 60 & 0.034 & & \\
\hline & VII & $11,750,000$ & $260^{d}$ & 20 & 0.011 & & \\
\hline & $\mathrm{x}$ & $11,750,000$ & $260^{\mathrm{d}}$ & 10 & $5.75 \times 10^{-3}$ & & \\
\hline & XII & $21,000,000$ & $625^{\mathrm{d}}$ & 10 & $5.75 \times 10^{-3}$ & & \\
\hline & XI & $22,500,000$ & $692^{d}$ & 12 & $6.90 \times 10^{-3}$ & & \\
\hline & VIII & $23,500,000$ & $739^{d}$ & 20 & 0.011 & & \\
\hline & IX & $23,700,000$ & $758^{d}$ & 11 & $6.33 \times 10^{-3}$ & & \\
\hline \multirow[t]{10}{*}{ Saturn } & Janus & 168,700 & $0^{\mathrm{d}} 19^{\mathrm{h}} 33^{\mathrm{m}}$ & & & & \\
\hline & Mimas & 185,700 & $0^{\mathrm{d}} 22^{\mathrm{h}} 37^{\mathrm{m}}$ & 230 & 0.13 & $6.7 \times 10^{-12}$ & 0.000052 \\
\hline & Enceladus & 238,200 & $1^{d} 8^{h_{5}} 3^{m}$ & 275 & 0.16 & $1.5 \times 10^{-7}$ & 0.001 \\
\hline & Tethys & 294,800 & ${ }_{1}^{\mathrm{d}} 21^{\mathrm{h}} 18^{\mathrm{m}}$ & 600 & 0.35 & $1.1 \times 10^{-6}$ & 0.0088 \\
\hline & Dione & 377,700 & $2^{\mathrm{d}} 17^{\mathrm{h}} 41^{\mathrm{m}}$ & 410 & 0.24 & $1.8 \times 10^{-6}$ & 0.0143 \\
\hline & Rhea & 527,500 & $4^{\mathrm{d}} 12^{\mathrm{h}} 25^{\mathrm{m}}$ & 650 & 0.37 & $3.0 \times 10^{-6}$ & 0.02 \\
\hline & Titan & $1,223,000$ & $15^{\mathrm{d}_{22}} \mathrm{~h}_{41^{\mathrm{m}}}$ & 2,425 & 1.40 & $2.5 \times 10^{-4}$ & 1.87 \\
\hline & Hyperion & $1,484,000$ & $22^{\mathrm{d}} 6^{\mathrm{h}_{3}} 8^{\mathrm{m}}$ & 200 & 0.115 & & \\
\hline & I apetus & $3,563,000$ & $79^{d} 7^{h_{56}}{ }^{m}$ & 650 & 0.37 & $2.5 \times 10^{-6}$ & 0.02 \\
\hline & Phoebe & $12,950,000$ & $551^{d}{ }^{h}$ & 140 & 0.080 & & \\
\hline \multirow[t]{5}{*}{ Uranus } & Miranda & 130,100 & ${ }_{1} \mathrm{~d}_{24}{ }^{\mathrm{m}} 50^{\mathrm{s}}$ & & & & \\
\hline & Ariel & 191,800 & $2^{\mathrm{d}} 12^{\mathrm{m}} 29^{\mathrm{s}}$ & 300 & 0.173 & & \\
\hline & Umbriel & 267,300 & $4^{\mathrm{d}} 3^{\mathrm{m}} 28^{\mathrm{s}}$ & 200 & 0.115 & & \\
\hline & Titania & 438,700 & $8^{\mathrm{d}} 16^{\mathrm{m}}{ }_{56^{\mathrm{S}}}^{\mathrm{s}}$ & 500 & 0.288 & & \\
\hline & Oberon & 586,600 & $13^{\mathrm{d}} 11^{\mathrm{m}} 7^{\mathrm{s}}$ & 400 & 0.230 & & \\
\hline \multirow[t]{2}{*}{ Neptune } & Triton & 353,600 & $5^{\mathrm{d}} 21^{\mathrm{h}} 3^{\mathrm{m}}$ & 1,885 & 1.08 & $1.3 \times 10^{-3}$ & 1.85 \\
\hline & Nereid & $6,000,000$ & $500^{d}$ & 150 & 0.086 & & \\
\hline
\end{tabular}


able atmosphere. The total mass of the asteroids is less than a thousandth of the Earth's mass. Hence, it is unlikely that the asteroids resulted from the break-up in the distant past of a planet orbiting between Mars and Jupiter. It is more likely that the matter in the asteroid belt represents a state of incomplete accumulation of the matter of which planets are formed.

A few of the asteroids move in orbits closer to the Sun than the Earth or even Mercury. Some, the so-called "Trojan" asteroids, are locked in the Jupiter orbit at positions $60^{\circ}$ preceding and following Jupiter on its motion around the Sun. Possibly these asteroids are remnants of the dusty and icy components of the original Jovian "jet stream" (in the primordial solar-system gas cloud) that aggregated into the planet.

Recent discoveries of asteroidal orbits that are bound in resonance with orbits of the Earth and other planets have fostered the speculation that the asteroids have existed along with the planets throughout a major span of the history of the solar system.

It is believed by some that most of the asteroids are made up of silicate rocks, nickel-iron, and other earthy materials and that most meteorites which strike the Earth are produced by collisions between asteroids, because optical tracking data on the paths of these meteorites through the Earth's atmosphere indicate that they traveled in orbits having their farthest point within the asteroid belt. Carbonaceous chondrites, a form of meteorite containing hydrocarbon compounds, indicate that such material is also present in the asteroid belt.

\section{JUPITER}

"Even from twenty million miles away, Jupiter was already the most conspicuous object in the sky ahead. The planet was now a plae, salmon-hued disk, about half the size of the Moon as seen from Earth, with the dark, parallel bands of its cloud belts clearly visible. Shuttling back and forth in the equatorial plane were the brilliant stars of Io, Europa, Ganymede, and Callisto-worlds that elsewhere would have counted as planets in their own right, but which here were merely satellites of a giant master.

"Through the telescope, Jupiter was a glorious sight-a mottled, multicolored globe that seemed to fill the sky. It was impossible to grasp its real size; Bowman kept reminding himself that it was eleven times the diameter of Earth, but for a long time this was a statistic with no real meaning.

"The telescopic cameras were operating contantly as the ship cut across the orbit of the giant inner satellites-every one of them as large as the

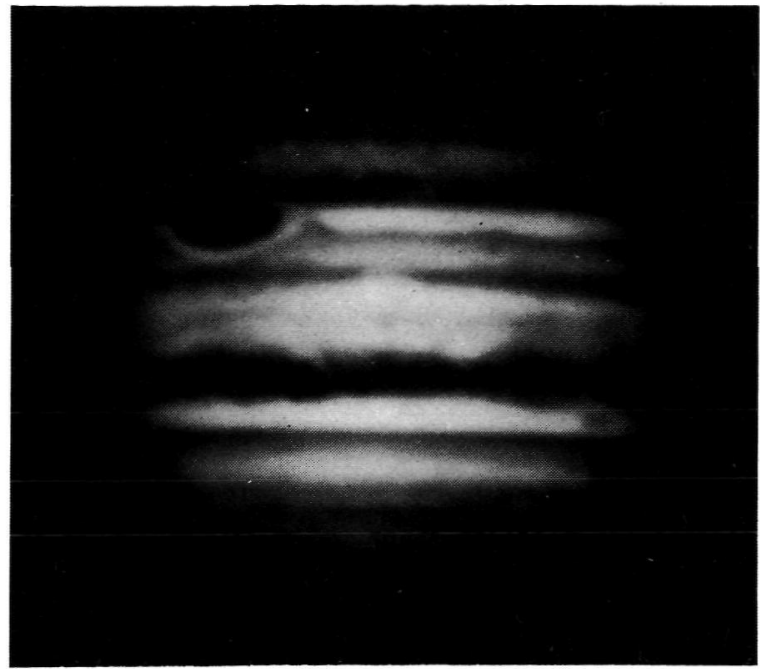

11 A photograph of the planet Jupiter, made with the 200-inch Palomar telescope. Taken in blue light, this photograph shows the banded structure of the cloud layer and the Great Red Spot. (Courtesy The Hale Observatories)

Moon, every one of them unknown territory. Three hours before transit, Discovery passed only twenty thousand miles from Europa, and all instruments were aimed at the approaching world, as it grew steadily in size, changed from globe to crescent, and swept swiftly sunward.

"Here were fourteen million square miles of land which, until this moment, had never been more than a pinhead in the mightiest telescope. They would race past it in minutes, and must make the most of the encounter, recording all the information they could. There would be months in which they could play it back at leisure."

From the novel, 200l: A Space Odyssey, by Arthur C. Clarke

Jupiter (Fig. 11) is the largest planet in the solar system. It is 11 times the diameter of the Earth and has more than 300 times the Earth's mass. It orbits the Sun at an average distance 5.2 times the Earth's. It is accompanied by a retinue of 12 satellites, four of which are comparable to or larger than our Moon.

The atmosphere of Jupiter is quite different from those of the inner planets-it is mostly the light gases hydrogen and helium, with small amounts of methane and ammonia. In fact, the composition of Jupiter's atmosphere (and probably of the entire planet) closely resembles that of the Sun. This is reflected in the fact that the average density of the planet is only 1.31 times that of water, compared to a value of 5.52 for the Earth and 1.25 for the Sun.

The visible surface of Jupiter is a cloud layer, believed to consist of frozen ammonia crystals. The 
visible regions of Ju piter's atmosphere are very cold $\left(135 \mathrm{~K}\right.$, or $\left.-220^{\circ} \mathrm{F}\right)$ due to the great distance of Jupiter from the Sun. However, the temperature is not quite as low as would be expected from the amount of sunlight received (about $105 \mathrm{~K}$, or $-270^{\circ} \mathrm{F}$ ), which is evidence that Jupiter is generating heat internally. This internal heat source is actually greater than the heat Jupiter receives from the Sun. It is believed due to very slow contraction of the planet (at a rate of only 1 millimeter, or 0.04 inches, per year), by which gravitational potential energy is converted to thermal kinetic energy. The interior of Jupiter is undoubtedly subject to great pressures and temperatures, reaching perhaps 30 million times Earth atmospheric pressure and temperatures of $10,000 \mathrm{~K}$ $\left(18,000^{\circ} \mathrm{F}\right)$ at the center. While small amounts of silicates and metals may exist, most of the interior is probably hydrogen and helium. At these temeratures and pressures, hydrogen could take on the form of a molten or solid metal. Further knowledge of the abundance of elements in Jupiter's interior and atmosphere, especially those of hydrogen and helium, is needed to decide between competing theories of the origin of the solar system and the universe.

Jupiter rotates very rapidly on its axis-once in just under 10 hours, faster than any other planet. The centrifugal force of this rapid rotation gives the planet a noticeably oblate shape-the equatorial diameter is some 7 percent larger than the polar diameter. The cloud patterns in the atmosphere form a series of bands parallel to the equator. These patterns show much fine structure, and a wide variety of color. One feature which appears to be more or less permanent is the "Great Red Spot," an oval feature larger in size than the Earth. Since the Great Red Spot (see Fig. 11) appears to move in longitude, and hence cannot be a solid surface feature, its nature is yet to be established.

Of the constituents of Jupiter's atmosphere, only ammonia and methane are readily detectable by ground-based techniques. Molecular hydrogen has only recently been observed, and is difficult to measure quantitatively. Helium is not detectable directly by any known ground-based method, nor are the other rare gases and nitrogen. Water vapor is not detected because of the extremely low temperature of the cloud layer, but is believed to be abundant in the warmer, unobservable levels below the clouds. Figure 12 shows a diagram of the cloud layer and temperature structure predicted for Jupiter's atmosphere.

Radio and Pioneer 10's observations of Jupiter have revealed the presence of a very intense zone of trapped high-energy electrons, analogous to the terrestrial Van Allen belts. The accompanying magnetic field is about 10,000 times stronger than Earth's. Direct measurements of the particle radiation and the magnetic field were made by the Pioneer 10 spacecraft. Figure 13 shows an early

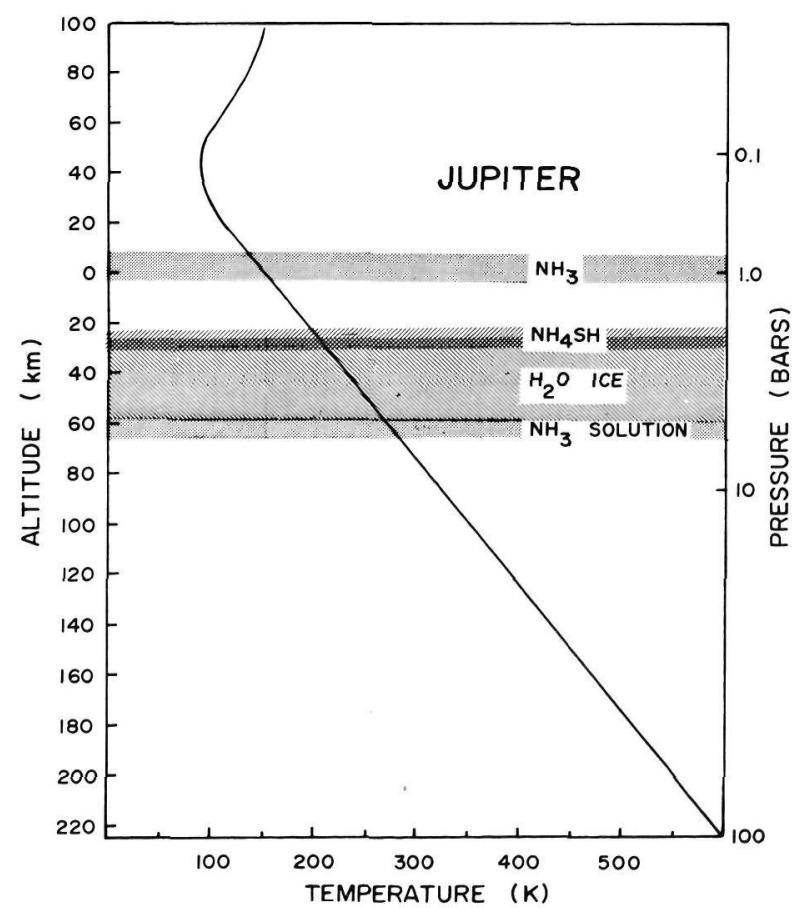

12 Diagram of the structure of Jupiter's atmosphere, showing the theoretically-expected variation of temperature and pressure with depth, and the altitudes and compositions conjectured for the cloud layers.

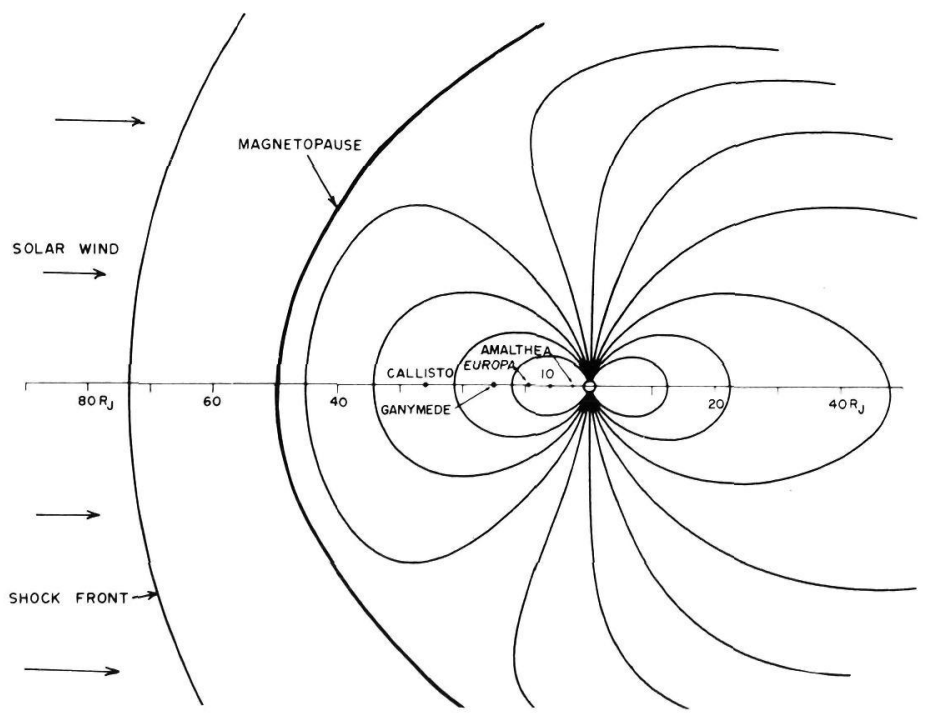

13 Hypothetical configuration of the magnetosphere of Jupiter prior to the analysis of Pioneer-10 data. Note that Jupiter's magnetosphere is much larger, even relative to the size of the planet, than that of the Earth, and that it includes the four major satellites. Pioneer 10 measurements showed that the magnetopause might be as much as 100 Jupiter radii $(\mathrm{Rj})$ ahead of the planet, twice as far as is shown in this figure, and that the magnetopause is much more complex than that shown here. 
idea for the probable configuration of Jupiter's magnetosphere based on the analogy with Earth's magnetosphere. The bow shock wave and magnetopause were both clearly measured by the plasma probe, magnetometer, and energetic particle detectors on Pioneer 10. The magnetopause was measured repeatedly, as if a Jovian magnetic storm were forcing the magnetosphere to move in and out several times.

The four major satellites discovered by Galileo in 1609, Io, Europa, Ganymede, and Callisto, are all comparable to our Moon in size (see Table 2). Ganymede is the largest satellite in the solar system, and is considerably larger than the planet Mercury (although not as massive). No atmospheres have yet been conclusively detected on any of the Galilean satellites. However, estimates of their densities and photometric ground-based measurements seem to indicate that they consist largely of ice. Ammonia may also be present as frozen water solution. Thus, these satellites are likely to be quite different in nature from our Moon. Recent ground-based observations of Ganymede, and Pioneer-10 measurements of Io, have indicated the possibility that very tenuous atmospheres appear to surround these satellites.

\section{SATURN}

"Discovery was now deep into the wide-ranging system of moons, and the great planet itself was less than a day ahead. The ship had long since passed the boundary set by outermost Phoebe, moving backward in a wildly eccentric orbit eight million miles from its primary. Ahead of it now lay Iapetus, Hyperion, Titan, Rhea, Dione, Tethys, Enceladus, Mimas, Janus-and the rings themselves. All the satellites showed a maze of surface detail in the telescope, and Bowman had relayed back to earth as many photographs as he could take. Titan alone-three thousand miles in diameter and as large as the planet Mercury-would occupy a survey team for months; he could give it, and all its cold companions, only the briefest of glances. There was no need for more; already he was quite certain that Iapetus was indeed his goal."

From the novel, 2001: A Space Odyssey, by Arthur C. Clarke

Saturn, the next planet out beyond Jupiter, is the second largest planet (Fig. 14). It is nearly 10 times the Earth's diameter and 95 times its mass. It rotates on its axis in just over 10 hours, slightly slower than Jupiter. However, its average density is less than that of water-the lowest density of any object in the solar sysfem. This is evidence that Saturn, like Jupiter, consists mostly of hydrogen

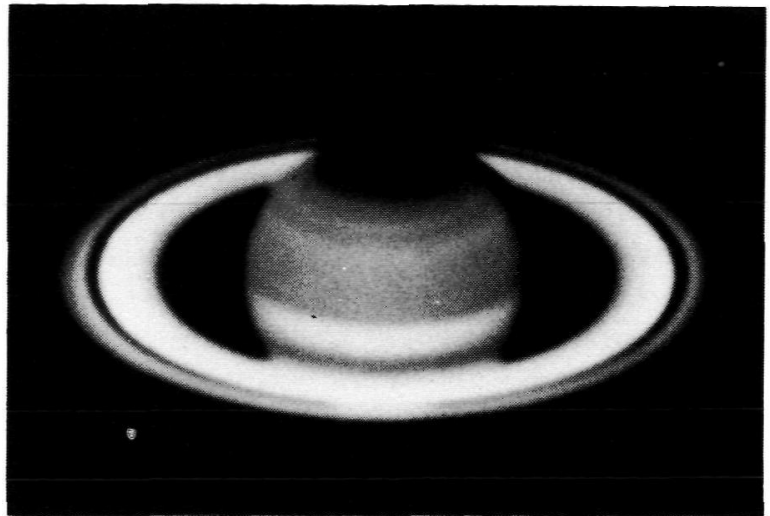

14 A photograph of the planet Saturn, obtained with the 200-inch Palomar telescope. (Courtesy The Hale Observatories)

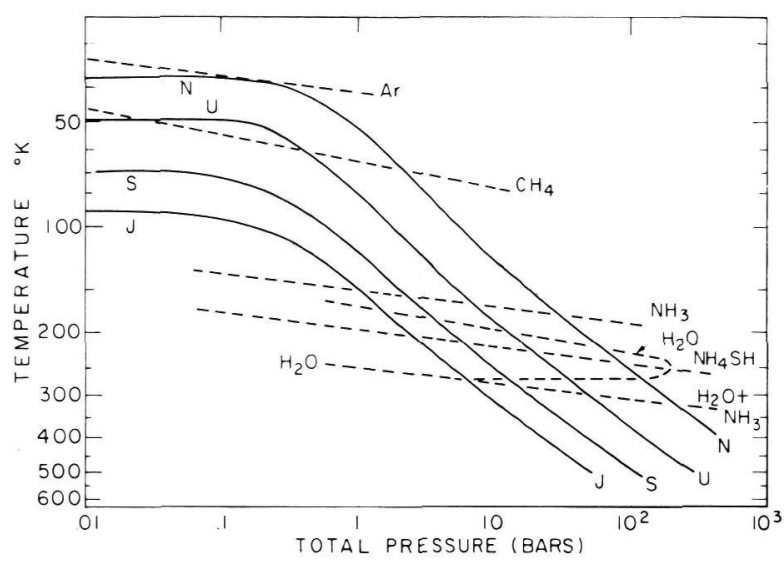

15 Atmospheric temperature profiles and cloud models of Jupiter, Saturn, Uranus, and Neptune, showing the condensation threshholds of methane $\left(\mathrm{CH}_{4}\right)$, ammonia $\left(\mathrm{NH}_{3}\right)$, ammonium hydrosulfide $\left(\mathrm{NH}_{4} \mathrm{SH}\right)$, water $\left(\mathrm{H}_{2} \mathrm{O}\right)$, and ammonia in aqueous solution $\left(\mathrm{H}_{2} \mathrm{O}+\mathrm{NH}_{3}\right)$.

and helium. Methane also is detectable in Saturn's atmosphere, but ammonia appears to be absent. This latter result is explained by Saturn's greater distance from the Sun (9.5 times that of the Earth), which causes the ammonia to be frozen out of the atmosphere, though the cloud layer probably consists of ammonia crystals as in the case of Jupiter. Saturn's cloud layer forms a banded pattern like that of Jupiter, but the contrasts and colors are much less marked.

Like Jupiter, Saturn appears to radiate more heat than it receives from the Sun, since its measured temperature of $97 \mathrm{~K}\left(-285^{\circ} \mathrm{F}\right)$ is considerably higher than the expected $70 \mathrm{~K}\left(-334^{\circ} \mathrm{F}\right)$. As in the case of Jupiter, this is evidence for an internal heat source.

Unlike Jupiter, however, Saturn emits no radio waves (normally generated from electrons trapped in a magnetosphere). Thus, Saturn lacks either a strong magnetic field or a source of electrons or, possibly, its rings (Fig. 14) may somehow prevent 
16 A photograph of Comet Tago-Sato-

Kosaka, which appeared in 1970. (Courtesy Cerro Tololo International Observatory)

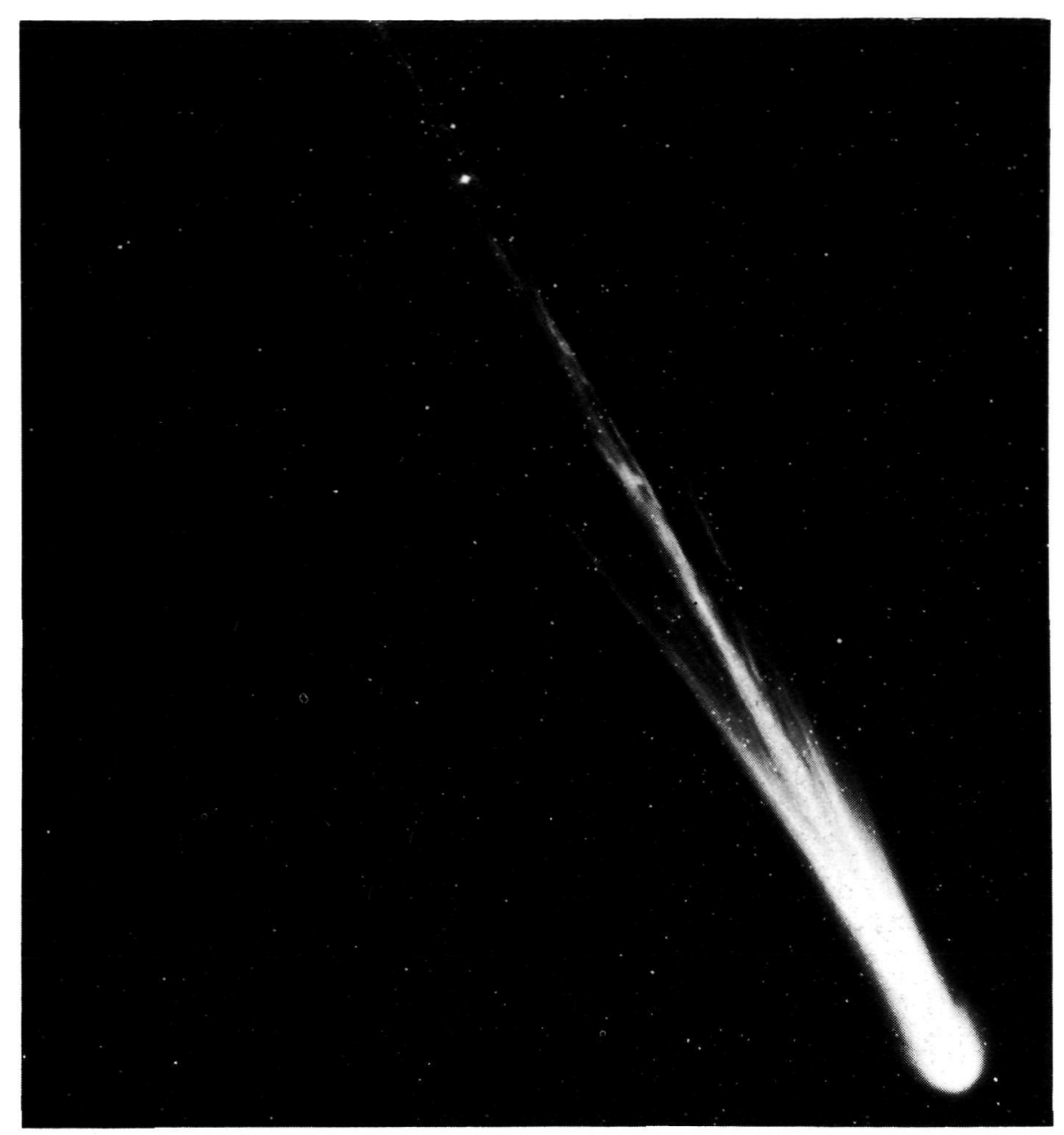

the formation of Van Allen belts. The rings are thin layers of particles orbiting in the equatorial plane of Saturn, extending out to about 2.3 times its radius. They exhibit zone structure, believed due to gravitational effects of Saturn's satellites. The maximum thickness of the ring system is believed to be less than 100 kilometers (60 miles), and its total mass is very small compared even to that of the satellites of Saturn.

The particles composing the rings consist at least in part of ice, based on ground-based infrared measurements. At the distance of Saturn from the Sun, ice does not evaporate appreciably, even over a time equal to the age of the solar system. The sizes of the particles are uncertain, but recent radar measurements have shown that at least some of the particles may be of the order of a meter in diameter or larger.

The satellites of Saturn are 10 in number, and the system is similar to that of Jupiter. Like the major satellites of Jupiter, many of Saturn's satellites seem to be composed largely of ice. The most interesting one, however, is Titan. This is the second largest satellite in the solar system, as large as the planet Mercury (but less massive), and is the only satellite known to have an appreciable atmosphere. The detectable atmosphere consists mainly of methane, although molecular hydrogen may also be present. There have been recent indications that clouds are present, and that the surface atmospheric pressure may be as high as that on Earth-and in any case, it is considerably denser than the atmosphere of Mars. The measured temperature of up to $160 \mathrm{~K}\left(-175^{\circ} \mathrm{F}\right)$ indicates a substantial "greenhouse" effect, as on Venus.

\section{URANUS AND NEPTUNE}

Each of these planets is about four times Earth's diameter and 15 to 17 times its mass. They are of intermediate density, consisting in part (but not mostly) of hydrogen and helium. Methane and molecular hydrogen are detected in their atmospheres, and the temperatures are extremely low due to their respective distance of 19 and 30 astronomical units (Earth-orbit radii) from the Sun.

Figure 15 shows the expected temperature profiles in the atmospheres of the four major outer planets. On Uranus and Neptune, condensed methane clouds can form (in addition to the ammonia 
clouds observed on Jupiter and Saturn). On Neptune, argon clouds may also be possible. Highresolution photographs of Uranus taken by the Stratoscope balloon-type telescope have shown that there are no detectable belt patterns parallel to the equator as on Jupiter and Saturn.

Uranus and Neptune are believed to consist of rocky cores of about one Earth mass, surrounded by several Earth masses of ices, and topped off by dense atmospheres of about solar composition. Neither is known to have a magnetic field or a trapped radiation belt. However, there is no theoretical reason to doubt the existence of magnetic fields, perhaps considerably stronger than Earth's, near these planets. Perhaps only direct measurements by space probes can settle this question.

Uranus has a regular system of five rather small satellites, while Neptune has one large satellite, Triton (larger than the Moon, but having no atmosphere which has been as yet detected) and one small satellite, Nereid. Triton is unusual in that it revolves around Neptune in the opposite direction from that of Neptune's rotation. Uranus and its satellite system are unusual, in that the plane of the satellites and of Uranus' equator are tilted more than 900 relative to the plane of Uranus' orbit around the Sun. Thus, at times, Uranus can present one of its poles almost directly toward the Sun.

\section{PLUTO}

This outermost planet of the solar system is unusual in a number of respects. It is small, perhaps not much larger than Mercury, but its mass is poorly known. No atmosphere has yet been detected. Pluto travels in a very eccentric orbit, so that at times it can be closer to the Sun than Neptune. An encounter between Pluto and Triton could have resulted in Pluto escaping from Neptune while simultaneously causing Triton to reverse its orbit direction. At present, Pluto can never approach Neptune closer than about 18 astronomical units.

\section{COMETS}

The cold, outer region of the solar sytem, beyond Pluto, is the realm of the comets. Occasionally, however, these spectacular celestial visitors (Fig. 16) sweep into the inner solar system-often approaching the Sun closer than the planet Mercury before rushing off again to the dark outer reaches, traveling on highly elongated, elliptical orbits. When observed in the inner solar system, a comet is seen to consist of three main parts. The main mass is in a nucleus of starlike appearance, ranging from 1 to 100 kilometers ( 0.6 to 60 miles) in diameter. Surrounding this is a nearly spherical cloud of diffuse luminous material, the coma, which extends out to several thousand kilometers from the nucleus. Finally, the tail is a luminous train which extends millions of kilometers from the comet head away from the Sun, gradually fading in brightness until it is no longer detectable. It is this tail which is responsible for the comet's name (from the Greek cometes, meaning longhaired).

As yet, the exact nature of comets is not entirely resolved. However, the most generally accepted model of the cometary nucleus is a conglomerate of ices and meteoric dust-literally, a "dirty snowball." The ices in this nucleus vaporize when the comet approaches the Sun, giving rise to the observed gases, and releasing the observed dust, which constitute the coma and tail of the comet. Some idea of the composition of the nucleus can be obtained by spectroscopic observations of light emitted by the coma and tail; various molecular fragments, or radicals $\left(\mathrm{OH}, \mathrm{CH}, \mathrm{CN}, \mathrm{NH}, \mathrm{C}_{2}\right.$, and others), which are not chemically stable under ordinary laboratory conditions, have been thus identified. If we assume that these radicals are produced by the breakup of "parent molecules" making up the cometary ices, it is deduced that these molecules consist largely of ordinary water ice $\left(\mathrm{H}_{2} \mathrm{O}\right)$, but in addition contain ammonia $\left(\mathrm{NH}_{3}\right)$, methane $\left(\mathrm{CH}_{4}\right)$ and more complex hydrocarbons, possibly also carbon dioxide $\left(\mathrm{CO}_{2}\right)$, nitrogen $\left(\mathrm{N}_{2}\right)$, cyanogen $\left(\mathrm{C}_{2} \mathrm{~N}_{2}\right)$, and other compounds of hydrogen, carbon, nitrogen, and oxygen. Some compounds are produced by cosmic-ray reactions with the major constituents at low temperature, and are unstable at Earth-like temperatures. Such potentially explosive materials may be responsible for cometary outbursts, or sudden enhancements of brightness.

From orbiting observatories, vast halos of atomic hydrogen have been observed surrounding the heads of some comets. Much larger than the visible coma (more than a million kilometers in diameter), the hydrogen halo is observed by its scattering of far-ultraviolet light from the Sun (of wavelength 121.6 nanometers $(\mathrm{nm})$, the so-called "Lyman-alpha" spectral line). This hydrogen halo, like the terrestrial hydrogen geocorona, is the result of the breakup by solar ultraviolet radiation (photodissociation) of water and other hydrogenous materials-but can be much larger, due to the greater vaporization rate and the weak gravity of the comet. Comet Bennett ejected hydrogen at a rate corresponding to the decomposition of 40 tons of water per second.

A comet gets brighter as it approaches the Sunnot only because of the greater intensity of solar 
radiation reflected, but also because of the greater rate at which gases and dust are ejected by the comet's nucleus. The solar wind (including its fluctuations and shock waves propagating in it) is another external factor which influences brightness. When a comet approaches very close to the Sun, one starts to see light emitted by vaporized metals near the head of the comet, as well as that of molecular radicals. These metals are presumably released by solar heating of dust particles, and include sodium and calcium. In the case of socalled "sungrazer" comets, which approach within a few solar radii of the Sun's surface, even vaporized iron, chromium, etc., are observed.

A non-gravitational force (evaluated through cometary trajectory studies) is believed to be the result of the jet reaction of gases boiled off the Sunfacing side of the nucleus. On occasion, this jet of gas has been observed to be at an angle with the Sun-comet line, evidencing rotation of the nucleus. Frequently, comets have been broken up by close passes of the Sun. The trajectories of comets are also affected by the gravitational attractions of the major planets (primarily Jupiter). For example, some "periodic" comets never go more than 4 or 5 astronomical units from the Sun-probably because their original, highly eccentric orbits were modified by close approaches to Jupiter.

The comet's tail is also of considerable physical significance. Actually, there are two different types of comet tails. The "dust tail" is composed of very fine solid particles, left behind from the "dirty snow" vaporized near the nucleus. This tail generally points directly away from the Sun, because the dust particles are driven radially outward by the pressure of sunlight. The "plasma tail" is composed of ionized gases, and is produced by the interaction of the solar wind with gases in the head of the comet. It was the fact that the plasma tail trails the head at an angle to the comet's trajectory different from that of the dust tail that led to the speculation (and later experimental verification) of the existence of the solar wind.

The composition of the comets is believed by some scientists to be representative of the original material from which the solar system was formed, exclusive of the most volatile gases such as hydrogen and helium, in which case comets are better preserved remnants of the original solar system, by virtue of the outer region "deep freeze," than are meteorites, asteroids, and lunar samples. More recently, however, other scientists have postulated that comets originate from interstellar material.

Since an average comet loses at least one percent of its mass at each close approach to the Sun, the fact that we still see them fairly frequently, 4.5 billion years after the creation of the solar system, is evidence that either there must be a vast number of comets in the outer solar system, or else that new comets are continuously being formed. Their number has been estimated at something of the order of 100 million, and their total mass to lie between $1 / 100$ and 1/10 the Earth's mass. The extent of the solar system's "cometary halo" may be an appreciable fraction of the distance to the nearest star.

\section{THE SUN}

The Sun is the central object of the solar system It has nearly 1000 times the mass of all the planets combined, and 300,000 times the mass of the Earth. Its diameter is about 100 times that of the Earth. However, the Sun is really only what astronomers would call an "average" star-there are stars hundreds of times the size of the Sun as well as others much smaller.

The Sun is composed of the same basic elements as is the Earth; however, the relative proportion of these are quite different. For example, about $80 \%$ of the mass of the Sun is hydrogen, the lightest element, and most of the remainder is helium, the second lightest. Heavier elements such as carbon, nitrogen, oxygen, silicon, etc., constitute only $1 \%$ of the Sun's mass. The composition of the Sun is similar to that of other stars, and of the interstellar gas. The giant planets, Jupiter and Saturn, are believed similar to the Sun in composition, and astronomers believe that the entire solar system was created about 4.5 billion years ago by gravitational collapse and condensation from a dense cloud of interstellar gas.

The Sun has a surface temperature of nearly $6000 \mathrm{~K}\left(11,000^{\circ} \mathrm{F}\right)$, with much higher temperatures below its surface (reaching more than 10,000$000 \mathrm{~K}$ at the center). The energy output of the Sun is equivalent to the complete conversion of nearly 5 million tons of matter into energy every second. For comparison, a 10-megaton hydrogen bomb explosion is equivalent to the conversion of slightly more than one pound of matter into energy. The process by which the Sun creates its energy, however, is very similar to that of the hydrogen bombit is a process of thermonuclear fusion, whose net result is the combination of four hydrogen nuclei to form a helium nucleus. The mass of hydrogen in the Sun is sufficient to supply its present output for a total time of about 10 billion years, so it has not yet lived half its total life.

The visible surface of the Sun, known as its photosphere, transmits most of the Sun's light and heat. The photosphere is not a true surface, but represents the lowest gas layer from which visible light can be radiated into space. There are other parts of the Sun which have less obvious but never- 
theless very important effects on the terrestrial environment. These include the lower atmosphere of the Sun, known as the chromosphere, extending a few thousand miles above its surface, and the outer atmosphere, or corona, extending several solar radii into space. These parts of the Sun are directly visible only during total eclipses or with special instrumentation, such as coronagraphs. However, they are the sources of most of the far ultraviolet and X-ray output of the Sun.

The corona is a very thin gas, but its temperature is extremely high-up to $2,000,000 \mathrm{~K}$. The reason it is so much hotter than the $6000 \mathrm{~K}$ photosphere may be that the heating is not provided by the photospheric radiation, but by shock waves propagating outward through the solar atmophere. These shock waves are produced by turbulent, convective motions in the lower atmosphere, evidenced in ground-based photographs by the granulated structure of the visible solar surface.

Figure 17 is a montage of a photograph of the solar corona, observed during a total eclipse of the Sun on March 7, 1970, and a photograph of the visible surface of the Sun (out of eclipse, near this time) in the light emitted by atomic hydrogen. Prominent features in the corona occur about active regions on the solar surface. The corona is also the source of the solar wind, which provides the interplanetary gas and magnetic field environment.

Solar flares, which are spectacular outbursts
17 A composite of a photograph of the solar corona, taken during a total eclipse, and of the disk of the Sun out of eclipse near that time. Note the correlation between coronal plumes and active areas on the solar surface. (Courtesy NOAA)

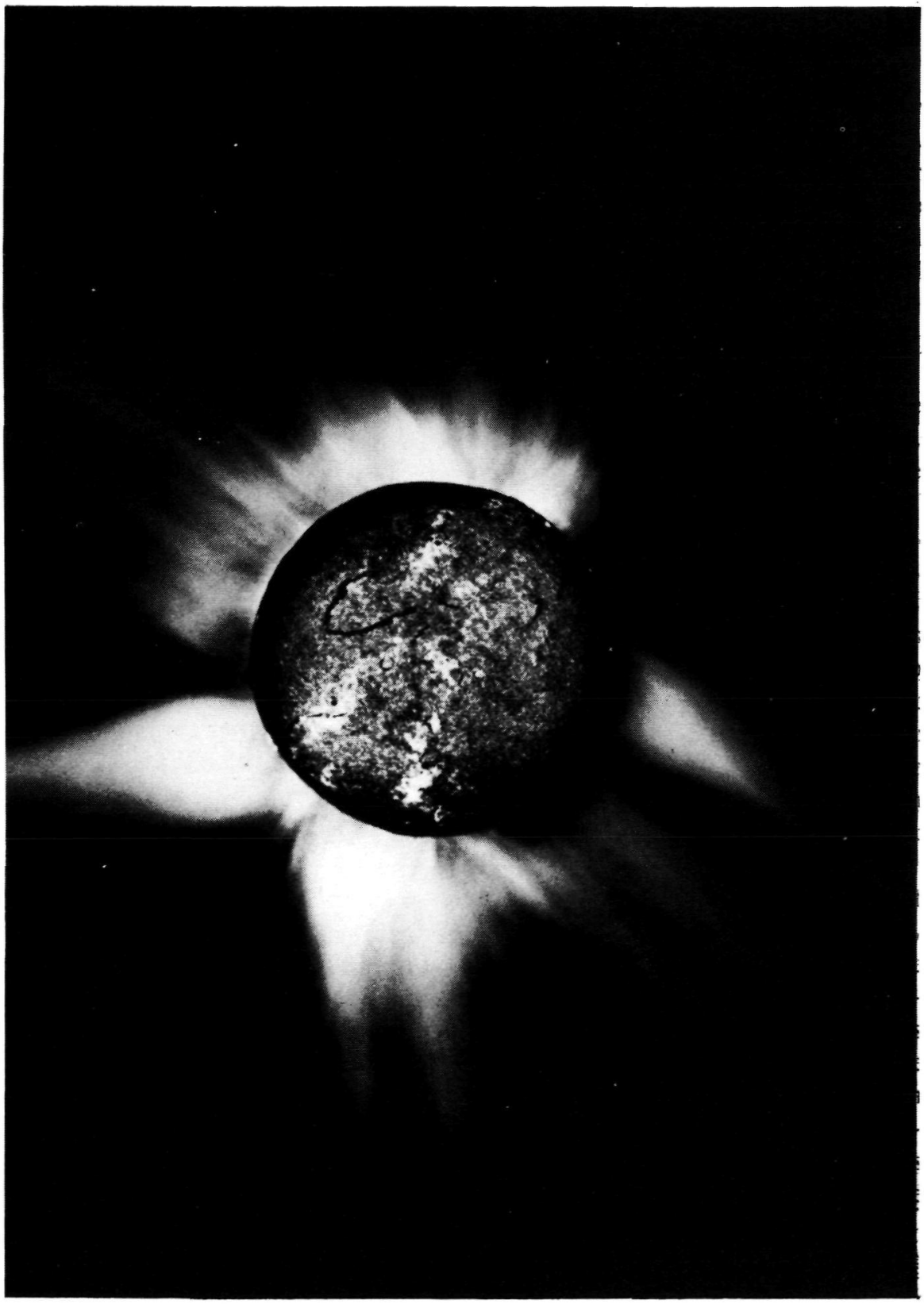


producing vast quantities of ultraviolet and X-rays as well as high-energy charged particles, occur in the upper chromosphere and lower corona. Flares generally occur near sunspots (regions of cooler gas in the solar photosphere). Such "active regions" are also the sources of prominences (jets of hot gas which sometimes shoot up high into the corona) and are characterized by locally intense magnetic fields.

The ultraviolet and X-rays emitted by the Sun are responsible for the terrestrial ionosphere, which is vital to long-distance radio communication. Another vital product of solar ultraviolet radiation is the terrestrial ozone layer. Ozone absorbs ultraviolet light and prevents it from reaching the surface of the Earth; without its protection, life as we know it would be difficult or impossible. Hence, variations of the solar ionizing radiation are of great practical importance.

Observations of these radiations require the use of rockets or space vehicles. The first such observations were made in 1946, using captured German V-2 rockets. These were followed, in the 1950's and continuing at present, with experiments carried on other sounding rockets, mostly smaller than the $\mathrm{V}-2$, although the experiments were continually improved in resolution and sensitivity. In the 1960's, the Orbiting Solar Observatory series of satellites was initiated, in which observations could be made over much longer periods of time than were possible with sounding rockets. Most recently, a highly advanced series of solar astronomy instruments was used in the Apollo Telescope Mount (ATM) package on board the Skylab space station. These studies complement groundbased visible light and radio observations. For example, they reveal processes in the high temperature regions of the atmosphere of the Sun and how they are related to events in the cooler regions closer to the solar surface.

Besides making it possible to observe the Sun in wavelengths inaccessible from the ground, space observations have increased our knowledge of the solar corona as observed in visible light. This results from the elimination of atmospheric scattering of light, which is responsible for the daytime blue sky. Thus, observing from above the atmosphere, we do not have to wait for an eclipse to observe the corona. More importantly, however, the space observations make possible much better observations than can be obtained even during an eclipse from the ground, as some scattered light is still present in the latter case. Rocket observations of the corona extend to much greater distances from the Sun than have been previously possible, and spacecraft observations have made possible studies of the time history of changes in the corona. These changes are directly related to changes in the solar wind and hence in the particle and magnetic field environment of the Earth.

Bursts of high-energy charged particles produced by solar flares and other solar disturbances interact with the Earth's magnetic field, producing disturbances to communications and electrical power transmissions. They are directly evidenced by the polar aurorae (northern lights), which are the result of the impact of high-energy electrically charged particles on the Earth's upper atmosphere. Such outbursts can also create a safety hazard to astronauts in space, and possibly even to passengers in high-flying aircraft. Therefore, it is of considerable practical interest to monitor the Sun for such disturbances, and to attempt to predict them.

\section{INTERPLANETARY GAS}

The gaseous material in the solar system can be regarded as an extension of the Sun's atmosphere. However, this gas is not at rest, but is continually streaming outward away from the Sunthe so-called "solar wind."

The Sun's outermost atmosphere or corona is very hot, having temperatures up to 2 million $\mathrm{K}$. Hence, even the immense gravity of the Sun is not able to permanently retain this extremely hot gas, which is an electrically neutral mixture of ionized atoms and free electrons called a plasma. This plasma is a very good electrical conductor, and hence has the property that the magnetic field lines of the Sun are constrained to move along with it. That is, the magnetic field is "frozen in" to the solar wind plasma.

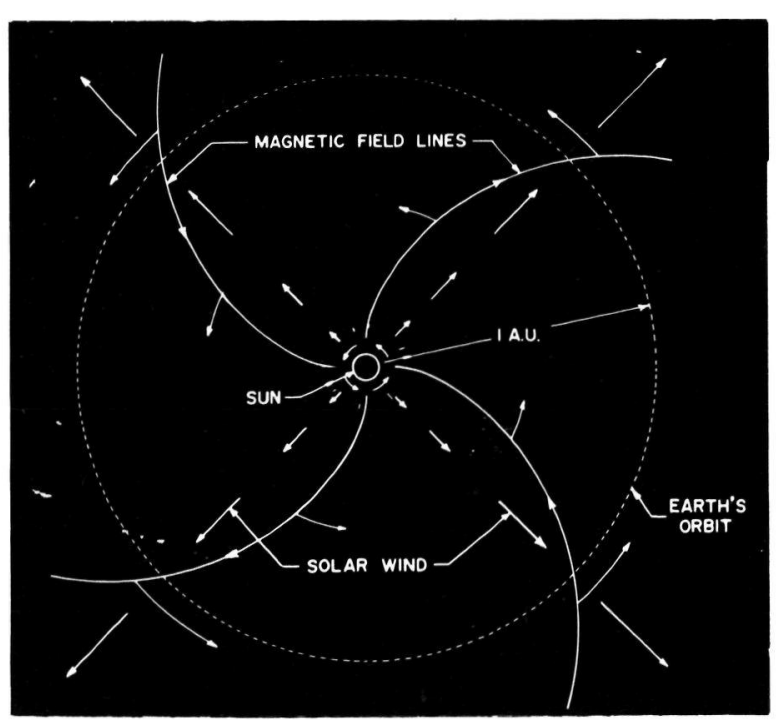

18 Schematic diagram of the Archimedean-spiral structure of the interplanetary magnetic field in the ecliptic plane. 
As the solar wind flows outward from the Sun, its velocity increases until a point is reached, about 5 or 6 solar radii out, where the velocity becomes equal to the speed of sound in the plasma. Then, the velocity becomes supersonic, and continues to increase-a three-dimensional analog of the process by which gas is accelerated to supersonic velocity in a rocket nozzle. Because of the solar rotation, which is a 27 -day period, the magnetic field lines have a transverse component, whereas the solar wind flow itself is essentially radial. Hence, the magnetic field lines take on the appearance of an Archimedean spiral (see Fig. 18). As one goes farther out in the solar system, the apparent direction of the magnetic field makes an increasingly larger angle to the line of sight to the Sun. At the Earth, this "garden hose" angle is about $45^{\circ}$. The velocity of the solar wind near the Earth is about $400 \mathrm{~km} / \mathrm{sec}(250 \mathrm{miles} / \mathrm{sec}$, or about 8 times the speed of sound in the gas), and its density is about 5 protons (and 5 electrons) per cubic centimeter ( 80 per cubic inch). For comparison, one cubic centimeter of sea-level air contains about $3 \times 10^{19}$ molecules. The composition of the gas is similar to that of the Sun, consisting of mostly protons (ionized hydrogen atoms), with lesser amounts of ionized helium, and very small amounts of heavier ions. These composition measurements have been made by foil collectors on several Apollo lunar surface missions and by instrumentation on various unmanned space probes.

Activity on or near the surface of the Sun can have marked effects on the solar wind. For example, solar flares release about half of their energy in the form of radiation and high-energy particles, and half as kinetic energy of the surrounding solar atmosphere in the form of a shock wave. This shock wave travels outward in the solar wind and can be very evident at the Earth, or even much beyond. Just as in the case of supersonic aircraftproduced shock waves in our atmosphere, this interplanetary shock wave is readily detected by sudden pressure, temperature, and velocity changes in the solar wind. Plasma probes, such as electrostatic analyzers and Faraday cups, and also magnetometers, have been used on interplanetary spacecraft to monitor these changes.

It is now a well-established fact that the Earth's magnetosphere deflects the solar wind, with the result that a standing shock wave is set up in front of the magnetosphere (as was shown in Fig. 2), directly analogous to the shock wave around a supersonic airplane. Pioneer 10 found a similar (but much larger) region around Jupiter, as shown in Fig. 13. Disturbances in the solar wind, as produced by flares, cause corresponding disturbances in the Earth's magnetosphere (magnetic storms). Such magnetic storms can cause communications disruptions and may even have appreciable effects on terrestrial weather. The interplanetary disturbances responsible for magnetic storms travel more slowly $(500$ to $1000 \mathrm{~km} / \mathrm{sec}$, or 300 to $600 \mathrm{miles} / \mathrm{sec}$ ) than the near-speed-oflight velocities of flare-ejected high-energy particles, but their consequences are equally important.

Interplanetary "weather" can be monitored by having spacecraft at several locations in the solar system, in the same way that terrestrial weather (or lunar seismic activity) is monitored by several widely spaced stations. Brightness fluctuations of comets appear to be related to solar wind disturbances, as may be some sporadic radio bursts from Jupiter.

As the solar wind flows outward in the solar system, its density must eventually decrease until it is equal to that of the surrounding interstellar gas, and/or its kinetic energy is less than that of the relative motion of the interstellar gas. The interstellar gas, like the Sun, is mostly hydrogen, plus about $10 \%$ helium (and less than 1\% heavier elements). Its density in the region of the galaxy near the Sun is about 0.1 atom $/ \mathrm{cm}^{3}(1.6$ atom/in 3$)$, and its velocity relative to the solar system is about 20 $\mathrm{km} / \mathrm{sec}$, or $12 \mathrm{miles} / \mathrm{sec}$ (based on recent observations from Orbiting Geophysical Observatory (OGO) and Orbiting Astronomical Observatory (OAO) satellites). Thus, it is expected that the solar wind eventually passes through a shock wave and is slowed to subsonic velocity.

The subsonic flow (shaded region in Fig. 19) is then believed to continue outward until it interacts with the interstellar gas in a region known as the "heliopause", in analogy to the "magnetopause", region between the Earth's magnetosphere and its

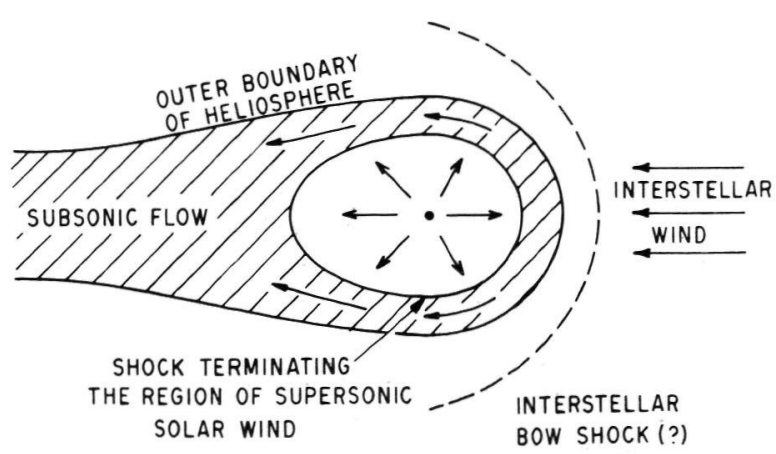

19 A diagram of the heliosphere. The region of supersonic solar wind is not symmetrical, being closer to the Sun in the direction from which the interstellar gas is approaching. The ionized component of the interstellar gas is excluded from the heliosphere, and if the relative velocity is supersonic, a "bow shock" may be formed as indicated by the dashed line. 
preceding shock front. Likewise, the region inside the heliopause is known as the "heliosphere."

The closest part of the heliopause is believed to be about 100 astronomical units (A.U.) from the Sun, well beyond the outermost planets. However, some interstellar gas can penetrate much further into the solar system. Neutral hydrogen can penetrate to about Jupiter's distance from the Sun before it is ionized by the solar wind and solar ultraviolet radiation; helium can penetrate to within less than the Earth's distance. Observations of ultraviolet light emitted by this gas, using sensors on OGO and Mariner spacecraft, permit determination of the direction and velocity of the interstellar gas motion relative to the solar system.

\section{THE ORIGIN OF THE SOLAR SYSTEM}

Our knowledge of, and conjectures on, the origin of the solar system are largely based on observations of solar system objects as they exist todaywhich differ (to a greater or lesser degree, depending on the object) from their state immediately following the formation of the solar system. Also, however, much information can be obtained from the study of other stars, and of clouds of dust and gas in interstellar space. Although we cannot study other stars and interstellar matter in as much detail as we can objects in our own solar system, we can observe, elsewhere in our Galaxy, objects in all stages of their life history-from prestellar contracting gas clouds to stars which have burned up their supplies of hydrogen and are in the final stages of their life cycles.

Such studies are of potential interest to mankind as a whole because they can provide a basis for estimating how many of the 100 billion stars in our Galaxy are accompanied by planetary systems. If even a very small fraction of such planets are habitable, there is a great potential for the development of intelligent civilizations elsewhere in our Galaxy.

At present, most astronomers appear to have accepted the theory that the Sun and planets were created about the same time, by the condensation of a cloud of interstellar gas and dust. Interstellar space is filled with dilute material having about the same overall composition as the Sun-mostly hydrogen and helium, with only about $1 \%$ by mass of heavier elements. The heavier elements are partially in the form of dust grains, but also are present in the gaseous phase and in the form of molecules. In fact, the interstellar dust bears a striking resemblance, in its properties and composition, to the material which makes up comets.

In regions of interstellar space where the gas density is high, the density of dust is believed to be higher in direct proportion. Thus, such regions are conspicuous in astronomical photographs because they block the light of distant stars behind them. Also, the hydrogen gas (normally in the atomic form) reverts to the molecular form, $\mathrm{H}_{2}$. Other molecules are also relatively more abundant in these regions. Species observed include water, ammonia, formaldehyde, carbon monoxide, and many other molecules and radicals. Such regions are also the places where new stars are observed in the process of formation.

Within our solar system, as we have seen, there is a striking trend in the variation of the properties of the planets going outward from the Sun. The inner planets are highly deficient in the lighter elements, particularly hydrogen and helium. More surprising, however, they are deficient in the heavier noble gases (neon, argon, krypton, and xenon) relative to their abundances in the Sun. Since these gases could not have escaped the gravity of the inner planets, this is evidence that these planets formed without any atmospheres at all-and that their present atmospheres are the product of outgassing of their interiors.

The most likely picture of the formation of the inner planets is that they were built up by agglomeration of planetesimals - each too small to retain gases by gravitational attraction. These planetesimals were built up by preferential condensation of the least volatile compounds in the primordial gas cloud (silicates, metal oxides, etc.). Therefore, the only volatiles present in these planets were those which could form solid compounds (such as hydrates) and do not depend on gravitational attraction to adhere to a solid body. Although the finished planets might have gravitational fields strong enough to retain other gases, they were unable to draw in such gases from the surrounding interplanetary gas because this gas was probably too thin and at too high a temperature (due to the simultaneous formation of the Sun nearby).

In the outer solar system, the lower prevalent temperatures allowed the planetesimals to retain a wider variety and greater proportion of volatilesincluding, for example, ammonia and methane (largely in combination with water ice). Hence the difference between comets and asteroids. Also, however, the lower temperature (and possibly higher density) of the surrounding interplanetary gas allowed sufficiently massive new-born planets to draw in hydrogen and helium and thereby further increase their masses. In the case of Jupiter and Saturn, this latter process apparently is responsible for most of the mass of these planets, since they seem to have the same relative proportions of hydrogen and helium to heavier elements as do the Sun and the interstellar medium. In the case of Uranus and Neptune, apparently the initial 
protoplanets were not sufficiently massive to enrich themselves in the light gases to as great an extent. The satellites of the major planets, like the comets, were too small to hold massive atmospheres, although they appear to be made up of the same "dirty ice" that formed the basis of the giant planets.

Since much of this picture of the formation of our solar system is based far more on theoretical conjecture than on direct measurement and observation, results of on-site exploration of the other planets will greatly refine or revise it.

\section{EXOBIOLOGY}

Important goals of biology and of man's explorations beyond the Earth are to determine whether suitable conditions for life are present elsewhere in the universe, and if so, whether life has, in fact, developed. We shall here present the evidence available at this time.

Life as we know it is based on the elements hydrogen, oxygen, nitrogen, and carbon. The temperature range for most active life is between the freezing and boiling points of water (273-373 K or $32-212^{\circ} \mathrm{F}$ ). Liquid water is essential for most terrestrial life, but molecular oxygen is not essential for some forms. Therefore, the planets Mars and Venus, which have detectable traces of water vapor and which, at least at some places and some times, have temperatures in the acceptable range, cannot be excluded as sites for life. The clouds of Venus might be habitable, as might some regions of the Martian surface. One missing link is the fact that nitrogen has not yet been detected on either planet. However, the sensitivity of the measurement techniques has been sufficiently poor that an adequate amount of nitrogen for biological processes cannot be excluded.

Of the other planets, surprisingly enough, the outer gas giants (and Saturn's satellite, Titan) are the ones where biologists would feel that low forms of life would be most likely to originate. At first glance, one would tend to rule out life there because of the low temperatures observed, and because of the presence of ammonia (poisonous to humans) and absence of molecular oxygen. However, as we have mentioned, molecular oxygen is not essential (and is, in fact, poisonous) to some microbial forms of life, and ammonia, methane, and water vapor contain all the essential elements for formation of organic compounds. Below the visible cloud layers of these planets, the temperatures rise to habitable levels, and water vapor (frozen out in the upper levels) is expected to be abundant. Laboratory experiments have shown that when mixtures of these gases (plus hydrogen sulfide, also believed present in the warmer regions of these atmospheres) are subjected to ultraviolet light or electrical discharges, complex organic compounds (including amino acids, the fundamental building blocks of proteins) are formed. Other experiments show that some micro-organisms can tolerate substantial amounts of ammonia.

Radio astronomical observations of dense gas clouds in interstellar space, similar to the gas cloud from which the solar system is believed to have formed, have revealed the presence of complex organic molecules. Also, recent analyses of a form of meteorite known as carbonaceous chondrite have shown the presence of amino acids-direct evidence for the presence of possible biological precursors in extraterrestrial material. Carbonaceous chond rites, like comets and the giant planets, are believed to be representative of the original material from which the solar system was created.

It has been said that if life of any kind is discovered elsewhere in our solar system, we can assume that wherever conditions amenable to the origin of life exist, it will occur, and, hence, that there almost certainly exist intelligent civilizations elsewhere in our Galaxy. There obviously is much to be done in exobiological investigations of the solar system. 


\section{Technology}

\section{A. Tools for Exploration}

The oldest method for space exploration is the use of optical telescopes to examine light reaching Earth from other bodies in the solar system and from the galaxies beyond. This technique suffers primarily from the disturbing influence of Earth's atmosphere, as well as from the physical limitations of optical instruments. Although the great distances between the Earth and these bodies limit resolution and brightness somewhat, the main problems are caused by the Earth's atmosphere, which blocks much of the spectrum over which we would like to observe these bodies, and whose turbulence severely limits the effective resolution of our instruments. Nevertheless, the bulk of our knowledge of the outer planets and the universe has originated from this source. Radio telescopes are also used to study electromagnetic waves and thermal emissions reaching our planet. Radar astronomy gathers valuable data from nearby objects but is relatively limited in range. Greatly improved images of these objects can be obtained by the use of telescopes observing from above the atmosphere (i.e., from Earth orbit, although rather good observations can also be obtained at balloon altitudes). The effects of atmospheric absorption on "seeing" over different wavelengths of the electronic spectrum are shown in Fig. 20.

Atmospheric turbulence usually limits the resolution of even the largest (200-inch) groundbased telescopes to about 1 second of arc, which is not even as good as the diffraction limit (the limit of resolution fixed by the capability of a given lens system) of a 12-inch telescope, although resolutions of 1.5 second of arc are sometimes achieved.

NASA has initiated a detailed design study of a 120-inch diffraction limited telescope (the Large Space Telescope, or LST; see Fig. 21) which would be launched aboard the space shuttle in 1980 or 1981. The LST has a resolution of 0.04 arc second, at least 10 times better than the best (200-inch) ground-based telescope, for an area resolution at least 100 times better than that of ground-based observations. For comparison, the maximum apparent angular diameter of Jupiter as seen from
Earth is about 40 arc seconds; of Uranus, 4 arc seconds, of Pluto, about 0.5 arc second. The major satellites of the outer planets also have maximum apparent diameters of order 1 arc second; i.e., although they are resolvable as discs in groundbased telescopes, any apparent markings are very indistinct. The LST, however, makes possible great advances in the imagery of these and other objects, such as asteroids and comets, as well as hard-to-observe stellar objects outside our solar system such as distant galaxies and nebulae. The LST will be particularly important for the more distant objects, which will not be viewed "close up" by space probes for a long time if ever. Also, unlike fly-by spacecraft, the LST can monitor changes in these objects over long periods of time. Even for Mars, which has been mapped at high resolution by Mariner 9 , this capability for long-term observation could be very valuable.

Already some of the benefits which might be obtained by the LST have been demonstrated by the Stratoscope program of Princeton University. Here, a diffraction-limited 36-inch telescope has been flown on a balloon to altitudes in excess of 24,000 meters $(80,000$ feet) for astronomical observations. High-resolution photographs much superior to previously available ones have been obtained of Jupiter, its satellite Io, and Uranus. In the case of Uranus, the Stratoscope observations have shown that there are no belts of clouds on Uranus, similar to those on Jupiter, although such had been reported by past observers. The Stratoscope has also been used for high-resolution imagery of the solar surface, revealing fine details in the granulation (believed to be convective upwellings of hot gas) observed there.

The LST also will be able to observe in electromagnetic wavelengths (far ultraviolet and far infrared) which cannot be observed from the Earth's surface, due to absorption in the Earth's atmosphere. Such observations can provide data on the composition and structure of planetary atmospheres and comets which are not obtainable by observations in visible light. These potentialities have already been demonstrated by soundingrocket ultraviolet observations of planets and ultraviolet observations with the Orbiting Astronomical Observatories, as well as close-up measurements by ultraviolet and infrared instrumentation on the Mariner spacecraft sent to Mars and Venus and on the Pioneer 10 and 11 Jupiter space probes to be discussed later.

The program of solar observations from satellites, begun with the Orbiting Solar Observatory (OSO) series in the 1960's, will continue with advanced OSO's in the mid and late 1970's. These observations should lead to the use of large solar 
telescopes for visible light, ultraviolet, $\mathrm{X}$-rays, and gamma rays on shuttle sortie flights. To perform such observations continuously for more than 30 days, a Large Solar Observatory should be placed in orbit in the 1980's.

More detailed measurements of such properties as total atmospheric composition, magnetic fields, and cloud-veiled surface features can be performed only by instruments carried to the near vicinity or surface of the planetary body by spacecraft. Early spacecraft missions to the Moon and the planets were the so-called flybys, which do not have the propulsive braking systems necessary for orbit. Although obviously limited in observa- of the density, distribution, and composition of the atmosphere.

Atmospheric probes, landers, and rovers can provide even more detailed information on the nature and extent of a planet's atmosphere and surface. The lander, of course, can combine atmospheric exploration with a survivable soft landing. It can then conduct experiments on the nature and extent of the surface and search for exobiological traces in its vicinity. Landers can also transport roving vehicles to the surface, to perform a variety of experiments in otherwise inaccessible and hazardous regions remote from the landed capsule.

Manned missions would permit far more ex-

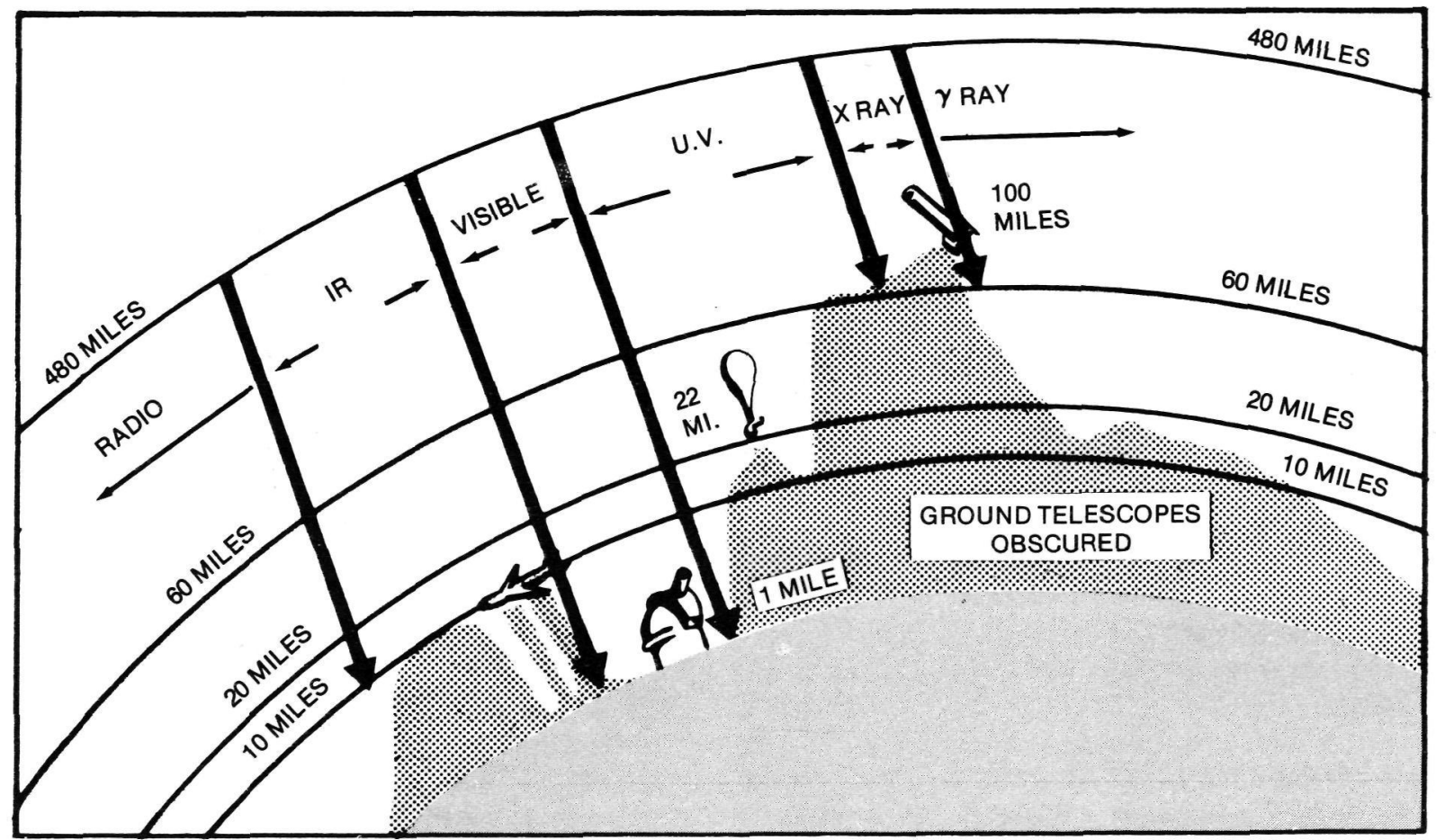

20 Blocking of radiation by the atmosphere, from radio to gamma-ray wavelengths.

tion time, these missions yielded important information about the nature of the atmosphere, particles and fields, and the magnetosphere (if any), and also provided visual images of the surface.

Orbiters are much more productive in collecting data, as seen in the Mariner 9 mission to Mars in 1971-72. Since planetary orbits can be designed to last for a number of years, it is possible to secure data on a continuing basis. Thus dynamic and seasonal changes can be surveyed by optical and onboard radar mapping as well as ultraviolet and infrared instrumentation, and long-term studies of the fields and particles of the planet can be made. Through occultation measurements, which determine the effect of an atmosphere on radio waves, orbiters (as well as flybys) also provide profiles tensive experiments to be made, but man's presence adds tremendously to the weight and complexity of the necessary operating and survival equipment.

\section{B. Spacecraft}

The two major types of spacecraft currently in NASA's stable for planetary exploration are the simple, spin-stabilized Pioneers and the three-axis stabilized Mariners. Together with selected propulsion systems they provide a set of proven, dependable and economical building blocks and have a promising growth potential for future solar system missions. Many cost-effective combinations are possible and can be tailored to specific mission requirements.

The present Pioneer role is that of a precursor, 


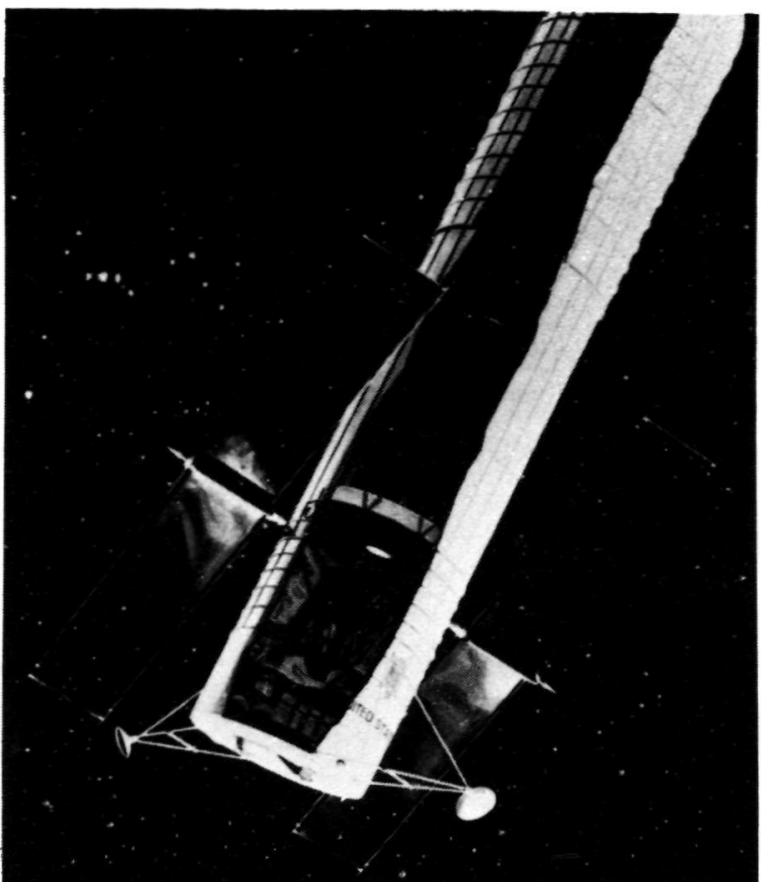

21 Large space telescope.

to chart the way for later more advanced spacecraft. Mariners provide a stable flyby and orbiter platform for photographic reconnaissance and spectral surveys. The Viking orbiter/lander spacecraft to be used for the exploration of Mars incorporates considerable Mariner technology; a fourth spacecraft type, Helios, is based substantially on the Pioneer series.

\section{Pioneer}

The characteristics of the basic spin stabilized Pioneer spacecraft are shown in Fig. 22, which also illustrates several aspects of their evolutionary growth. Although the basic configurations of all Pioneer-type spacecraft are similar, each mission

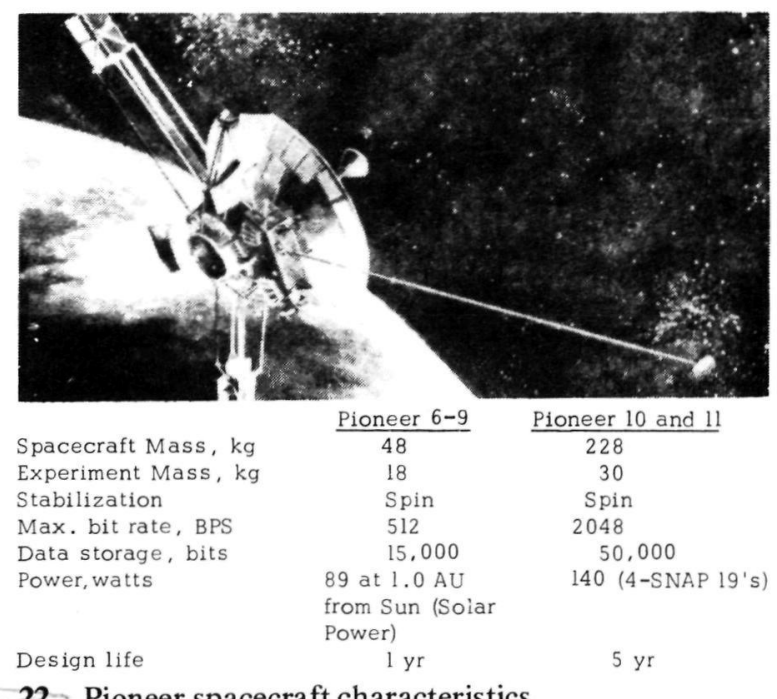

22. Pioneer spacecraft characteristics. can require different experiments and supportsystem characteristics.

The Pioneer 10 and 11 missions to Jupiter and beyond, for example, are precursors to exploration of the outer planets and a more complete understanding of the origin, history, and development of the solar system. These Pioneer spacecraft required considerable modification from the earlier Pioneers.

As an example, radioisotope thermoelectric generators (RTG's) using plutonium 238 have to be employed because solar cells are inefficient for missions so far from the Sun (see subsequent discussion in the section on "Launch Vehicles and Propulsion”).

The Pioneer 10 and 11 spacecraft (Fig. 23) each have a total power demand of 81 watts. They are equipped with four RTG's, which can produce about 140 watts.

Monopropellant hydrazine gas is used to spin the Pioneer spacecraft at five revolutions per minute when all appendages are fully deployed. Because these spacecraft are traveling to an unpre-

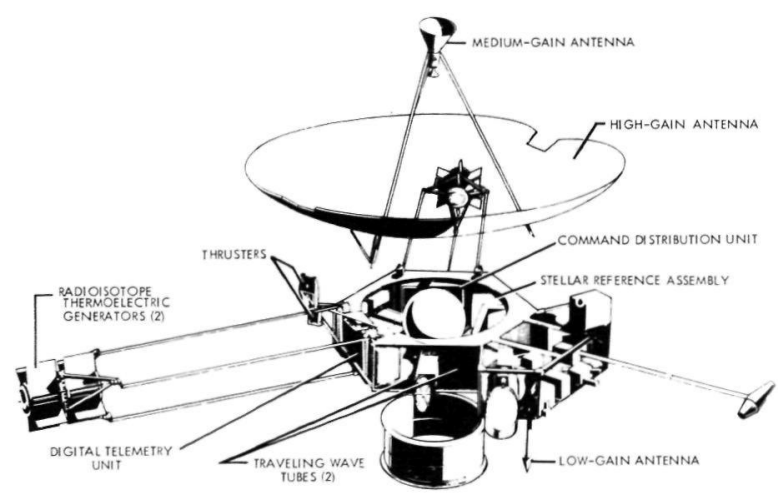

23 Pioneer 10 and 11 spacecraft configurations.

cedented 435-million-mile distance from Earth, a relatively large, 2.75-meter ( 9 foot)-diameter high-gain antenna is required to maintain contact with Earth and to transmit data at 1,024 bits per second (see glossary).

At such distances from the Sun, temperature control is an acute problem. A bi-metal louver system is used to conserve heat. Radioisotope heaters maintain the attitude-control gas bottles at a working temperature, and electric heating devices are installed on the gas lines.

Pioneer 10 was designed to perform 13 experiments, two of which required no special instrumentation (relying only on the radio transmitter): celestial mechanics measurements to provide data on the mass of Jupiter and a radio occultation experiment. Various shifts and delays in the signal 
provide information on the atmospheric heliumhydrogen ratio by indicating electron density, determine atmospheric temperature, and yield other data which pertain to the size of the planet.

Pioneer 10 also detected interplanetary shock waves generated by solar flares during the intense period of solar activity from August 2-11, 1972, when radio communication disruptions and other detectable events were recorded on Earth. Pioneer 10 , then at 2.2 astronomical units, was used together with Pioneer 9, Earth, and Comet Giacobini-Zinner to "track" these shock waves.

Pioneer 10 carries an imaging photopolarimeter with a spot field of view which sweeps with the spin of the spacecraft and builds a brightness profile that told experimenters much about cloud depth, striations, and other optical features of Jupiter's atmosphere. The device also looks at zodiacal light during cruise to provide information on particle distribution in interplanetary space. It formed pictures of Jupiter, with less detail than a TV picture (such as Mariner pictures of Mars), but nevertheless, at closest approach, much better than the best ground-based photographs.

Pioneer also had an infrared radiometer that yielded valuable data about the apparent thermal imbalance at Jupiter. An ultraviolet photometer determined the hydrogen-helium ratio which defines the major structure of Jupiter's atmosphere as compared with the Sun.

A group of eight instruments is used to investigate aspects of the particle and field environments in interplanetary space and near the big planets. One of these, a vector helium magnetometer mounted on a boom, also sampled the planetary magnetic field and provided indications of transition through Jupiter's shock wave as the solar wind moves into a Jovian magnetosheath. A plasma detector was used to measure ions near the planet and in the interstellar transfer zone.

A charged-particle detector records changes in plasma composition with increasing solar distance. Another detector measured the massive radiation belts at Jupiter while a Geiger telescope performed a charged-particle experiment near the planet. A cosmic ray telescope continues to observe changes in cosmic radiation as the spacecraft moves away from the Sun. Another experiment measures penetrations by micrometeoroids of various sizes. Finally, an optical detector records reflected light from passing particles, thus measuring their number, speed, and direction.

\section{Mariner}

The Mariner spacecraft's basic concept differs from that of the simpler spin-stabilized Pioneers in that it is stabilized on three axes. Its basic characteristics and evolutionary stages are shown in Fig. 24.

The experiments capable of being performed with Mariner spacecraft are typified by those carried on board the 1973 Venus-Mercury Mariner 10, shown in Fig. 25. These experiments are detailed in Fig. 26.

Visual imaging and an infrared radiometer are used to investigate the surface of Mercury and the cloud structure and atmospheric temperature of Venus. The ultraviolet spectrometer determines the presence and composition of an atmosphere. A charged particle telescope, plasma science experiment, and a magnetometer explore the interplanetary medium and the solar wind interaction with Mercury.

A detailed understanding of the interaction between the planet and the solar wind can yield important information about Mercury's characteristics. A radio science experiment searches for a Mercury ionosphere and determines pressure/tem-

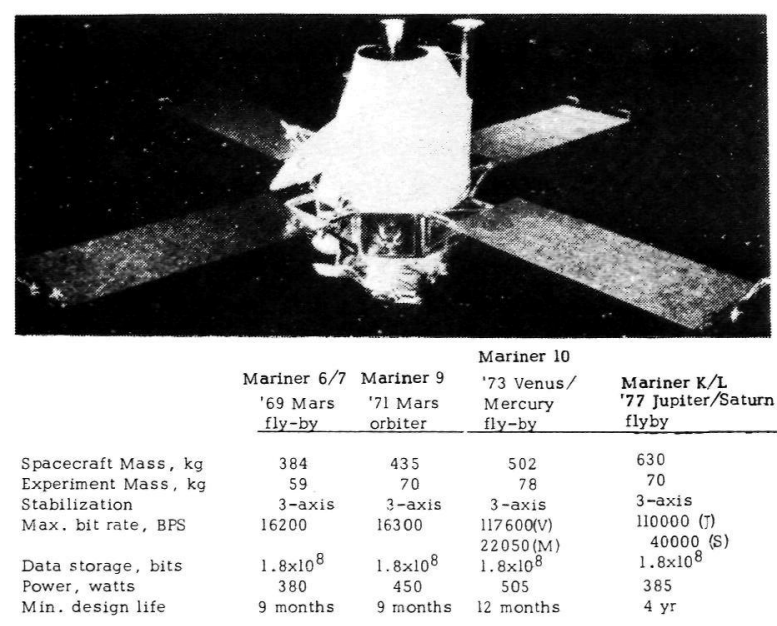

24 Mariner spacecraft characteristics.

perature profiles in a neutral atmosphere through a radio occultation experiment, in which radio signals traverse the planet's atmosphere as the spacecraft passes behind it and re-emerges on the other side.

The celestial mechanics experiment measures the planetary mass characteristics. It searches for clues in the gravitational field which might indicate the planet's departure from spherical symmetry or the presence of lunar-type mascons (mass concentrations below the surface, presumably resulting from meteor impacts). Extended tracking of the spacecraft during multiple re-encounters with Mercury could provide useful data on the solar gravity field.

During interplanetary cruise, the magnetome- 
ter, plasma science experiment, and charged particle telescope operate continuously, providing an excellent opportunity to gather data about the solar wind in the direction sunward of Earth, and to investigate its effects on magnetic fields between and around the planets. The ultraviolet spectrometer was used to measure some characteristics of Comet Kohoutek, as did Skylab instruments.

The Venus encounter in the mission was used primarily for the gravitational acceleration and trajectory modification needed to complete the flight to Mercury. This was, incidentally, the

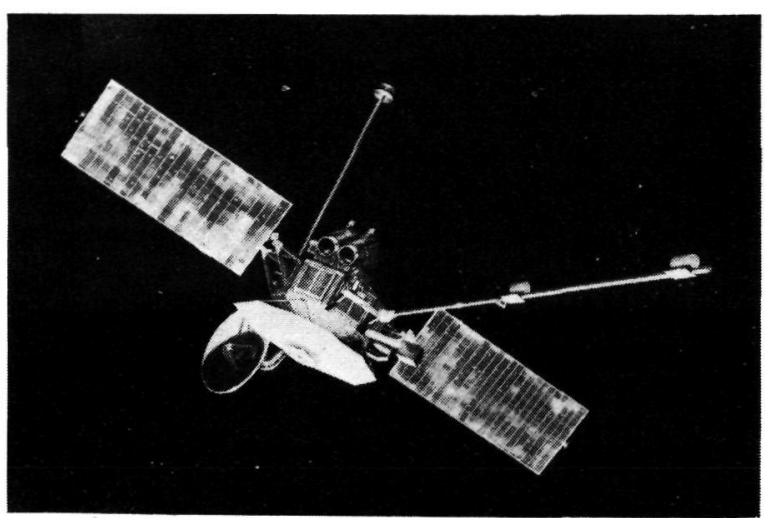

25 Mariner Venus-Mercury 1973 spacecraft.

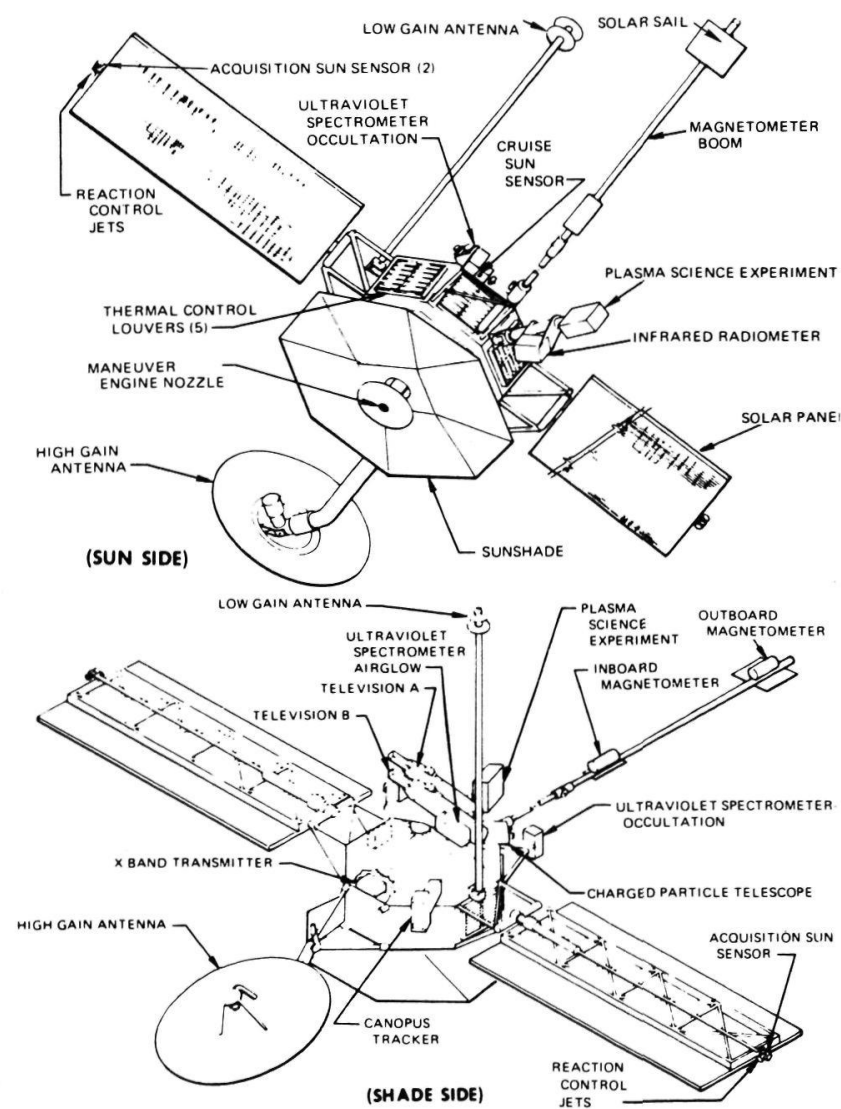

26 Venus-Mercury spacecraft experiments and subsystems. first mission to use the gravity of an intermediate planet to aiter the spacecraft's trajectory to the next planet. Science data taken at Venus include first-time photographs from a spacecraft to provide information on ultraviolet markings in the atmosphere and on the nature and spatial distribution of cloud stratification.

The clouds of Venus were photographed at the terminator (day-night shadow line) with wideangle cameras, while the spacecraft was at its closest approach. The infrared radiometer measured brightness temperatures at the tops of the thick cloud decks. The ultraviolet airglow spectrometer measured the abundances of hydrogen, helium, neon, argon, carbon, and oxygen. Using two frequencies, the radio science experiment probed the atmosphere down to about $35 \mathrm{~km}$ (22 miles) of the surface in order to refine the atmospheric model in temperature and pressure. Before encounter, the spacecraft flew on the opposite side of Venus from the Sun for several days, providing a unique opportunity to observe the solar wind in the wake of a planet.

The spacecraft was targeted for a dark-side closest approach to Mercury to provide an opportunity for the ultraviolet spectrometer to search for an atmosphere while the spacecraft passes into and emerges from the planet's shadow, and to allow optical measurements of solar wind interactions with the planet. The dark-side passage also provides measurements of the surface temperature variations as a function of distance from the terminator, using the infrared radiometer.

The Venus-Mercury spacecraft itself is based essentially on the Mariner 1969 and 1971 designs. The octagonal bus structure provides eight bays for electronic and other subsystems, with thermal control louvers on five of the bays. The locations of the propulsion subsystems allows control of the thrust direction without exposing the shaded side of the spacecraft. During the trajectory correction maneuvers, the spacecraft turns so that the louvers never look directly at the Sun. A large sunshade protects the electronic equipment, and solar panels tilt around the longitudinal axis as the spacecraft nears the Sun, in order to prevent overheating of the solar cells.

The solar panels provide a power output at Mercury of approximately 500 watts, about the amount required by the average home color television set. A high-gain, 54-inch-diameter antenna sends the data back to Earth. Television pictures containing more than five times the amount of detail found in home television pictures were transmitted back from Venus. Because of the longer distance, fewer pictures with this high resolution can be returned from Mercury. 


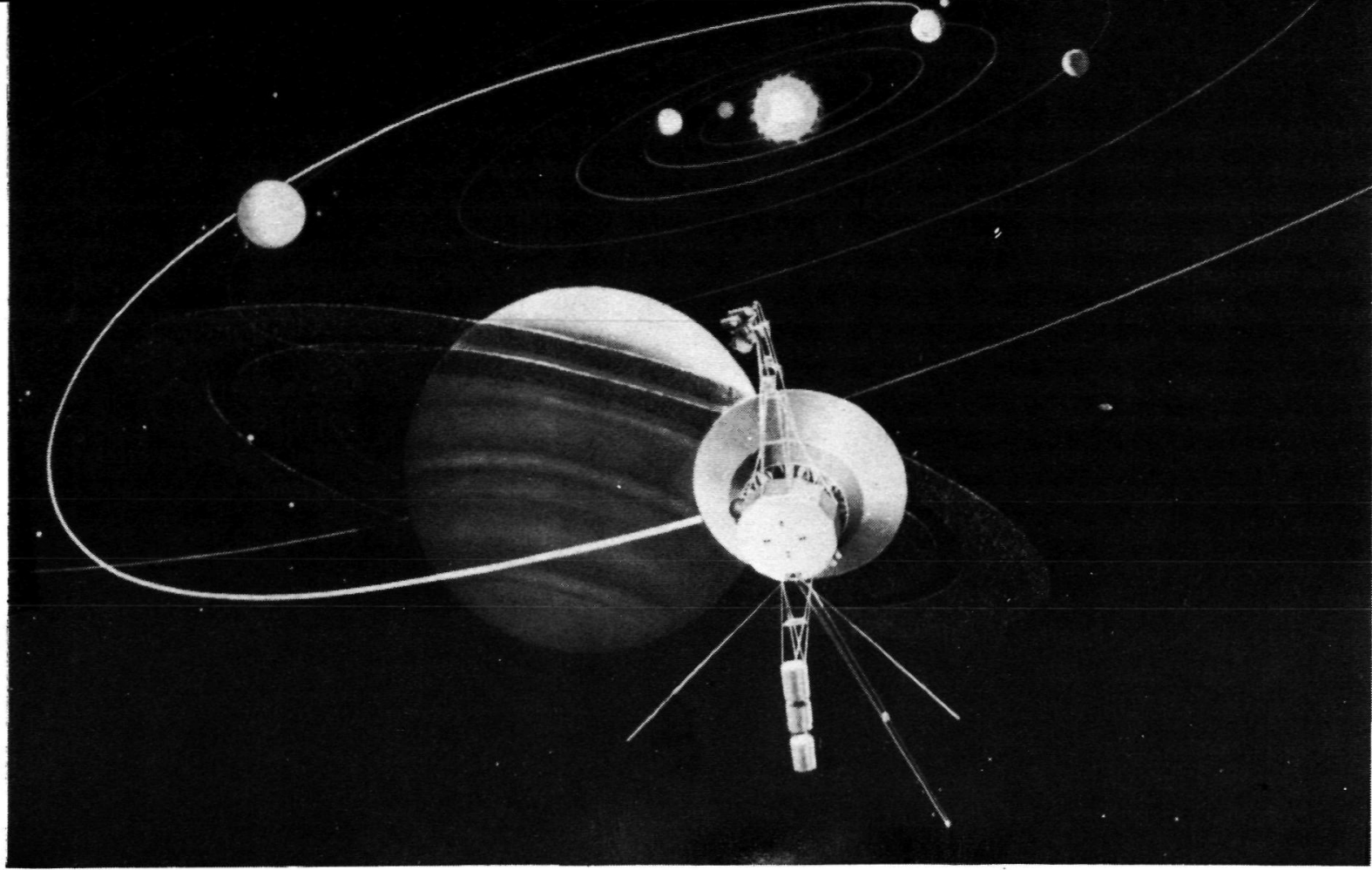

27 The Mariner Jupiter-Saturn 1977 spacecraft encounters Saturn.

The limited size of the propulsion subsystem and the large velocity relative to Mercury make it impracticable to slow the spacecraft sufficiently to put it into orbit about the planet. As with other Mariners, nitrogen gas provided by the attitude control subsystems stabilizes the vehicle along three axes. The Sun is used for the primary reference; Canopus, the brightest star near the south ecliptic pole, is the other reference.

The more advanced Mariner 1977 spacecraft destined for the exploration of Jupiter and Saturn (Fig. 27) is a direct inheritor of the technology used to explore the inner planets in earlier missions. The vehicle is stabilized on three axes with the Sun and the star Canopus still used as conven- ient positional references. The computer and data handling subsystems are very similar to earlier designs, although the performance of maneuvers and control of data rates are more critical: it takes 80 minutes to send a signal to the spacecraft at Saturn, another 80 minutes to verify reception and implementation.

The Jupiter-Saturn mission places extraordinary demands on spacecraft technology in several subsystems, primarily because of the greatly extended flight times, communications range, navigation precision, and high order of reliability required. Also, the time necessary for turnaround transmission to and from the Earth makes groundbased control impossible, necessitating the de-

TABLE 3

BIT RATE CAPABILITY

Spacecraft

Helios

Pioneer 10,11

Mariner 9

Mariner Venus-Mercury

Viking Orbiter

Viking Lander

Mariner Jupiter-Saturn

\section{Capability}

8 to 2,048 bps at 0.3 astronomical unit

\section{1,024 bps at Jupiter}

16,300 bps at Mars orbit

117,600 bps at Venus; 22,050 bps at Mercury

1,000 to 16,000 bps direct to Earth from Mars

4,000 to 16,000 bps relayed through Orbiter; 250 to 500 bps direct to Earth

110,000 bps at Jupiter $\approx 20,000$ bps at Saturn
Time to Send 1 TV Picture

$38 \mathrm{~min}$. *

1.3 hours *

$5 \mathrm{~min}$.

$40 \mathrm{sec}$.

$5 \mathrm{~min}$.

$5 \mathrm{~min}$.

$42 \mathrm{sec}$.

*No TV on spacecraft 


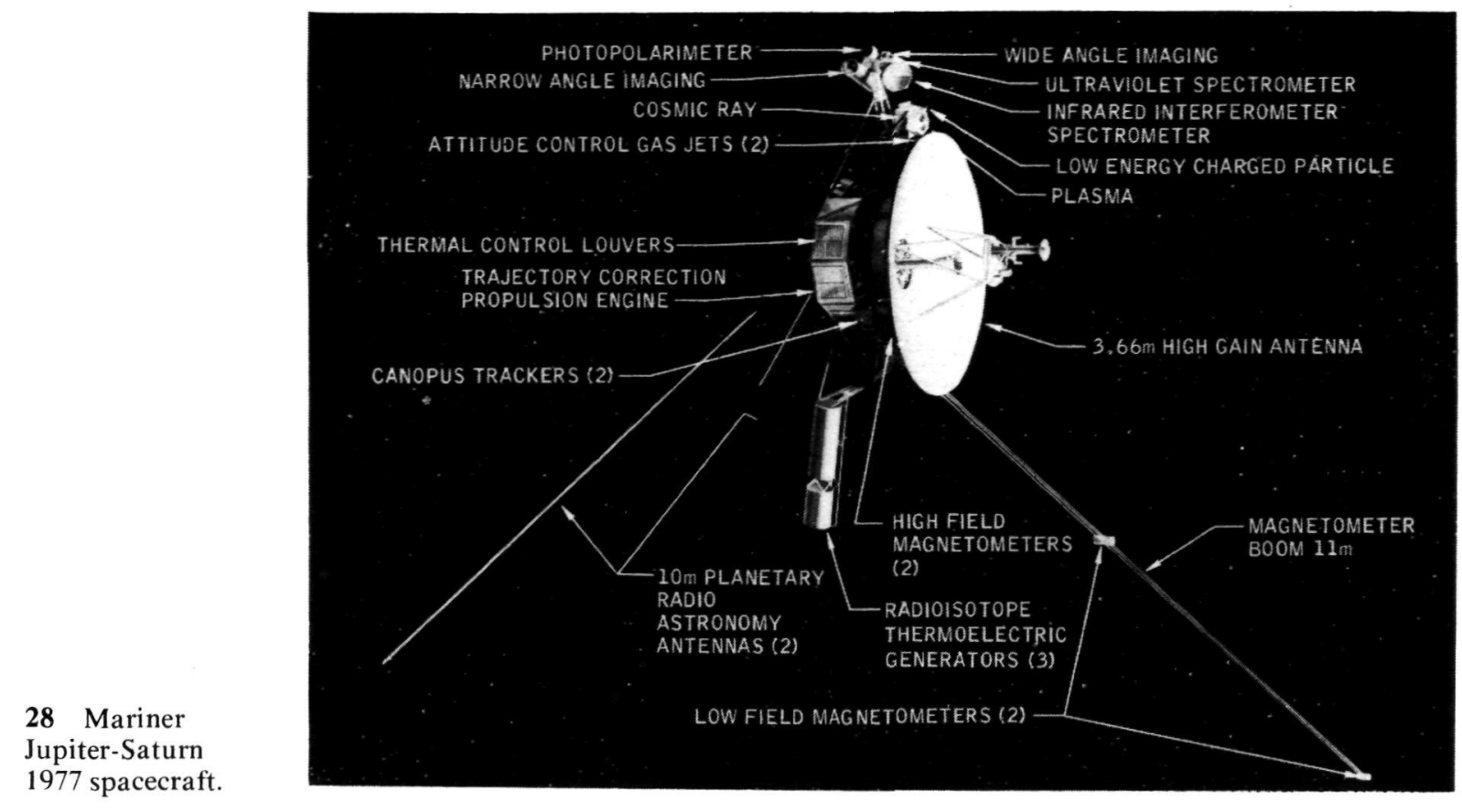

velopment of "artificial intelligence" for the spacecraft. The principal differences from earlier Mariner designs are in the power, propulsion, and communication subsystems (see Fig. 28).

As noted earlier in the discussion of Pioneers 10 and 11 , power systems based on direct solar energy conversion are impractical for outer planet missions, which are simply too far from the Sun. Thus, radioisotope generators will be used. The system being developed by the Atomic Energy Commission for the Mariner 1977 spacecraft is much larger than those on Pioneer 10 and 11, with a capability of about 130 watts per unit. The spacecraft will carry three of these 130 -watt generators.

The increased distance also demands much more efficient reception and transmission of radio signals to Earth-a three-fold increase in antenna size from earlier Mariner configurations. The system will use a higher (X-band) frequency, which permits the transmission of much more data at the same power level than the lower S-band frequency. With 0.2-degree pointing precision and a 24-watt power output, the system can transmit data at a rate of 110,000 bits per second from Jupiter and about 40,000 from Saturn (see Table 3).

The propulsion requirements on the Mariner 1977 spacecraft will be particularly critical. First, because of the gravity assist technique, the system must be capable of very precise velocity adjustments, both in magnitude and direction. Several maneuvers will be performed: to correct the launch errors, on approaching and leaving Jupiter, and others as the spacecraft nears Saturn. The Pioneer 10 data show that Mariner 1977 can pass safely through Jupiter's powerful radiation belts, but with little margin for error. Saturn's rings (see Chapter 3) pose a potential hazard for the spacecraft, since recent radar investigations indicate the presence of solid bodies a meter or more in size. The spacecraft trajectory will have to be adjusted to avoid this region.

\section{Viking}

The United States is planning a combined orbiter-lander mission to Mars in 1975, whose two identical unmanned Viking spacecraft (Fig. 29) will be launched during a 30-day period in August and September of that year. The 730 million- $\mathrm{km}$ (460 million-mile) flight will require nearly a year before reaching Mars on July 4, 1976, the bicentennial of the Declaration of Independence. The science objectives will be to increase our knowledge of Mars by direct measurements in the atmosphere and on the surface, both from orbit and from lander instruments. The emphasis will be on biological, chemical, and environmental factors pertaining to the past, present, or future potential for development of life forms.

The Viking spacecraft will be 4.9 meters (16 feet) high and 3.6 meters (12 feet) in diameter, excluding the 9.75 meter (32-foot) solar panels. The lander with its capsule has a mass of $1120 \mathrm{~kg}$ (2460 lbs), the orbiter $2340 \mathrm{~kg}$ (5140 lbs), including $1430 \mathrm{~kg}$ (3150 lbs) of propellant required to place the spacecraft in Mars orbit.

The orbiter will survey the pre-selected landing site and conduct scientific investigations of the atmosphere and surface of Mars before delivering 


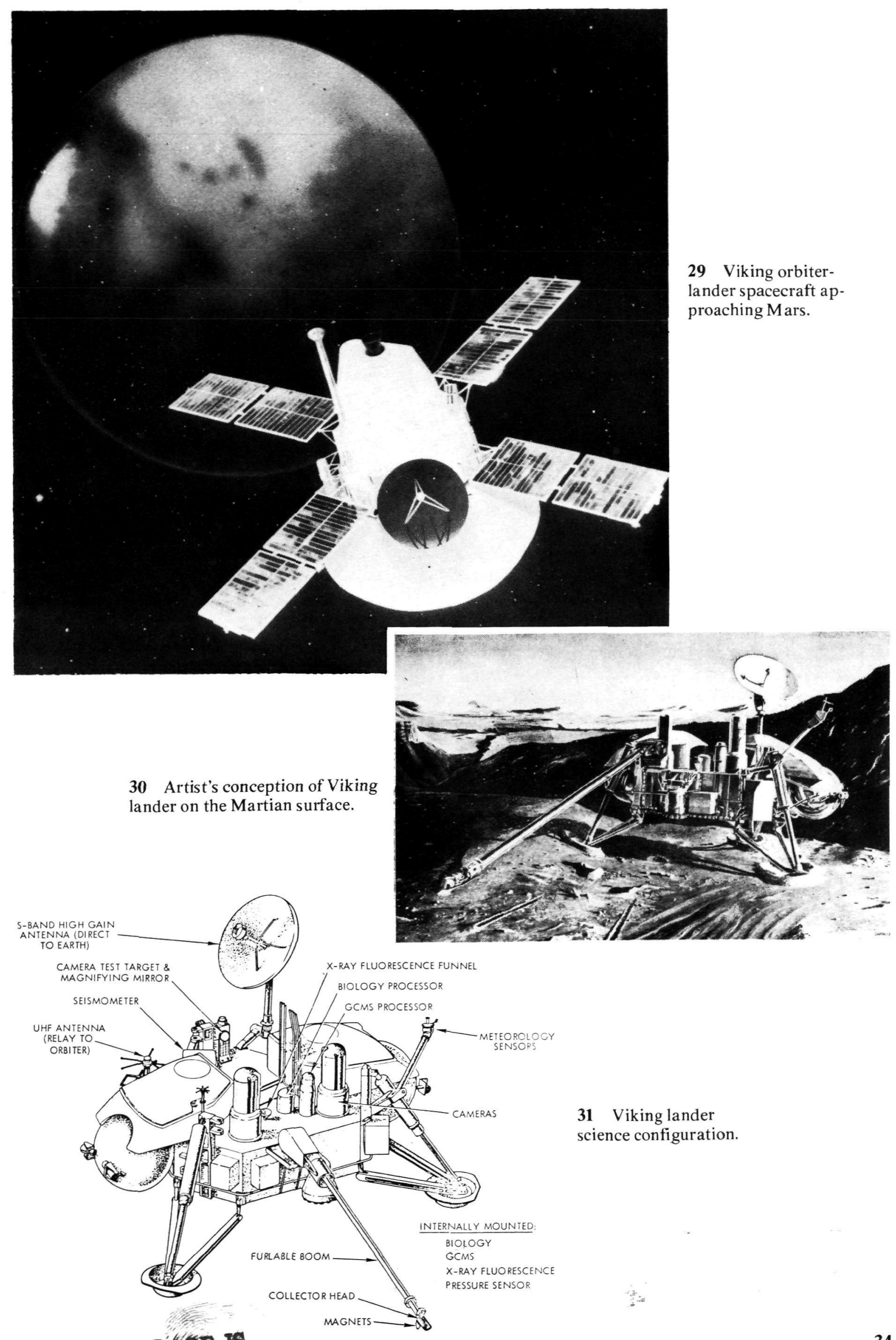




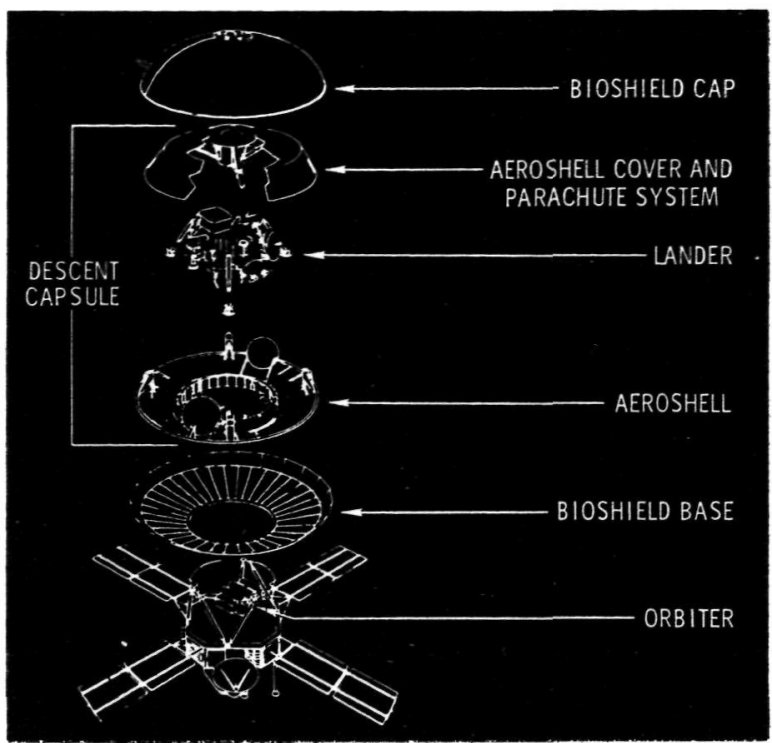

32 Exploded view of Viking spacecraft.

the lander (see Figs. 30 and 31) to the required separation point. Following the landing, the orbiter and lander will work together for a minimum of 90 days. During this period, the orbiter (Figs. 29 and 33) will relay to Earth the data originating from the landed experiments. The orbiter will also photograph the surface with its television cameras, with maximum capability during each revolution covering an area of about $80 \times 1050 \mathrm{~km}$ (50 x 650 miles) with 80 -meter (270-foot) resolution. It also will map the water vapor content of the atmosphere with an infrared spectrometer and con- duct thermal mapping with an infrared radiometer. The radio subsystem will be used to probe the atmosphere, ionosphere, and gravitational field.

At launch, the lander is enclosed in a double capsule. The outer cover is a bioshield consisting of a cap and base (Fig. 32) which seals off the sterile spacecraft to prevent contamination of the planet by micro-organisms from Earth. The entire configuration is heat-sterilized after sealing, ensuring a contamination probability of less than one chance in a million. The bioshield cap is jettisoned soon after the spacecraft is injected from Earth orbit into the transfer trajectory. The base is released in Mars orbit after the descent capsule separates from the orbiter. The lander is also protected by an aeroshell, consisting of a cover and a base, that acts as a heat shield to protect against high entry temperatures. It is jettisoned during the last phase of the landing maneuver.

The combined orbiter/lander vehicle (Figure 33) stays in orbit for at least 10 days in order to check out the performance of on-board subsystems and the condition of the landing site. Then the lander with its 94-kg (206-1b) instrument load separates and descends for a soft landing. During the descent to the Mars surface, the lander systems will make direct measurements of the physical and chemical properties of the atmosphere. The composition of the upper atmosphere will be investigated with a mass spectrometer; the structure of the atmosphere will be probed with pressure and temperature sensors, a radar altimeter, and ac-

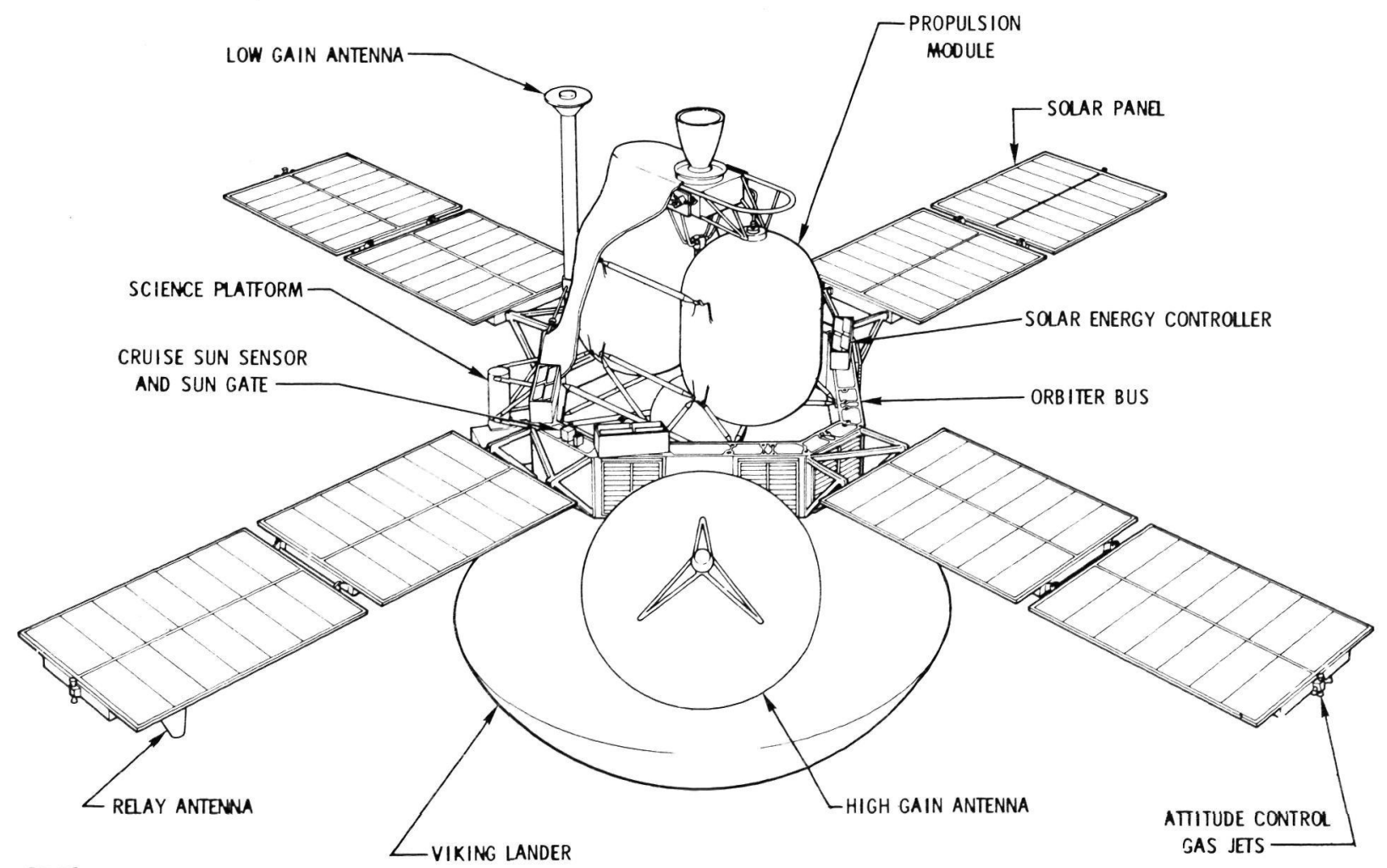

33. Viking spacecraft configuration, showing both orbiter and lander. 
netic properties of the surface by surveying soil samples for ferromagnetic particles.

The chemical properties of the soil and rocks in the vicinity of the lander will be analyzed by X-ray fluorescence, particularly for elements heavier than magnesium. The physical properties of the soil will be determined from data supplied by the imaging system, the surface sampler, and various engineering sensors mounted on the landed vehicle. The lander radar and telecommunication subsystems will be used in a study of the surface properties and to locate the lander on the Mars surface.

\section{Helios}

The Helios spacecraft are used to investigate phenomena very close to the Sun; e.g., at distances from 0.2 to 0.3 astronomical unit. The solar radiation intensity at 0.2 astronomical unit is 25 times its intensity at Earth, creating unprecedented engineering problems. The two spoolshaped, spin-stabilized Helios spacecraft, to be manufactured in Germany (Figs. 34 and 35), are solar powered and measure 2.75 meters ( 9 feet) in diameter by 4.2 meters ( 13.7 feet) high. They will spin at $60 \mathrm{rpm}$, with the spin axis perpendicular to the plane of the ecliptic.

C. Launch Vehicles and Propulsion

1. Launch Vehicles

Since the 1950's, scientific spacecraft have been

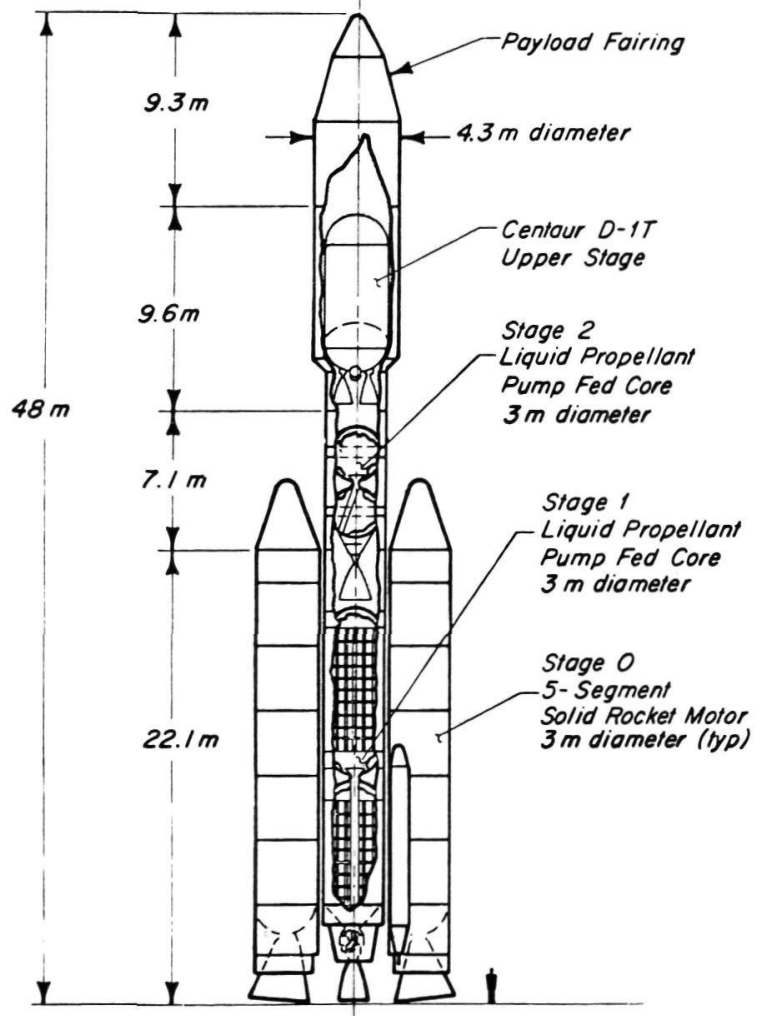

36 Titan III-E/Centaur expendable launch vehicle. carried aloft on their missions by liquid- and solid-propellant chemical rockets first derived, in most cases, from those used for military ballistic missile programs. For the next decade at least, these expendable launch vehicles (Table 4) will continue to provide for the mainstream of solar system exploration program. They can place between 200 and $15,000 \mathrm{~kg}$ (440 to $33,000 \mathrm{lbs}$ ) into low Earth orbits, and lesser payloads beyond. The Titan family represents recent technology and probably will be increasingly employed in the late 1970's. The Titan IIIE/Centaur (Fig. 36) will be used for the Viking 1975 Mars Orbiter/Lander and other major missions.

The space shuttle (Fig. 37), due to become operational in 1980, promises to provide a somewhat greater payload capability at lesser cost. In addition to possible launch economies, the shuttle brings two significant benefits to Earth-orbital science missions: (1) because of relaxed volume and mass constraints, experiments can be built more cheaply, and (2) scientists can accompany their experiments into space. In addition, such Earthorbital satellites as the advanced High Energy Astronomical Observatory (HEAO) and Large Space Telescope (LST) missions can benefit greatly from the multiple visit and "hands-on" approach opened up by shuttle availability. Shuttle benefits to interplanetary flight missions, however, are less clearly defined. A shuttle-borne interplanetary payload must still be carried from Earth orbit into its interplanetary trajectory by an additional rocket stage; however, the overall cost may still be less if a completely expendable launch vehicle were used. This additional rocket stage is usually expended, but use of electric propulsion during the interplanetary portion of the flight would in some cases allow a reusable tug to be the additional (recoverable) stage. Deep-space payloads must be reliable, rugged, and work unattended for years. Also, mass can be a strong constraint; only detailed cost/mass tradeoff studies can define the degree to which mass allowances can be relaxed for each specific set of mission requirements.

An advantage offered by the shuttle is that the payloads, spacecraft, and injection stages of interplanetary missions can be checked out to some degree in the space environment while still in Earth orbit, before starting the upper stage. If malfunctions are detected during checkout, it may be feasible to retrieve the payload or spacecraft back into the shuttle's cargo bay and either repair it in orbit or return it to ground facilities for more extensive repairs.

Another potential advantage, yet to be demonstrated, is that the large "boxcar" volume of the shuttle's cargo bay can accommodate larger pay- 
celerometers.

At approximately 6,000 meters $(20,000 \mathrm{ft})$, the lander's parachute is deployed and the aeroshell (heat shield) is jettisoned. At slightly more than a kilometer above the surface, its three retro-rockets will fire to slow the descent for a survivable soft landing. They shut off automatically at touchdown.

After touchdown the experiments on the lander in Fig. 31 will focus on the search for life forms, the nature of the environment, and the geological characteristics at the landing site. The slow-scan imaging subsystem will take pictures of the area in order to gather biological, geological, and meteorological data. Some of the pictures will be taken in color, others will be three-dimensional

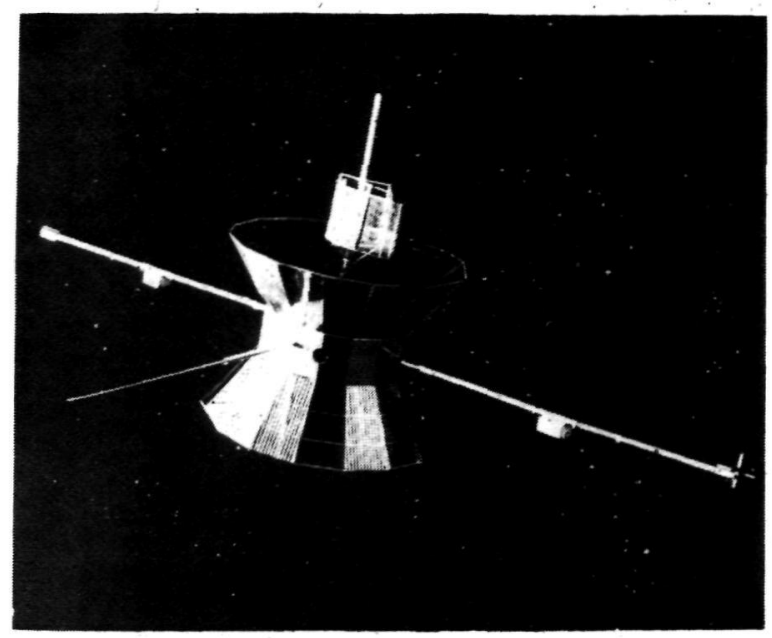

34 Model of Helios spacecraft.

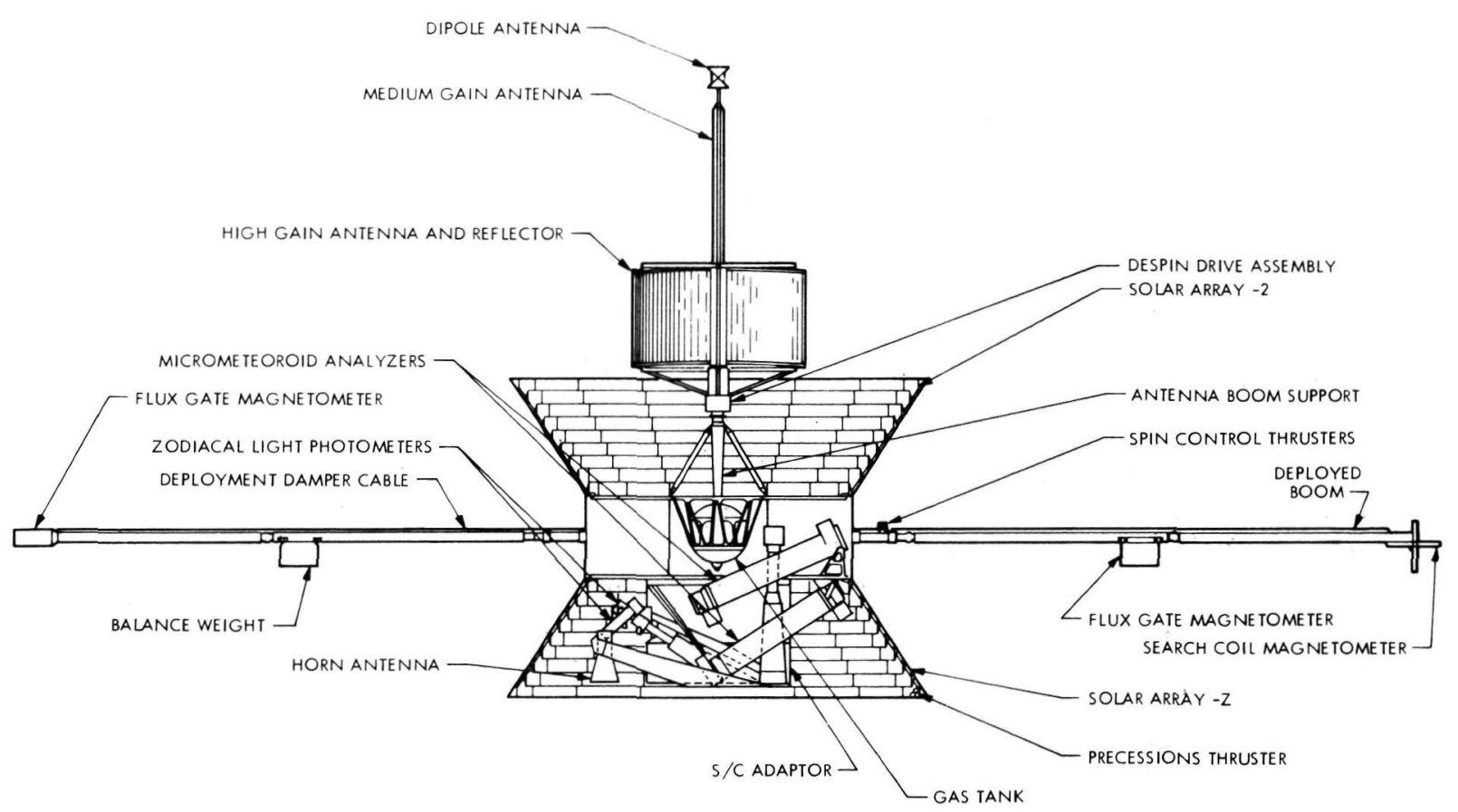

35 Helios spacecraft, showing principal elements.

stereo pairs produced by both facsimile cameras.

The biology tests will be based on three experiments, each searching for different physiological characteristics of life in the surface material in the immediate vicinity of the lander. A surface sampler will gather and deliver soil samples to the biological instruments of the lander for analysis. Tests for growth, metabolism, and photosynthesis will be conducted by an automated instrument package, using samples from the top $4 \mathrm{~cm}(11 / 2$ inches) of the surface material. A portion of the same soil samples will be used in the molecular analysis experiment to search for any organic molecules which are present in the soil. Samples of the soil will be heated in small ovens to liberate volatile gases which will then be separated by a gas chromatograph column and detected by a mass spectrometer. Water is one of the compounds which this instrument can detect. In another mode of operation, the mass spectrometer portion of the instrument will be used to measure the atmopheric constituents near the surface.

The lander also will be instrumented to measure the meteorological environment near the surface, including atmospheric pressure, temperature, and wind velocity and direction. A seismometer will seek to detect any measurable seismic events at the surface and to collect data from which the interior structure of the planet might be inferred. Another device will attempt to analyze the mag- 
SOLID ROCKET BOOSTER (SRB)

$3.6 \mathrm{M}$ (142.3 IN.) DIA

8.2 M (324 IN.) DIA

EXTERNAL TANK (ET)

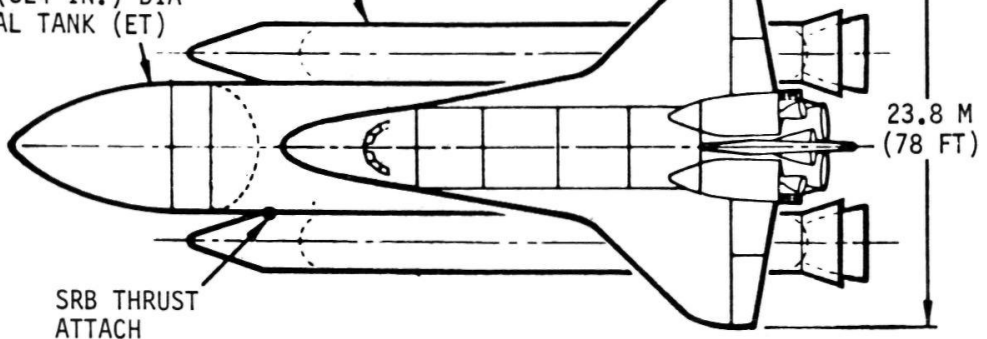

ATTACH

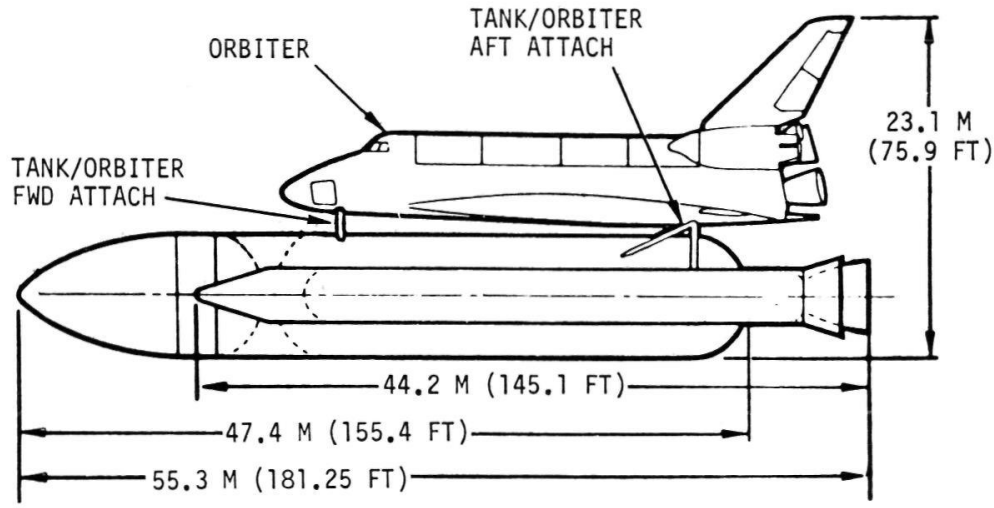

GROSS LIFT-OFF WEIGHT

$1889 \mathrm{~T}(4164 \mathrm{~K} \mathrm{LB})$

104 DEG INCLINATION

$93 \times 185 \mathrm{KM}(50 \times 100 \mathrm{~N} \mathrm{MI})$

ORBITER 68.T (150K LB) DRY

SRB $\quad 1056 \mathrm{~T}(2327 \mathrm{~K} \mathrm{LB})$

ET $\quad 740 \mathrm{~T}(1631 \mathrm{~K} \mathrm{LB})$

PAYLOAD $14.5 \mathrm{~T}(32 \mathrm{~K}$ LB)

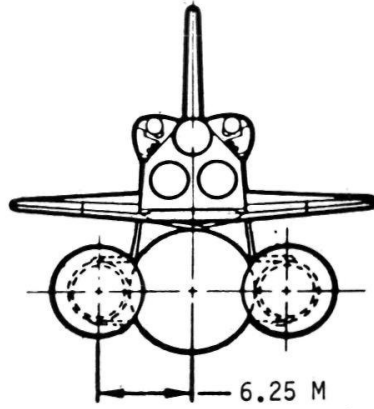

(20.25 FT)

37 Space shuttle configuration

Table 4 CURRENT AND NEAR-FUTURE LAUNCH VEHICLES USED FOR NASA AUTOMATED MISSIONS

\begin{tabular}{|c|c|c|c|c|c|c|}
\hline Launch Vehicle & Vehicle Status & $\begin{array}{l}\text { Total } \\
\text { Vehicle } \\
\text { Mass, } \\
\mathrm{kg}\end{array}$ & $\begin{array}{l}\text { Thrust Levels } \\
\text { At Zero } \\
\text { Altitude, } \\
\text { newtons }\end{array}$ & $\begin{array}{l}\text { Maximum } \\
\text { Diameter, } \\
\mathrm{m}\end{array}$ & Length, & Launch Site (s) \\
\hline $\begin{array}{l}\text { Scout* } \\
\text { Algol III } \\
\text { Castor II } \\
\text { Antares II } \\
\text { Altair III } \\
\text { Alcyone }\end{array}$ & Operational & 21,600 & 423,000 & 2.8 & 22.4 & $\begin{array}{l}\text { Wallops Island } \\
\text { Western Test Range (WTR) } \\
\text { San Marco }\end{array}$ \\
\hline $\begin{array}{l}\text { Delta }(2914) * * \\
9 \text { Castor Augmentation } \\
\text { Thor } \\
\text { Delta } \\
\text { TE364-4 }\end{array}$ & Operational & 132,000 & $2,100,000$ & 4.0 & 35.4 & $\begin{array}{l}\text { Eastern Test Range (ETR) } \\
\text { WTR }\end{array}$ \\
\hline $\begin{array}{l}\text { Atlas Centaur TE364-4*** } \\
\text { Atlas } \\
\text { Centaur } \\
\text { TE364-4 }\end{array}$ & Operational & 150,000 & $1,920,000$ & 4.8 & 39.8 & ETR \\
\hline $\begin{array}{l}\text { Titan IIIC } \\
\text { Zero Stage } \\
\text { Core I } \\
\text { Core II } \\
\text { Transtage }\end{array}$ & Operational & 632,000 & $9,880,000$ & 9.5 & 40.6 & ETR \\
\hline $\begin{array}{l}\text { Titan IIIE Centaur TE } 364-4 * * * \\
\text { Zero Stage } \\
\text { Core I } \\
\text { Core II } \\
\text { Centaur } \\
\text { TE364-4 }\end{array}$ & In Development & 640,000 & $9,880,000$ & 9.5 & 48.5 & ETR \\
\hline
\end{tabular}

*Usually flown as a 4-stage vehicle.

**Also flown with 3 or 6 Castor; when less performance is required.

**Also llown without TE364-4 upper stage

***Also flown without TE364-4 upper stage. 
loads than volume-limited aerodynamic fairings. Designers may use the larger volume to build in more redundancy to achieve longer lifetimes and reduce overall costs. Bigger and heavier spacecraft can be built more cheaply because there is less pressure for microminiaturization and close packing.

In the realm of more ambitious missions, the shuttle could be used to rendezvous with a returning sample-and-return vehicle. After a suitable period of quarantine the samples could be transferred to the shuttle and carried back to Earth, relieving the interplanetary vehicle of the complicated design required for Earth reentry.

\section{Spacecraft Propulsion}

Space propulsion engines are required for midcourse corrections and major trajectory changes such as planetary orbital injection. They can utilize either well-developed solid-propellant rocket engines, which are limited in that they can be used only once, or, more frequently, rocket engines using a liquid monopropellant (hydrazine) or storable liquid bipropellants (usually hydrazine and nitrogen tetroxide). For certain limitedenergy applications, simple gas jets utilizing prepressurized gases can sometimes be adequate. For the 1975 Viking lander a small throttleable liquidpropellant rocket engine will be used. It is quite similar to the larger one employed in the Apollo moon-landing vehicles, except that it must be automatically controlled.

The only spacecraft propulsion systems which have been used to date are based on storable chemical rocket principles. Electric propulsion is being considered for some future missions because of certain attractive features; it is particularly advantageous for the exploration of bodies with no appreciable gravity field, such as comets and asteroids.

\section{Advanced Technology Opportunities}

Research and development efforts expended through the space program have resulted in highly reliable and sophisticated propulsion systems and automated spacecraft. But while the present technology provides a basis for performing solar system exploration in the short term, it does not permit the type of balanced, advanced solar system exploration that might be hoped for in the 1980's and 1990's. For example, the present technology is strained to perform missions to Mercury, Jupiter, and Saturn, the many interesting comets and asteroids, and missions to regions well out of the ecliptic plane. Missions to the planets beyond Saturn, and to the major planetary satellites, as well as comet and asteroid rendezvous and planetary sample return missions, are either un- attractive due to cost and performance or impossible using present technology. If, indeed, solar system exploration is to proceed much beyond those goals which are already within our view, it will be necessary to provide commensurate programs in the further advancement of space systems technology.

The advancements necessary are in four areas: long-lifetime electronic equipment, autonomous “artificial intelligence," electric power systems, and propulsion systems. While it is clear that these technologies will have some applications in terrestrial programs, the time frame and peculiarities of solar system exploration will probably, and properly, provide the cutting edge of knowledge and development. Examples of particular technology development opportunities are discussed below.

\section{Component Reliability}

The primary requirement for some of the outer planet missions is long life for systems and instrumentation-on the order of 8-15 years. Current spacecraft such as Pioneers 6-9 and Vela have approached the lower limit of this span. These life expectancies can be achieved by the normal evolutionary improvement in electronic component design, in addition to appropriate thermal control of electronics compartments by such devices as heat pipes, long-life radioisotope heaters, selection of high-reliability parts, and redundancy design.

Communication system design for the long ranges of outer planet missions is essentially a compromise between transmitter power, system noise, temperature, and bandwidth, as well as antenna size and data rates. As the Thermoelectric Outer Planet Spacecraft (TOPS) study by the Jet Propulsion Laboratory has shown, by using 20-watt transmitting power and a 4.3-meter (14-foot) antenna, data rates of 131,000 bits per second are achievable at Jupiter (Mariner-JupiterSaturn is expected to produce $110,000 \mathrm{bps}$-see Table 3) and 2000 bits per second at Neptune.

The latter data rate assumes that data will be stored, and read out and transmitted to Earth periodically. Storage capacities of a billion bits are required, with ability to store and retrieve data át several bit rates in order to adjust to mission requirements. Tape recorders have been proposed as cost effective, but further development to overcome the wearout failure mechanisms inherent to mechanical devices is required to reach the 10-12 year life required. Solid state memories, in which one million bits are stored in a $2.5-\mathrm{cm}$ (1-inch) square, $.80 \mathrm{~mm}(1 / 32$ inch) thick chip, have been tested in the laboratory.

Perhaps the major development required in the 


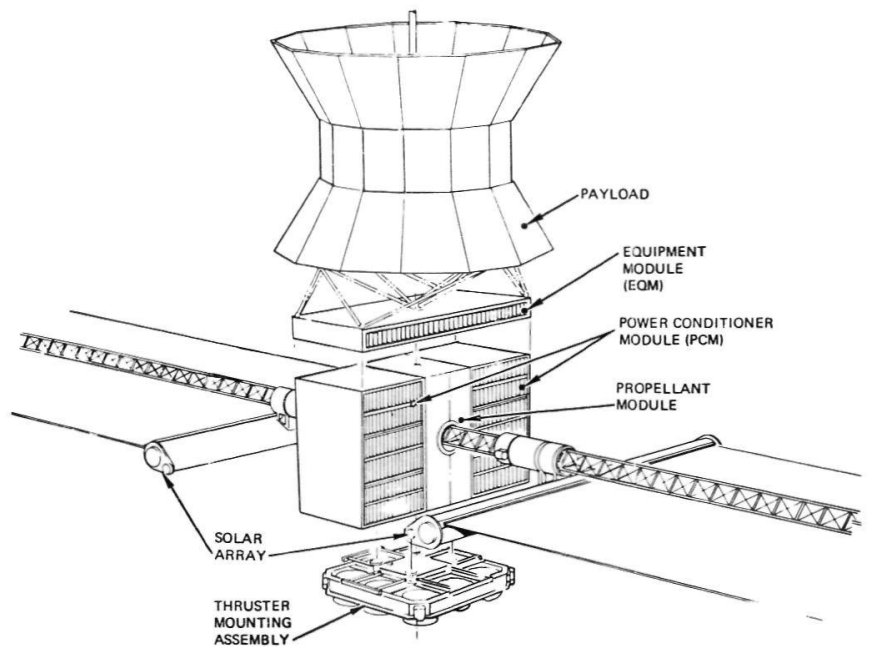

38 Solar electric propulsion stage (SEP) conceptual definition.

communication area is the continued development of the power amplifier. A 20-watt X-band traveling-wave tube is required, with high efficiency and the capability of operating unattended for 10 years. This life has been already demonstrated in lower level tubes (Pioneer 6, for example), and the confidence gained in this area from operational commercial communications satellites appears to warrant some optimism for successful future development.

Long transmission times constrain command and control. At Jupiter distances it would take up to 1-1/2 hours for news of a spacecraft malfunction to be sent to Earth and a corrective command to be sent and received by the spacecraft. Spacecraft should be designed to fail into a "safe" mode, allowing ground controllers ample time to diagnose the malfunction and take corrective action. Alternatively, a design similar to a Self-Test and Repair (STAR) computer studied by the TOPS design group might be utilized. Although there is much appeal to this approach, the major development program required is not being carried forward at this time.

\section{Space Electric Power}

Two sources of energy available in space, solar and nuclear, have received considerable attention for providing electric power to solar system exploration spacecraft. Photovoltaic cells, arranged in a device called a solar array, convert electromagnetic energy provided by the Sun into electricity; the use of reflectors to condense the solar energy for use in a thermodynamic cycle has been considered but never developed. However, considerable effort is going into the development of advanced solar arrays, especially in the reduction of solar array costs (presently about $\$ 300 /$ watt) and in reducing cell degradation, thereby increasing life- time and reducing solar array mass per watt output. One contending concept consists of a flexible, roll-up array. Its integration into an electrically propelled spacecraft is shown in the electric propulsion system illustrated in Fig. 38 .

Solar arrays derive their energy input from the Sun. At the Earth's orbit this energy is about 1.5 kilowatts per square meter; but it decreases as the square of the distance from the Sun, so that at Jupiter it has dropped by a factor of 30 and at Saturn by a factor of 100 . Thus, for exploration of the outer planets, it is necessary to rely on nuclear power sources.

Nuclear power sources can be subdivided into two classes, radioisotopes and fission reactors. Radioactive isotope power supplies have been utilized to power the lunar experiments package left by Apollo astronauts, as well as the Pioneer 10 and 11 spacecraft. In the Radioactive Thermoelectric Generators (RTG's), heat released by the isotope is converted into electrical energy by using the Seebeck thermoelectric effect known most familiarly in ordinary thermocouples. Current RTG designs could cost as little as $\$ 500$ /watt (much of this is the cost of the isotope), and are about an order of magnitude heavier, at $0.43 \mathrm{~kg} /$ watt, than lightweight solar arrays of the same power output at Earth. Thus, they offer less potential than solar arrays as a power source for electrically propelled spacecraft (see below), even neglecting costs.

Costs of RTG's are being reduced by the use of less costly isotopes. Mass reduction, however, required a conversion process with a higher conversion efficiency. Turbomachinery can be 2 to 4 times as efficient as thermoelectric converters at some penalty in reliability, which is important on long-duration missions.

The only economically feasible source of electric energy at high powers; e.g., of the order of $100 \mathrm{~kW}$, appears to be the nuclear reactor. Relatively small space power reactors have been under development for some time: the SNAP 10A, which utilized a zirconium hydride reactor powering thermoelectric cells, generated $2 \mathrm{~kW}$ in a 1965 space test, and the SNAP-8 turboelectric system, using the same reactor, has been operated on ground tests for thousands of hours at power levels up to $30 \mathrm{~kW}$. And although funding for nuclear reactor space power has been totally eliminated in the current program, any future demand for spacecraft electric power in the tens of kilowatts or higher, such as may be needed for electric propulsion (see below), will require the development of a new reactor capability. This could be either a small, compact fast-neutron reactor of the type conceived several years ago for the now-defunct 
SNAP-50 program, or, conceivably, an advanced reactor system (using thermal or fast-neutron spectra) employing thermionic diodes either in the core or powered from the core by heat pipes. Both these concepts were elements in recentlycancelled technology programs. It is worthy of note that the technology developments inherent in these programs had the potential for beneficial impact on problems of terrestrial power generation.

\section{Space Propulsion}

\section{Advanced Chemical Rockets}

One of the key elements in future use of the space shuttle is an interorbital transfer stage or, as it is more commonly known, a "space tug." The tug is needed for payloads which require trajectories or orbits other than the low Earth orbit achievable by the space shuttle orbiters, and which do not employ their own on-board propulsion system to effect transfers into the required trajectories or orbits. Although the tug is not generally applicable to most planetary missions, it can be useful for some, as well as for several Earth-orbit observation missions.

Options for obtaining a space tug capability include (1) the use of current expendable upper stages; e.g., Agena, Centaur, or Transtage, or slightly modified versions thereof; (2) development of a partially reusable interim space tug based on one of the current stages, followed later by an evolutionary process of uprating its performance and capability as necessary; or (3) direct development of a fully reusable, high-performance, cryogenic chemical rocket space tug.

It is possible that the requirement for a space tug could be delayed by continuing to operate current expendable upper stages for a period of time after the initial operational capability date of the space shuttle. The optimum space tug development path will depend on the available funding, the space shuttle availability date, and the determination of payload requirements.

On-board spacecraft propulsion is required to provide the energy for injecting scientific payloads, and in some instances landers, into desired planetary orbits. Current orbiter spacecraft propulsion systems utilize "Earth storable" propellants; i.e., they are chemicals normally liquid at Earth temperatures and pressure. Planetary spacecraft must remain in space for months or years; thus the onboard systems must exhibit long life while exposed to the space environment. Earth storable propellants would freeze in space without an active thermal control system to maintain their temperatures in the $60^{\circ}-70^{\circ} \mathrm{F}$ range.

As missions become longer and more difficult; e.g., Jupiter or Saturn orbiters, higher-performance propulsion systems will be needed to provide acceptable scientific returns. The most promising advanced propellants for future planetary missions are a class of liquid bipropellants termed "space storables." They -employ cryogenic oxidizers (liquid at $-250^{\circ}$ to $-300^{\circ} \mathrm{F}$ ) such as fluorine $\left(\mathrm{F}_{2}\right)$ or oxygen difluoride $\left(\mathrm{OF}_{2}\right)$. The fuels are either Earth storable, including monomethylhydrazine $\left(\mathrm{CH}_{3} \mathrm{NHNH}_{2}\right)$ and hydrazine $\left(\mathrm{N}_{2} \mathrm{H}_{4}\right)$ or they can also be cryogenic, such as diborane $\left(\mathrm{B}_{2} \mathrm{H}_{6}\right)$. The cryogenic nature of these chemicals makes them ideally suited to the space environment; they can be stored as long as desired with passive thermal control systems (insulation, radiation coatings, etc.). In addition, the use of fluorinated oxidizers improves the propulsion system performance by $25-30 \%$. For some of the more difficult missions this can mean an increase in useful mass in planetary orbit of as much as $60 \%$.

\section{Electric Propulsion}

A major potential performance gain for solar system exploration missions, especially for missions to comets and asteroids, can be obtained by using electric propulsion. An electric rocket's advantage over a chemical rocket is its higher effective propellant exhaust velocity*: 30,000 to 80,000 or more meters $/ \mathrm{sec}(100,000$ to $270,000 \mathrm{ft} / \mathrm{sec})$ compared with 3,000 to 4,000 meters/sec $(10,000-13,000$ $\mathrm{ft} / \mathrm{sec}$ ) for chemical rockets. Although electric propulsion devices operate at very low thrust levels, typically 0.05 newtons (10, millipounds) per kilowatt of power, they provide high total impulse; i.e., millions of newton-seconds if operated continuously for many months or even years, and their high effective exhaust velocity keeps propellant consumption low. Electric propulsion devices operate by electrically accelerating very small masses of ionized propellant vapor. The electric power needed can be obtained from solar arrays (solar-electric propulsion) or by on-board (usually nuclear) power supplies. A range of 2 to $100 \mathrm{~kW}$ of electric power is needed to provide the power required by the accelerating electrodes of an electric rocket (for comparison, the initial design output of the Skylab workshop's solar array panel was $16 \mathrm{~kW}$ ).

The need for solar illumination restricts most solar-electric missions to inner-planet ranges.

\footnotetext{
*Effective exhaust velocity (often improperly termed "specific impulse") is a direct measure of rocket propellant effectiveness; i.e., the higher the effective exhaust velocity, the less propellant is required to maintain a given thrust for a given period of time.
} 


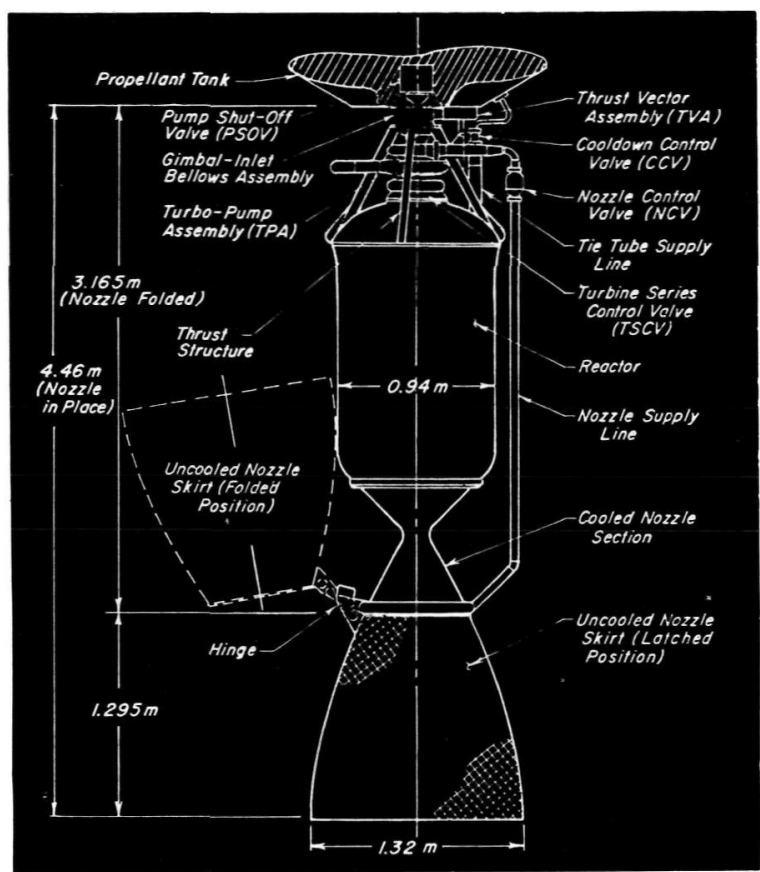

39 Solid-core nuclear rocket engine.

Nuclear power is needed for electric propulsion systems utilized on outer-planet missions. These power supplies could utilize dynamic power systems such as Brayton-cycle gas turbines or solid-state (e.g., thermionic diode) systems powered by a small nuclear reactor.

Electric propulsion devices are attractive for missions to low-gravity bodies such as comets and asteroids because their low thrust applied over a long part of the mission permits the spacecraft to approach slowly, and rendezvous by flying in formation with the small body. The spacecraft can then land if the target is solid, or at a comet, electric propulsion permits the spacecraft to maneuver extensively within its envelope to explore coma, tail, and nucleus.

The fundamental difficulty in rendezvous missions to small bodies is the large uncertainty as to their exact position. Electric propulsion can be used to change the rendezvous trajectory as the spacecraft approaches the body, and allows sufficient performance margin to ensure rendezvous.

Solar-electric missions have been studied in considerable detail to identify scientific objectives and match them to instrumentation, to evaluate alternative mission modes, define subsystem characteristics and vehicle configuration (Fig. 38) and to estimate development costs. Hardware development testing with various propellant-thruster schemes has been underway; the most recent U.S. Earth-orbit flight verification test of an electric propulsion system was SERT II in 1970. Further development work is necessary and timely to be ready for a 1979 launch for a slow flyby of Comet
Encke and for comet rendezvous missions in the early 1980's.

\section{Nuclear Propulsion}

Although NASA has recently terminated its nuclear propulsion research program (January 1973), the nuclear rocket technology reached a rather advanced state of development, and could conceivably be revived in the later part of this century should the need for high-energy missions become important. The nuclear rocket also offers attractive dual-mode capability; i.e., the reactor can be used both for direct, high-thrust rocket propulsion and also as a power source for low-thrust electric propulsion devices.

First conceived in the mid-1940's, the solid-core nuclear rocket heats a propellant fluid to high temperature by nuclear fission energy in a reactor and then expands it through a nozzle to produce thrust (Fig. 39). Because the working fluid needs no special chemical properties (ordinary rocket propellants must be flammable, for example), it is possible to use pure hydrogen as a monopropellant to achieve effective jet velocities about twice those of the best chemical bipropellants; e.g., hydrogen and oxygen. Because of its high engine mass (due to the reactor) as compared with highperformance chemical rockets, however, its best potential applications are for missions requiring very high changes in momentum; i.e., either massive payloads or large changes in payload velocity. Nuclear rockets thus offer unique trajectory opportunities not possible with either chemical or electric rockets. 


\section{Strategy \\ for Solar System Exploration}

This chapter reviews the implementation of a solar system exploration program, beginning with missions already approved and scheduled by NASA and suggesting further programs that might be valuable in studying the planets and their satellites, the interplanetary medium, and the comets and asteroids of the solar system.

By 1990 we can have completed a preliminary reconnaissance of each of the planets, many of the satellites of the planets, and some of the comets and asteroids. We can also have returned samples from several of the natural satellites, the asteroids, and perhaps some comets; and orbiters at many planets can be reporting routinely and continuously on the status of each planet's atmosphere, surface, and radiation fields - as we are now doing for Earth. A number of options for conducting a viable program of space exploration are examined.

\section{A. OBJECTIVES}

In NASA's program for the 1970's, Robert Kraemer, Director of NASA Planetary Programs, indicates that "...exploration of the terrestrial planets will continue as a strong effort, but will be balanced with a comparable investigation of the outer solar system." This rationale presumes that obtaining some data from each of the planets provides a better cross-section of the entire solar system than concentration of resources on one or two planets. It also implies a reasonable balance between expenditures on immediate missions and investment in the development of the technology which will make future missions possible.

In formulating an unmanned solar system exploration program, it is necessary to develop a progressive understanding of each target, so that proposed missions may build on results obtained from earlier ones. Generally, the sequence of exploration would start with Earth-based observation, would proceed to flyby missions, and then to considerably improved orbiter missions. Next would come the investigation of planetary atmospheric composition, structure, and dynamics by atmospheric probes, followed by landers to study geology and, where appropriate, biology. Finally, sample- return missions would be considered, to allow analysis in Earth laboratories.

As we have shown in Chapter 3, there is still much to be learned about the other bodies of the solar system. Even for Mars, which was mapped in detail by Mariner 9 in 1971-72, the total information we obtained was only slightly more than what we knew about the Moon using ground-based telescopes before the first lunar probes. For observing the surface features of the other planets, we can do little better with the largest Earth-based telescopes than we can do observing the Moon with the naked eye.

When the Mariner-Venus-Mercury mission is completed in 1974, all of the inner or terrestrial planets will have been investigated either from flyby or orbit by unmanned spacecraft. Thus, 17 years after the Soviet Sputnik opened the Space Age, man-made machines will have written the preliminary chapters to the first phase of exploration of the solar system.

The U.S. space capability has matured significantly since the first Explorer orbited the Earth in 1958. We have set men on the Moon, measured the atmosphere and ionosphere of Venus in depth, and charted Mars in detail in the visual, infrared, and ultraviolet portions of the spectrum. Our knowledge of interplanetary space has advanced spectacularly; we have learned much about the environment in which the Earth moves - the solar wind, the particles and fields, the cosmic dust, and the trapped radiation belts.

During coming decades, we will be emphasizing the exploration of the outer solar system. However, a well-integrated program also should include simultaneous investigation of the inner solar system and the interplanetary medium.

We will now consider some of the available options and the directions in which the national space program might go in the coming decades. We examine the attempt to achieve a balance between restricted resources and the selection of programs that promise the most scientific return while advancing the technology and contributing to the bank of human knowledge.

\section{The Inner Planets}

\section{Mercury}

Future missions to Mercury would be scientifically productive but they presently have less priority than those to Venus. Nevertheless, further exploration of the planet should have an important place in NASA planning. The most important areas for further exploration of Mercury are the following:

1. Detailed mapping of surface features. 
2. Detection and measurement of a possible thin atmosphere.

3. Measurement of the magnetic field and particle environment.

4. Determination of the composition of surface materials.

The first three of these areas can be accomplished by flyby and orbiter spacecraft, such as the 1973-74 Mariner Venus-Mercury mission. Some information on the surface composition could be obtained by remote sensing from such spacecraft, but more definitive results would require lander spacecraft equipped for soil sampling.

Mercury, the planet closest to the Sun, has been baked by solar radiation since the formation of our system. Knowledge of the effects of this intense radiation on the atmosphere - if any is still left-and the surface of Mercury will enhance our understanding of the processes through which the Sun's radiation influences the state of our Earth.

It is difficult to describe the most productive set of missions to Mercury until data from the 1973-1974 flight have been analyzed. However, as with any of the planets, an orbiter would yield significant data, particularly with reference to the solar wind and highly energetic charged-particle fluxes from the proximity of the Sun. A Mercury orbiter would also allow a sustained investigation of the internal structure of the planet and would contribute valuable information to the theories of evolution of the terrestrial planets. The orbiter would also provide more data for determining the shape of the Sun by an accurate study of Mercury's orbit.

\section{Venus}

The next step in our understanding of Venus involves determination of the minor constituents of the atmosphere, measurement of the composition of the clouds, and mapping of surface features.

The minor constituents of the atmosphere can be measured in part by improved ultraviolet and infrared spectrometers on flyby and orbiter spacecraft, in the regions of the atmosphere above the cloud layer. More detailed measurements, extending down to the surface, require the use of atmospheric probes, improved in sensitivity and accuracy over the early instruments used in the Russian Venera probes. For example, we would like to know if nitrogen and argon are present in Venus' atmosphere and in what amounts, since these data are very important in determining the ways in which the history and development of Venus have differed from those of the Earth. Although these gases have not yet been detected in Venus' atmosphere, the sensitivity of the measurements is not adequate to rule out amounts of these gases of the same order as those found on Earth.

The composition of the clouds of Venus is an important question related to our understanding of the physical chemistry of planetary atmospheres which can be answered only by direct measurement with an atmospheric probe. Also, a study of the optical properties of the clouds from below, as well as from above, should help in determining the factors which control the heat balance of the planet.

Because of the dense cloud cover, surface mapping of Venus is practical only with radar techniques. This could be done by a spacecraft in polar orbit about Venus; the basic method has already been demonstrated in terrestrial applications. It will be of great interest to compare the surface features of Venus with those of the Earth and Mars. We would expect the geological properties of Venus to be comparable to those of the Earth, but we do not know how the erosional properties of the hot, dense Venus atmosphere compare with those of the water and the relatively light nitrogen/ oxygen atmosphere of the Earth. Also, we do not know to what extent the high temperature of the surface of Venus modifies the geological processes in its crust. Recent radar observations of Venus (made from Earth) indicate that its surface characteristics may be vastly different than those of the Earth, but data in far greater detail are needed.

Direct sampling of the surface materials and comparison with the Earth, Moon, and Mars will be of great interest, in particular because the relative abundances of various elements may have some bearing on the cause of the apparent lack of water on Venus. Seismic activity is also important because of what it will tell us about the geologic processes in the crust of Venus, and also about the structure of the interior. For example, we would like to know whether the fact that Venus has no appreciable magnetic field is due to its lacking a molten iron core like that of the Earth, or is simply a consequence of its slow rotation rate.

\section{Mars}

Although Mariner 9 told us more about the surface and environment of Mars than any other planet except Earth, many questions remain to be answered. Perhaps the foremost of these is whether life exists on Mars. The Viking missions in 1975 will address this question. Beyond Viking, future unmanned Mars missions should seek to return surface samples to Earth, since, until men are able to land on and return from the planet, this technique may be the only hope for settling the question of life on Mars. Biologists believe that, if life existed on Mars for any considerable period, we will find either living organisms or fossil traces. 
Age-dating of these samples in Earth laboratories might settle other questions as well, such as how the volcanic structures formed and how the dry stream beds evolved. A strict quarantine must be observed with Martian samples to avoid contaminating the Earth. We need improved measurements of the atmospheric and surface compositions, which can be obtained best with lander-type spacecraft. For example, we would like to know whether water exists beneath the visible surface in the form of permafrost or hydrated minerals, and whether nitrogen and argon exist in the atmosphere in the same proportions to carbon dioxide and water as on Earth.

A lander should also emplace seismometers on Mars. By comparing its seismic activity to those of the Earth and Moon, its internal structure can be inferred. Some scientists still believe that Mars has a molten core because of the evidence of volcanic activity, and that the rotating planet should generate some measurable magnetic fields, as strongly suggested by earlier Soviet Mars spacecraft. Future missions should look for additional evidence of this kind as confirmation of this earlier exploration.

There are also many tasks yet to be addressed by orbiters. Future orbiters should certainly look carefully at the seasonal behavior of dynamic events, conduct surveys of geological processes, perform surface chemistry measurements from orbit, and monitor the poles more closely. (Although its original mission had long since been completed, Mariner 9 ran out of attitude control gas as it was completing photographic surveillance of the north polar regions.) An orbiter can best determine the answers to the anomalies of mass distribution, record other gravitational perturbations, and look for indications of a magnetic field or trapped radiation belts. These tasks can be performed with plasma probes, magnetometers, ultraviolet spectrometers, and other on-board instrumentation.

\section{The Outer Planets}

\section{Jupiter}

Among the needs for further investigation of the "big planet" are more detailed studies than those of Pioneers 10 and 11 of the structure and composition of its atmosphere, its magnetic field, trapped radiation zones, and thermal radiation output. Also of great interest are mapping of the surface features of Jupiter's satellites, search for possible atmospheres, and measurements of their surface composition.

Of the components of Jupiter' atmosphere above the cloud layer, only the minor constituents methane and ammonia are readily measure- able at present. The major constituent, molecular hydrogen, can be measured only with difficulty and poor accuracy. The next most important constituent is believed to be helium, but it is undetectable with ground-based methods, as are neon, argon, and molecular nitrogen. Definitive measurements of the atmospheric composition can be made best with atmospheric probes, although ultraviolet and infrared spectrometers on flyby or orbiter spacecraft can provide much new information. Knowledge of the hydrogen/helium ratio will advance the understanding of how Jupiter and the other outer planets were formed. If the ratio is greater than 10, the Sun's value, then Jupiter is essentially the same in composition as the original gas in the solar nebula from which the solar system was formed (since the Sun has "burned" some of its hydrogen during its lifetime). If it is appreciably less than 10 , then some hydrogen was lost in the process of forming Jupiter. Many other atmospheric constituents are likely to be found in the warmer region of the atmosphere below the cloud layer, such as water (probably more abundant than methane), hydrogen sulfide, cyanides, and others.

Since Jupiter is the only planet in the solar system other than Earth which is known to have a magnetic field, it will be of great interest to define its magnetic field and trapped radiation zones by detailed in situ measurements by space probes. It will also be useful to search for aurorae on Jupiter and to compare their morphology and intensity with those of the Earth.

The satellites of Jupiter and the other outer planets are expected to be quite different in composition and structure from our Moon. There is already evidence that some of them have water (ice or frost) on their surfaces, and water ice may be a major constituent of these satellites. Other volatile compounds, such as ammonia and various hydrocarbons, are probably also present, and it would be of obvious interest to verify this by direct sampling. Detailed imagery of the satellite surfaces will tell us something about the history of meteoric bombardment in this region of the solar system, and about geologic processes on these satellites. It will also be useful to measure the composition and total pressure of any residual atmosphere on these objects, especially Ganymede and Io, (where Pioneer 10 detected a tenuous ionosphere) as well as their interactions with the magnetosphere of Jupiter.

\section{Saturn}

The objectives in the study of Saturn's atmosphere are very similar to those for Jupiter. Present evidence indicates that the atmospheres of the two 
planets are probably quite similar in composition, but that there are significant differences in their cloud structures. However, it may be technically much easier to probe Saturn's atmosphere than Jupiter's, largely because the lower gravitational potential of Saturn makes the entry heating of the probe's heat shield much less severe.

Although Saturn has not yet been shown to have a magnetic field and trapped radiation belts, it still is important to make on-site measurements with instrumented probes. Another interesting subject is the composition of Saturn's rings. Recent radar probing has indicated the presence of solid rocks of the order of several meters in size. Only close-up imagery by space probes will give us the definite answer.

Titan, largest satellite of Saturn, is the only planetary satellite definitely known to have an appreciable atmosphere. Only its methane content is readily measurable from Earth. The presence and amounts of other, less detectable constituents are of interest, as are the total surface pressure and the presence and composition of clouds. A comparison of the surface temperature distribution of Titan with those of other, atmosphereless satellites of Saturn would shed light on the contribution of any greenhouse effect associated with Titan's atmosphere. The surface features and surface compositions of Titan and the other Saturn satellites are of interest for the same reasons given for Jupiter's moons. One special case is the satellite Iapetus, which appears to have six times the reflection on one half as the other, indicating possibly that one side is covered with ice and the other side has had the ice removed by some unknown process.

\section{Uranus and Neptune}

The objectives for the exploration of Uranus and Neptune are similar to those for Jupiter and Saturn. Because of their higher densities, we expect their atmospheric compositions to include higher proportions of heavier elements (including possibly helium) relative to hydrogen than does the Sun's. As in the case of Saturn, it will be of interest to determine the presence and strength of any magnetic fields or trapped radiation zones, and to investigate the satellites, particularly one, Triton, which is nearly the size of Saturn's Titan but has not been detected to have an atmosphere. Onsite search for an atmosphere is an obvious scientific goal.

\section{Pluto}

Little serious thought has been given to Pluto, unless flights could be adjuncts to a Grand Tour and require no special preparation. At a mean distance of 40 astronomical units from the Sun,
Pluto rides the perimeter of the solar system on the most eccentric orbit of any of the planets, and represents an extremely difficult task for any reasonable technology projections.

\section{The Asteroids and The Comets}

Because of their small sizes and great distances from Earth, very little has been done to generate images of asteroids. Even the asteroid Eros, which is more than twice as long as it is wide, is visible with Earth-based telescopes only at its closest approaches to Earth (some 22 million $\mathrm{km}$, or 14 million miles). Of the asteroids in the regular "asteroid belt," even the largest are just detectable as other than point sources in the largest Earth-based telescopes. Hence, close-up observation by space probes, and observations by the Large Space Telescope (LST) in Earth orbit, are of high priority in initial asteroid studies.

Direct sampling of one or more asteroids is also desirable. Many meteorite trajectories suggest that they are fragments of larger asteroids broken up by collisions. It would be of great interest to attempt to verify this by comparison of compositions. Also, the asteroids which never approach the Sun may contain more volatile materials than do meteorites which come close enough to impact the Earth.

Direct sampling of cometary constituents by means of space probes is the best method of determining their composition. However, ultraviolet and infrared spectroscope measurements from fly-by space probes or from near-Earth space telescopes will provide much helpful new information. On-site measurement of magnetic fields and solar wind/particle environment will help answer outstanding questions regarding the mechanism by which the so-called "ion tails" are excited. Ion tails show strong emissions of carbon monoxide and nitrogen ions which cannot be accounted for by solar ultraviolet radiation or the steadystate solar wind.

Determination of the composition of comet nuclei (dust and volatile compounds) will increase our understanding of the origin of these objects by comparison with the composition of other objects such as the asteroids and the satellites of outer planets.

4. The Sun and the Interplanetary Medium

The only part of the Sun which is directly observable is termed its "atmosphere"- constituting only about $2 \times 10^{-10}$ of its mass. In that atmosphere, we find a fascinating array of complex activities. Basic problems related to these activities, which are important to our understanding of the Sun, include the following: 
(a) Energy Transport, from the thermonuclear source at the core of the Sun to the photosphere, and uphill beyond the temperature minimum $\left(\sim 4500 \mathrm{~K}\right.$, or $\left.8000^{\circ} \mathrm{F}\right)$ into the hot chromosphere and still hotter corona $(\sim 2,000,000 \mathrm{~K})$. What is converted into high-energy radiation and charged particles? The process is analogous to using the energy of a slowly-moving battleship to propel machine-gun bullets.

(b) Solar Magnetism, its origin, evolution and distribution through the Sun and its atmosphere.

(c) Solar Activity - the physical processes underlying sunspots, flares, prominences, etc.

(d) The Solar Cycle-what causes solar activity to vary with an 11-year period?

(e) The Solar Chromosphere and its physical nature-why does it exist?

(f) The Solar Corona-How does activity near the solar surface influence the corona? What are the details of the origin of the solar wind?

(g) Abundances-What are the abundances of the various elements in the Sun? Do they differ in various parts of the Sun or features of its atmossphere?

(h) Solar Evolution-What is the history of the Sun's temperature and luminosity since its origin, and what will happen in the future?

These problems, of course, are not independent. As we have seen in Chapter 3 , we already have partial solutions to some of them. However, much more in the way of observational results is needed to fill in the details of our current general picture and to increase our understanding of these phenomena and their inter-relationships.

The Sun communicates its activities throughout the solar system by means of the solar wind-by which, as we have seen, direct effects are produced near other solar system bodies that can be detected and measured by instruments on space probes. An increased knowledge of the manner in which the effects of solar activity are propagated through the solar system is needed, as well as improved knowledge of the properties of the solar wind itself, and of the bursts of energetic particles ejected by solar flares. Also, the nature of the interaction of the solar wind with planetary magnetospheres, with comets, with planets without magnetic fields (with and without atmospheres), and with the surrounding interstellar gas need much further investigation.

These investigations can best be carried out with space probes exploring the largest possible volume of the solar system, ranging from as close to the Sun as possible out into the undisturbed interstellar medium and also out of the ecliptic plane. Many (but not all) of these objectives can be carried out on the same missions directed primarily at exploring the planets.

The interplanetary dust is also an important area of investigation for space probes. As an example, experimenters had expected that Pioneer 10 would encounter a considerable density of cosmic dust during its transit of the asteroid belt. This microscopic particulate matter should be abundant in this region because of collisions between particles of greater size. The instruments on board Pioneer did not record a significant change in density, however.

Before Pioneer, the cosmic dust in the inner solar system had only been measured between Earth and the planets Venus and Mars. Measurement of the dust profile beyond Jupiter could establish whether there is a dust belt somewhere other than in the asteroid zone, that feeds dust into the terrestrial region of the solar system.

The dust content of Saturn's rings is of great interest to scientists who want to know whether these particles are similar to those found elsewhere in interplanetary space. These questions can only be answered with spacecraft instrumentation.

\section{Manned Planetary Exploration}

In the past, there have been numerous discussions supporting the idea that the next major national space goal should be the placing of men on a planet, presumably Mars, since the other terrestrial planets appear to be even more inhospitable than our Moon.

In order to maintain the option of eventual manned exploration of the planets, several groups have recommended that NASA undertake such biomedical programs as exposing man to space conditions for long periods (100 to 200 days) in Earth orbit to determine whether he is qualified to undertake planetary missions that involve round trips of about 700 days.

There is little reason to believe that manned expeditions to Mercury or Venus would be practical. Both planets would require an extraordinary technological effort just to keep men alive. The weight and complexity of the survival equipment would make the cost prohibitive, especially in light of the extremely hostile environment. It is likely that, at least for the remainder of this century, unmanned spacecraft will continue to carry the full burden of planetary exploration.

Inevitably, when considering future missions to Mars, the question of landing men on the planet must arise. Such a project would be much more expensive than Project Apollo, and the flight would require at least two years for completion. It seems unlikely that, in this century, we will undertake to transport men to Mars and to land 
them on the surface of that planet.

In July 1968, the Space Science Board of the National Academy of Sciences said: "In the face of a limited space budget, we favor reallocation to the unmanned exploration of the planets those resources directed to efforts preparatory to a manned planetary program. The rapid development of technology suggests that fully automated systems of substantial complexity will be available for planetary exploration and that this technology should be capable of answering the major scientific questions that we can now pose about the planets.

"While at some time in the future it may be in the national interest to undertake manned missions to the planets, we do not believe man is essential for scientific planetary investigation at this stage. Therefore we recommend that those resources presently intended for support of manned planetary programs be reallocated to programs for instrumented investigation of the planets.".

\section{B. MISSION MODES AND OPPORTUNITIES}

The dynamic relationships existing among bodies of the solar system present a rich variety of opportunities for missions in the 1973-1990 time period. One missed opportunity was the Grand Tour, shown in Fig. 40, a concept made possible by a rare alignment of the outer planets in the late 1970's which will not reoccur for another 179 years. This mission will not be flown for budgetary reasons and because scientists were not in agreement about various payload requirements. Also, its value was questioned, as compared with alternative means of exploring the outer solar system. Instead, Mariner flybys of Jupiter and Saturn will be undertaken in 1977, as described later. There will, furthermore, be many more-frequent opportunities for multi-planet flybys of some of the outer planets and visits to asteroids and comets, so that cancellation of the Grand Tour has not been catastrophic to the program.

In this section, we outline first the existing programs (that is, those which have already been approved) to exploit some solar-system exploration opportunities, and then consider some of the future possibilities.

\section{Approved Missions}

\section{Mariner Venus-Mercury 1973}

A Mariner-type spacecraft (Fig. 25) was launched on November 3, 1973, on a gravity-assisted flyby of Venus and Mercury. This mission uses the gravitational field of one planet (Venus) to deflect the spacecraft trajectory in such a way as

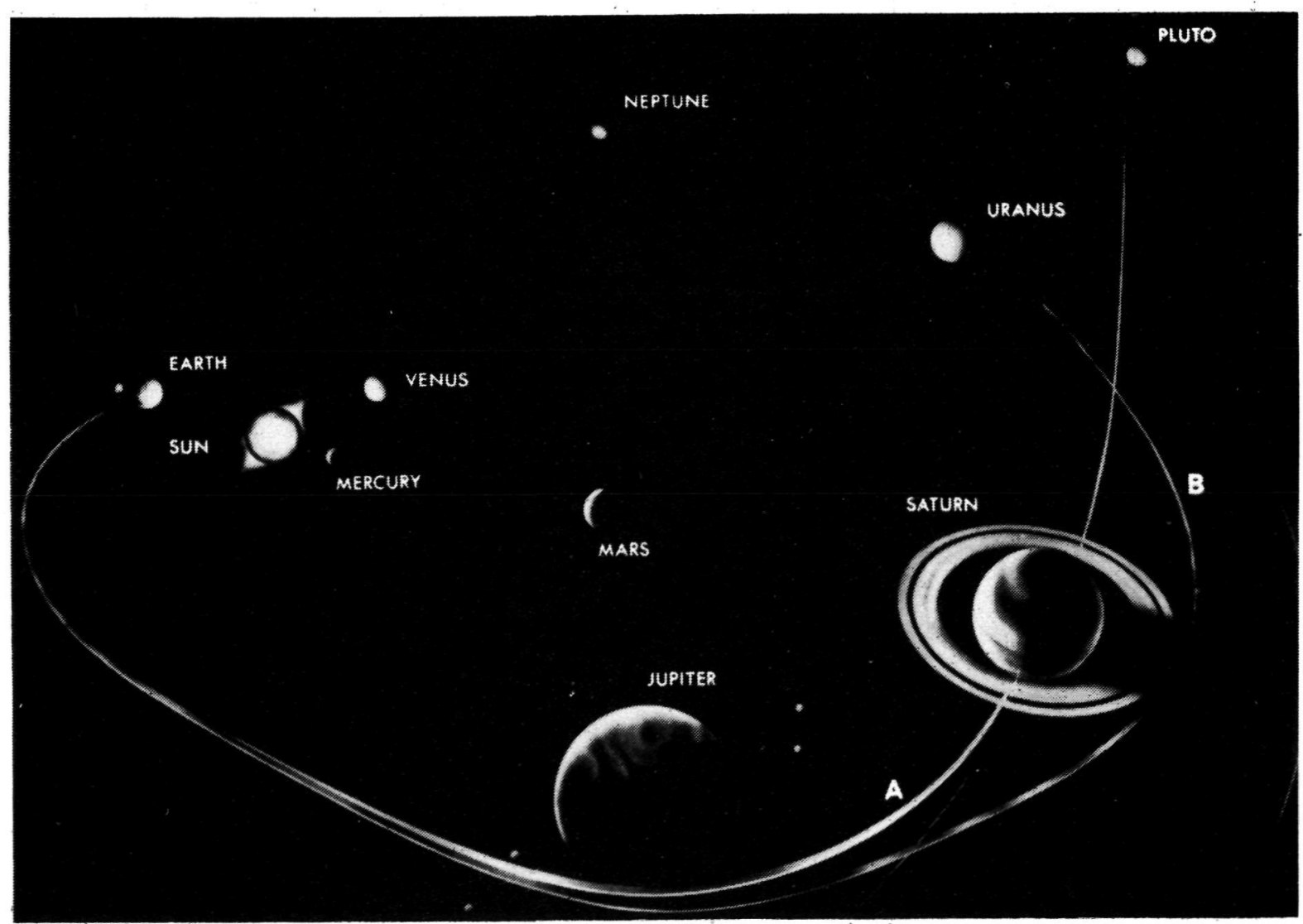

40 Grand Tour gravity-assist opportunities in late 1970's. Trajectory A shows how a single spacecraft could flyby Jupiter, Saturn, and Pluto; in B, Jupiter, Uranus, and Neptune. 
41 Mariner

Venus-Mercury

flight trajectory.

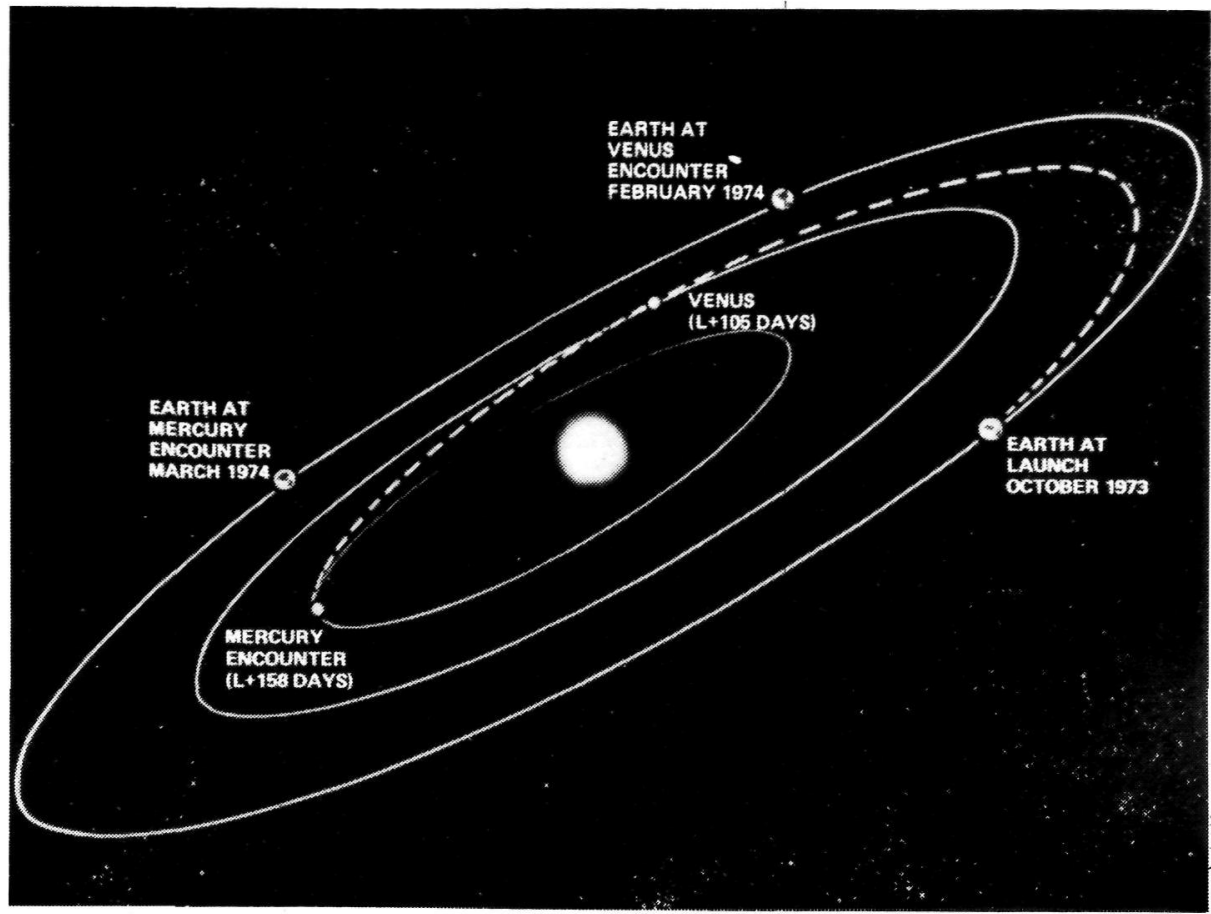

to impel the vehicle on to another planet (Mercury) without expending additional propulsive energy, a technique which conserves flight time and saves about $50 \%$ of the energy otherwise required at launch. It was used earlier on Pioneer 10, which was sent completely out of the solar system by utilizing Jupiter's gravitational field.

The spacecraft arrived at Venus February 5, 1974, and reaches Mercury on March 29, 1974 (see Fig. 41). The scientific objectives of the mission largely concern Mercury, which is so close to the Sun and is seen from Earth at such a small angle from the solar body that we have never had a clear view of the planet. The experimenters expect to obtain photographs of Mercury's surface with resolutions down to about 9 meters ( 30 feet). They hope to take pictures of most of the illuminated hemisphere with a quality approximating those obtained by the wide-angle camera on the most recent (Mariner 9) mission to Mars, or approximately the resolution obtained in Earthbased telescope photographs of the Moon. The objectives of other experiments were discussed earlier.

\section{Viking Mars 1975}

The 1975 Viking project has been designed to explore the possibility of life, perhaps in microorganism form, on Mars. A positive result would certainly stimulate enormous interest in further research to investigate the detailed characteristics of Martian life. On the other hand, a negative result (which is more probable) would not necessarily demonstrate the absence of life. It might mean that we had looked in the wrong place or that the conditions provided in our experiments were for some reason not conducive to the growth of Martian organisms. Or it might merely imply that only manned exploration can provide the versatility needed to obtain a reliable answer.

Four landing sites for Viking have been designated (Fig. 42) - two prime and two backupall with strong emphasis on the search for life and its corollary: traces of surface water, either present or past. One prime site is $19.5^{\circ} \mathrm{N}, 34^{\circ} \mathrm{W}$, in a region known as Chryse. Several channel-like features, possibly the result of past water erosion, end in the region, which may indeed have been a depository for ancient Martian rivers. Because of the profound importance of water for terrestrial life, this Chryse region-relatively smooth compared to the rugged topography of the channels themselves-is an outstanding candidate for a biologically oriented Viking lander to investigate (see Chapter 4 for spacecraft details).

The second prime landing site is in the area Cydonia, north and east of Chryse, at $44.3^{\circ} \mathrm{N}$, $10^{\circ} \mathrm{W}$. It is hidden in winter by the northern polar hood, a dense cloud deck that forms over the polar region and extends to mid-latitudes of the planet. Mariner 9 observations suggest that the clouds at the latitude of Cydonia are composed of water ice. The winter ice cap, made of condensed carbon aioxide, does not, however, reach this far south, as Mariner 9 pictures have shown. The Martian atmosphere exhibits variations in the amount of 
water vapor that it contains and also in where that water vapor is to be found. It is believed that, at the season of the Viking landings, Cydonia will be a particularly favorable location for the occurrence of water vapor and hence for conduct of biological studies.

\section{Pioneer Jupiter 1973}

NASA's Deep Space Net is currently tracking two Pioneer spin-stabilized spacecraft (Fig. 23). Pioneer 10, which passed Jupiter on December 3, 1973 , was the first probe to penetrate beyond Mars' orbit. Pioneer 11 will reach Jupiter in November 1974. The primary objectives of these missions have been discussed earlier; a secondary objective is to develop advanced technology and operational capability for long-duration missions
5, 1973, and will pass Jupiter at closest approach in December 1974.

Of particular interest has been the Pioneer 10 and 11 experience in the asteroid belt that lies beyond Mars' orbit. The spacecraft were not impacted by any potentially catastrophic particles. The number of one-micron particles (a micron is a millionth of a meter) decreased with solar distance throughout the flight; 10 micron fragments remained essentially constant through and beyond the belt. A slight increase was recorded for particles measuring 0.1 to $1 \mathrm{~mm}$ ( 4 to 40 thousandths of an inch) passing close to the spacecraft, reaching a maximum number near the middle of the belt.

Whereas Pioneer 10 passed close to Jupiter's

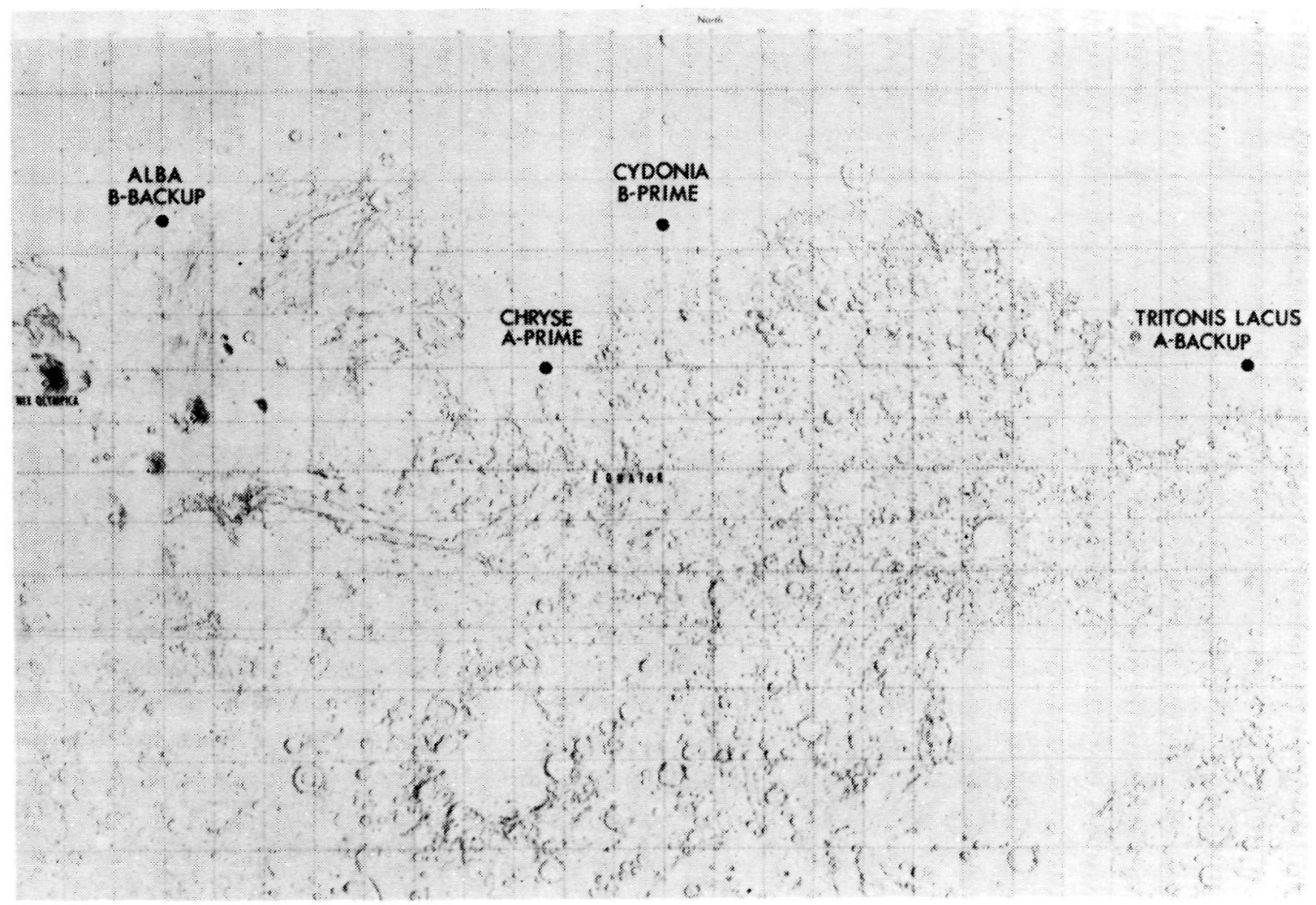

42 Prime and backup landing sites for Viking spacecraft.

to the other outer planets. Pioneer 10 penetrated the massive Jupiter radiation belt, providing photos of better quality than those of Earth-bound instruments and quantities of other data described earlier.

Pioneer 10 was launched on March 3, 1972, by an Atlas-Centaur vehicle with a solid-propellant third stage. After 19 months (Fig. 43), it passed Jupiter at a distance of approximately $130,000 \mathrm{~km}$ (81,000 miles). Pioneer 11 was launched on April equatorial plane, the encounter conditions for $\mathrm{Pi}$ oneer 11 have not yet been determined. Although Pioneer 11 may be targeted to fly over a pole of the planet, it is more likely to be guided so as to make another equatorial passage, taking it on to Saturn. An out-of-the-ecliptic trajectory carrying it over the Sun is another possibility having great scientific interest. Pioneer 10 and, possibly, 11, will leave the solar system, making them the first man-made objects to do so. 


\section{Mariner Jupiter-Saturn 1977}

A rare opportunity exists in the latter years of the present decade to fly unmanned spacecraft to several of the outer planets with dramatic savings in launch energy and mission time by use of the gravity-assist technique as discussed in connection with the Mariner Venus-Mercury mission. The only presently approved future mission of this type is a 1977 launch to Jupiter and Saturn. Titan IIICentaur vehicles with solid-propellant fourth stages will boost two $750-\mathrm{kg}(1650-\mathrm{lb})$ spacecraft from Cape Canaveral sometime during a launch period extending from mid-August to mid-September 1977. Arrival at Jupiter is tentatively scheduled between April 2 and May 15, 1979.

Accurate navigational techniques are required to enable the spacecraft to react to the massive gravitational field of Jupiter so as to acquire the
400 to 500 days before closest approach. The other on-board sensors will not respond until perhaps 40 days before arrival, when infrared and ultraviolet spectra will be acquired and a photometer and television camera will begin observations. A magnetometer and plasma probe may measure waves and particles which are ejected from the Joviar magnetosheath and the bow shock wave that engulfs the magnetosphere, in a manner similar to that of Earth.

As the spacecraft nears the planets, global coverage with imaging instruments at increasing resolution will be possible. Starting approximately 5 days before closest approach to Jupiter and Saturn the television subsystem will begin to take high resolution pictures of selected atmospheric features such as the mysterious Red Spot. Encounters with the planets' satellites will occur

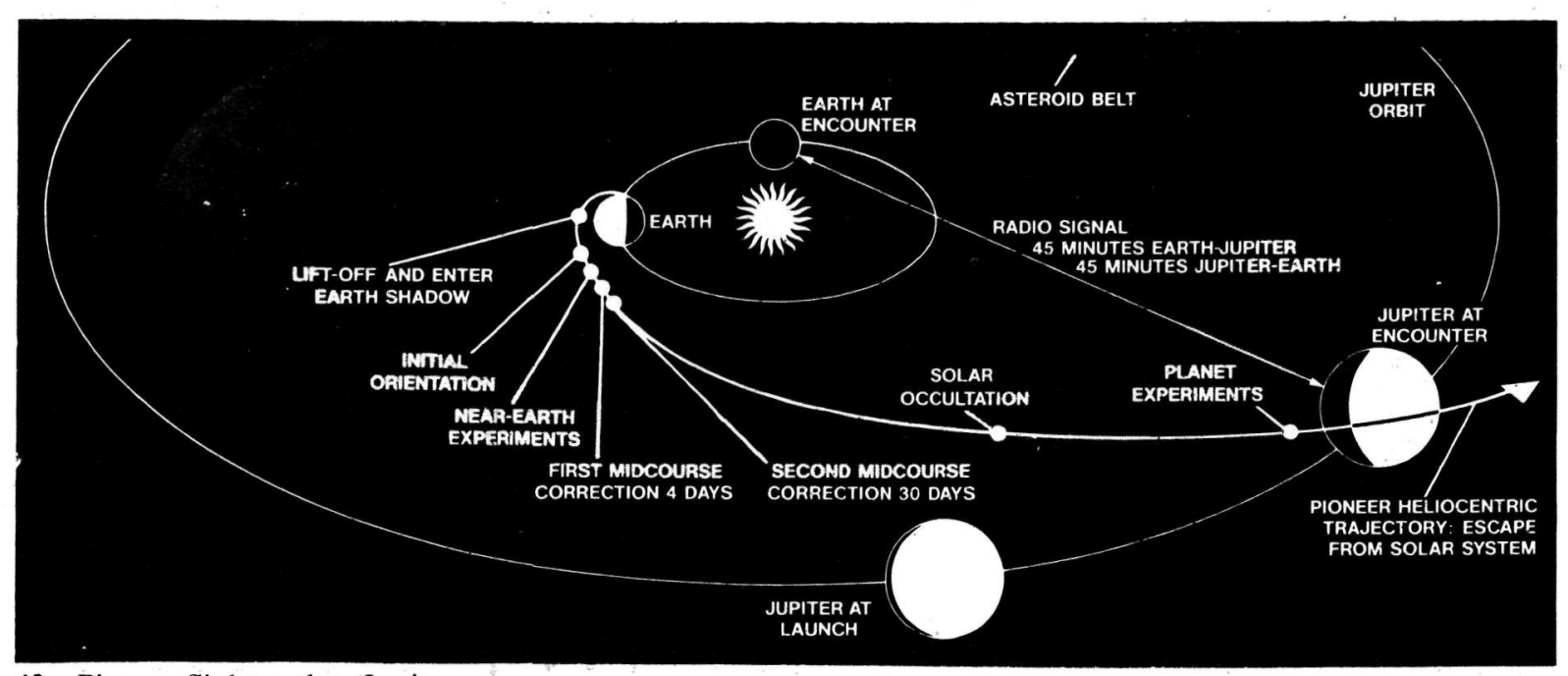

43 Pioneer flight path to Jupiter.

necessary increase in velocity to reach Saturn between January 16 and April 21, 1981. Jupiter, in a sense, acts as the last stage of the launch vehicle. The current plan is to launch two spacecraft on the 1977 mission. Cruise science data from each of the two spacecraft will be received approximately $70 \%$ of the time to obtain valuable information on the interplanetary medium. Activities during encounter, however, will be significantly different, and will occur much more rapidly than during the cruise phase. It is necessary to reprogram the spacecraft experiments for encounter operations. These are further complicated, of course, by the long signal turn-around time (2.7 hours at Saturn).

Well before reaching each of the two planets, the spacecraft instruments will sense their presence. Jupiter's radio emissions will be measured at much greater strength than at Earth as early as relatively near the planets, with Io being passed a few hours after the closest approach to Jupiter, and Titan 20 hours before the flyby of Saturn, when the spacecraft is still at a distance of about $180,000 \mathrm{~km}(112,000$ miles) from the planet.

The encounter trajectories at Jupiter and Saturn are only tentative at this time; firm selections will be made later. The options on the approach to Jupiter, however, are relatively restricted, since the number of possible trajectories which permit the spacecraft successfully to reach Saturn are limited. The encounter at Jupiter will be nearly equatorial, at a closely regulated distance, based on Pioneer experience with the Jupiter radiation belts.

At Saturn, the flyby geometry will depend in part on the hazards posed by the planet's ring structure and possible radiation environment. Scientists will want a good view of the rings with- 
out endangering the spacecraft. It may also be desirable to fly behind them for occultation purposes, thereby aiding in the determination of the density, material, and structure of these beautiful formations. It is-possible that Saturn may also have a magnetic field, so it is important again to infer itș existence by measuring a possible bow shock wave, magnetosheath, and magnetosphere with the plasma (proton and electron) and magnetometer instruments.

Saturn has ten satellites, and the 1977 mission could approach as many as four or five of them. Of these, Titan is the most interesting, since it has a relatively warm and dense atmosphere and could conceivably be the site of pre-biological activity. The spacecraft could be aimed to pass Saturn's south pole and thus to allow observations of many latitudes of the planet.

After closest approach, both spacecraft will move to the dark side of the planets. Occultation of the radio signals will reveal much about the atmospheres, while spectral instruments gather data that should prove useful in attempting to resolve the apparent ariomaly involved in surplus energy generation. This phenomenon has been shown to exist at Jupiter, and some investigators believe that it also exists at Saturn, but to a lesser degree. The spacecraft may then be targeted to pass out of the solar system.

The scientific objectives of the Mariner JupiterSaturn mission are much more ambitious than those for missions to the inner planets, largely because of the number of bodies that can be investigated. Although a big step beyond past accomplishments in planetary exploration, the 1977 mission is at best preliminary in any program to explore the outer solar system. Those remote planets are difficult to reach, involve extended flight through hazardous regions, and are only periodically available to our spacecraft. Details of a comprehensive outer planet program will flow logi- cally from the results of the preliminary missions.

Helios: 74,77

Helios-a joint U.S./West German̈ space project-will send two unmanned spacecraft (Fig. 35) into orbit about the Sun, the first scheduled for launch in September 1974, the second in Auguist $197 \underset{6}{ }$.

The primary objective of the mission (Fig. 44) is to investigate the properties and processes of the interplanetary medium in the vicinity of the Sun. and the effect of the solar body on this region. This project will-provide, new understanding of phenomena such as the solar wind, interplanetary magnetic and electric fields; cosmic rays, and cos:mic dust. Together with the Mariner Venus: Mercury, this mission will provide information about solar flare-ejected shock waves in the inner solar system.

The first Helios mission (Fig. 44) will reach a perihelion (closest approach) of 0.3 astronomical unit (approximately 45 million $\mathrm{km}$, or " 28 million miles) from the Sun and an aphelion of 1 astronomical unit, with an orbital period about the Sun of 180 days. At first perihelion passage, the period of maximum interest to scientists, it will be about 90 days into the mission. Helios will then swing on around the Sun, with the first solar occultation and radio blackout occuring at about 120 days and lasting for approximately 20 days. After 180 days, the spacecraft will have completed one revolution and returned to Earth's orbit, although our planet will have moved to the opposite side of the Sun at the time. At the end of the second complete orbit, the spacecraft will again approach within a few million kilometers of Earth near the point of original launch.

The second Helios spacecraft will be launched in August 1976 with a target perihelion of about 0.2 astronomical unit.

Germany will provide the spacecraft and the

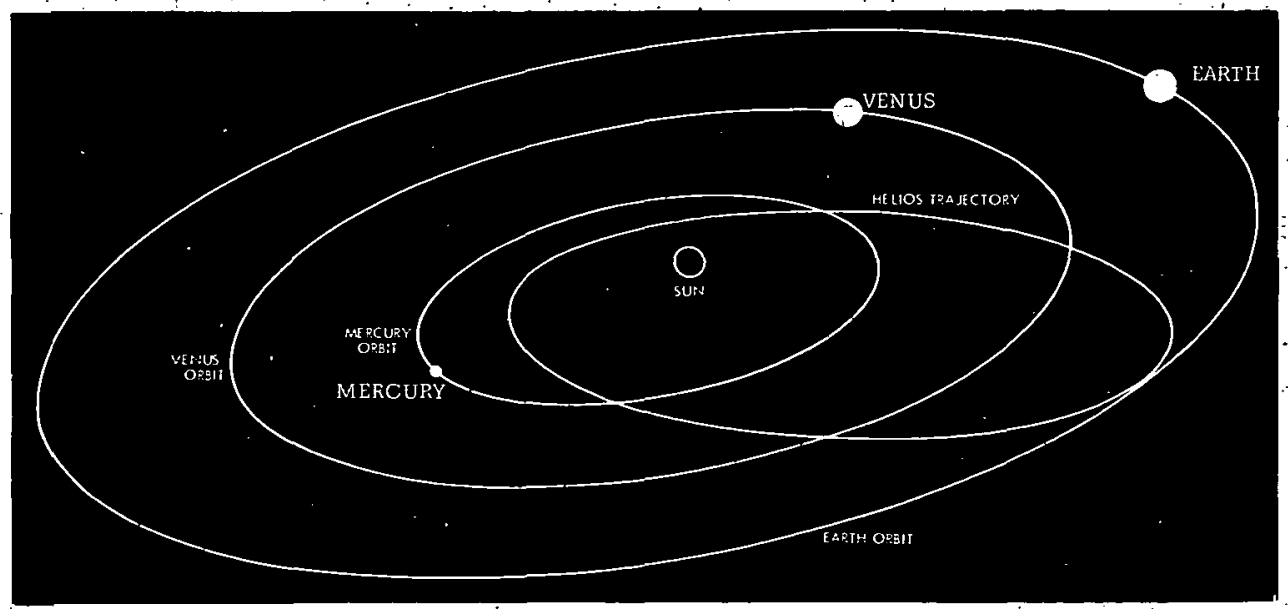

44 The Helios mission will take the spacecraft within. about 28 million -miles of the Sun, the closest approach to the solar body by a man-made vehicle. 
instrumentation for 7 of the 10 on-board experiments, and will also conduct mission operations. The U.S. will provide space simulation for final spacecraft tests, two Titan III-Centaur launch vehicles, the facilities at Cape Canaveral, three onboard instrumented experiments (and two experiments using the radio data), and the tracking capabilities of the Deep Space Net, including communication with the spacecraft, orbit determination, data accumulation, sending of commands, and position monitoring.

\section{Interplanetary' Samplers}

Several of the projects described earlier in this section will, in addition to their individual objectives, work in concert to provide an unprecedented opportunity to obtain broad-based sampling of interplanetary particles and field data to study propagation mechanisms and disturbances in the solar wind, the spatial characteristics of the Sun's heliosphere, and the effect of the solar plasma on the interstellar medium and galactic cosmic ray flux entering the solar system. This opportunity stems from the simultaneous presence of similarly instrumented spacecraft near the orbit of Mercury, in Earth orbit (Pioneers 6-9), just beyond Jupiter (Pioneer 10), and at approximately $31 / 2$ astronomical units (Pioneer 11).

\section{Opportunities in the Future}

Earth Orbit. The Apollo Telescope Mount (ATM) aboard the Skylab space station has provided new data on the Sun of unprecedented quantity and quality. Preliminary evaluation of this wealth of data already permits us to draw plans for future experiments and more advanced solar observations from space. Such observations will be prime candidates for early missions of the space shuttle, particularly those utilizing the spacelab.

Venus. As of summer 1973, no missions to Venus had been authorized beyond Mariner 10. However, high on the list of priorities is a dual-spacecraft Pioneer mission, tentatively scheduled for a 1978 launch. One of the spacecraft would be an orbiter instrumented to conduct several of the experiments discussed here. The other would carry four atmospheric entry probes, which would separate to penetrate the atmosphere and perform experiments down to the surface.

As with Mercury, orbiters also would provide the most promising future missions to Venus, permitting full surface coverage over a lifetime of at least an Earth year. The experiments should include topographical studies using radar (which can also penetrate beneath the surface), the probing of the upper and middle atmospheres with infrared scanners to investigate the thermal balance, and instruments to study the planet's interaction with the solar wind. Radar mapping from Earth is limited in that high-resolution coverage can be obtained on only one side of the planet. Spacecraft radar equipment would provide planetwide scanning from orbit.

Ultraviolet scanning of the upper atmosphere and limb (edge of the planet as seen 'from the spacecraft) could provide a look at the atmospheric constituents and some insight into the planet's atmospheric evolution. Scientists do not fully understand how Venus' atmosphere evolved to its present state while the planet is so like Earth in many other respects.

Orbiting vehicles would carry entry and landing instrument probes for measuring such factors as ion species in the ionosphere, and for making observations of the chemical composition of the atmosphere and surface.

Mars. Until men explore Mars, which is not likely to occur before the next century, unmanned landers and rovers will probably carry out the principal search for life. However, unless the first attempts find organic traces, it will probably be necessary to search in many regions of the planet.

In order to improve the range of exploration with a minimal number of missions, remotely controlled unmanned roving vehicles will be necessary. A Mars rover could traverse large regions remote from the spacecraft landing site and make many spectral and environmental measurements, analyze soil samples for biological and mineralogical content, and even look at near-surface atmospheric conditions.

It is also important that more extensive exploration of Mars be attempted from orbit. Mariner 9 spent only half a Martian year sending back information from its orbit around the planet. For comprehensive coverage, a spacecraft should be in orbit and active for at least two Martian years (nearly four Earth years) in order to record dynamic seasonal changes and other alterations in the surface environment. Much more extensive photography of uniformly high quality is needed, since the Mariner pictures, although detailed in coverage, vary considerably in quality and resolution. More conclusive information is also needed on the circulation of the atmosphere and on the alternating deposition and sublimation of an appreciable portion of the atmosphere at the poles. This periodic behavior is thought to be an important factor affecting Mars' weather, which at times can be very violent, (e.g., the great dust storm observed by Mariner 9, which probably involved $320 \mathrm{~km} / \mathrm{hr}$ (200 $\mathrm{mph}$ ) winds).

Jupiter. We have looked at the current Pioneer missions to Jupiter and the scheduled 1977 Mari- 
ner launches. Whereas Pioneer primarily investigated the energetic particles and fields, the Mariner vehicle will carry an additional complement of instruments to measure the infrared, ultraviolet, and visible-light spectra for extensive studies of the atmospheres and satellite surfaces. 1977 is probably too early for successful design of a probe that could enter and survive Jupiter's atmosphere. Factors such as the enormous mass of Jupiter and the resultant very high entry velocity make such a design extremely complex. However, even if an atmospheric probe survives the enormous heating due to entering the dense atmosphere, practical design considerations and the enormous increase of temperature and pressure limit the depth from which they will return data to approximately 100 times the Earth's surface pressure.

The year 1981 seems highly desirable for a Jupiter orbiter. Following the Mariner flyby encounter by just two years, this period is also exceptionally good in terms of launch energy considerations. Flight time to Jupiter is two years for this missiona slower transit than for the 1977 Mariner Jupiter/ Saturn flights, so that less reduction of spacecraft velocity is required to enable it to orbit the planet.

One proposed Jupiter orbiter would circle the planet once each 35 days, as compared to the 12 hours of Mariner 9 at Mars. The orbit would be very eccentric. About $90 \%$ of the time, the spacecraft would be over $1,600,000 \mathrm{~km}(1,000,000$ miles) from the planet, sometimes as far as $3,200,000 \mathrm{~km}(2,000,000$ miles). But for a brief period, it would approach within approximately $160,000 \mathrm{~km}(100,000$ miles $)$ of the "surface".

The orbiter would make the equivalent of 10 flybys in an Earth year, enough to provide good mapping of the bow shock, magnetosheath, magnetopause, energetic particles, trapped radiation, and magnetic belts within the magnetosphere. The cloudy atmosphere could also be inspected in detail. The eccentric orbit would also allow the spacecraft to encounter many of Jupiter's satellites.

Ideally, two spacecraft would be in orbit, one in the equatorial plane, the other steeply inclined and permitting measurements at high latitudes. The satellites are all in the equatorial plane and a spacecraft in proper orbit could encounter Io, Europa, Ganymede, and Callisto at least twice each in a year's time, perhaps using the gravityassist technique.

Saturn. The best opportunity for launching a Saturn orbiter seems to be 1985 , although additional energy would be needed to augment the Titan-Centaur capability in order to fly a Marinertype spacecraft. The space shuttle should be fully operational during the 1980's and, with the addition of adequate upper staging of electric propulsion, should be capable of Saturn orbiter missions.

Uranus and Neptune. During the coming period of outer-planet opportunity, flights to Uranus and Neptune might be attempted. Flight times vary, depending upon launch and propulsive capabilities. Presently, Uranus would require 6 to 10 years of flight with gravity assist, Neptune 10 to 16 years. Atmospheric entry missions to these planets would be simpler than in the case of Jupiter and Saturn, particularly in reference to entry velocity.

Asteroids. Several types of missions to asteroids are possible. The simplest mission involves a fast ballistic flyby (controlled by gravity alone as in the flight of a projectile), which restricts the types of experiments that can be performed. A slow flyby offers a much more extensive set of experimental options. It would require either a ballistic approach with strong braking capability near the target, or some form of propulsion (perhaps solar electric) which provides low levels of thrust for long periods of time.

The most scientifically desirable type of mission involves rendezvous with an asteroid, using a combination of retrorockets or other braking techniques to bring the relative velocity essentially to zero at encounter. By flying alongside an asteroid, it becomes possible to study the body for extend: ed periods, and even to land, conduct surface experiments, and return samples to Earth. A rendezvous capability would also allow the spacecraft to move about in relation to the asteroid while conducting experiments.

The type of mission would largely control the onboard experiments. The fast flyby would be capa: ble of simple visual imaging. Through higherresolution imaging, the slow flyby would allow the gathering of more definitive information on size, albedo (light reflectivity), surface homogeneity, crater density, and rate of rotation; it perhaps would permit measurement of the asteroid mass by observing trajectory deflection.

Although there are as yet no approved missions to asteroids, several programs have been proposed. A number of such missions could be undertaken, perhaps with emphasis on two rendezvous launches in 1989. These missions could use either spinning or attitude-stabilized spacecraft, but should be considered only after missions to the planets and comets. One possibility for exploring asteroids without dedicating a costly mission solely to such a project, which might be of questionable value, would be an asteroid visit en route to one of the outer planets or to a comet. 
Comets. Because of their scientific interest, missions to comets should carry strong priority. They could utilize either the fast or slow flyby, or the rendezvous flight plan. Instrumentation should identify the gases, density, distribution; the composition of charged particles, and interaction with the solar plasma, including identification of a shock front. Missions should determine whether a nucleus exists and, if so, its nature and composition.

The slow flyby permits more time to measure variations as a function of position and provides more spatial resolution, improved imaging, and angular rates. The rendezvous, as with exploration of asteroids, provides the most flexibility, including movement to several sides of the comet and time to make measurements as a function of distance from the Sun. It also is possible to fly multiple missions, given the capability of restarting the spacecraft rocket engines, in which one or more asteroids can be visited before or after a comet encounter.

In order to rendezvous with a comet; some form of terminal guidance will be required. As the comet approaches the Sun, it may experience considerable thrust from outgassing in the nucleus, thus making its trajectory something other than purely gravitational and not fully predictable. The spacecraft guidance system must be able to compensate for these variations in order to achieve either a close flyby or a rendezvous. Complex interactions with solar flare-ejected shock waves-as in the case of Comet Giacobini-Zinner in Auguist 1972-may also take place. Valuable information corcerned with the dissociation and ionization of cometary material-which may cause significant brightness changes-could be assessed with close flybys.

As with asteroids, there are no approved missions to comets, although several have been proposed. A mission to Comet Encke could be launched in 1979, with a slow flyby encounter in December 1980. A rendezvous with Encke could be launched in 1981 or 1982 , during the next visit of the comet to the inner solar system. Comet Whipple also is proposed as a target, with two or more asteroids to be encountered on the same mission. An encounter with Halley's Comet, which appears every 76 years, has been suggested for its next appearance in 1986. This would be a difficult mission, since the comet will be far from Earth and moving very fast. Work on this mission would have to start soon, perhaps including development of an advanced solar electric propulsion system and new instrumentation.

Modified Explorer and Helios spacecraft both have been proposed for fast comet flyby missions. 55
A solar electric system for a Mariner spacecraft is alco under study for use in various types of cometary rendezvous missions.

\section{Technological Constraints}

The first fifteen years of space technology and the intensive and directed Apollo development have created a broad plateau of technical achievement in those disciplines required for spacecraft. Upon examining the types of missions contemplated for the 1980's and reviewing the implementation of missions underway and soon to be developed, it becomies clear that no big, dramatic breakthroughs are required in order to complete a preliminary reconnaissance of the entire solar system in the next two decades-no Manhattan Projects or Apollo programs.

The-technical theme of the next period will be evolutionary progress as we adapt, refine, improve, and extend our gains.

New developments are, of course, required, some of them major: solar electric propulsion, atmospheric entry and high temperature technology for probes and landers, long life electronic circuits and electromechanical devices, and automated instruments. These developments are feasible. Some have long lead times which must be taken into account in program planning.

Large distances characterize the solar system: 5 astronomical units to Jupiter; 10 to Saturn; 20 to 40 to Uranus, Neptune and Pluto. This vastness implies very long mission durations: two to four years to-Jupiter and Saturn, eight to twelve years to the outermost planets if boosted by a Jupiter flyby or with the use of advanced propulsion methods. Such missions demand the utmost of sytem reliability and long-life components.

Such great distances also result in long communication delays, a concept difficult to adjust to when accustomed to "instant" communication on Earth. It takes forty minutes to send a command to a spacecraft at Jupiter and forty more minutes before an acknowledging signal can be received at Earth. This means, of course, that maneuvers requiring fast reaction must be made automatic and that fault detection and compensation must be built in.

Remoteness from the Sun poses other problems. At Jupiter, solar heat and radiation intensity is only $4 \%$ that received at Earth. At these distances, and certainly beyond, solar arrays are not practical sources of energy, nor can the Sun's rays be depended upon for heat. Of course, quite the opposite is true near the orbit of Mercury. Here the sevenfold increase in solar radiation dictates a design that must avoid absorbing excessive heat if it is to survive. 
The immediate environment of each planet, of course, affects design in a major way. Radiation fields at Jupiter, although apparently not prohibitively damaging to Pioneer 10 , may prove to be of overwhelming intensity for some contemplated measurements. Temperatures at the surface of Venus and Mercury are hundreds of degrees higher than our common design levels, and atmospheric pressures on the larger planets rival those of our deepest oceans.

All of these factors and others prove challenging, but not overwhelming, as evidenced by the missions described previously. As this is written, the marvelous Mariner 9 photographs of the Martian surface have "obsoleted several generations of textbooks on Mars," a Pioneer spacecraft has flown by Jupiter, and Mariner is approaching Mercury. These missions have been designed to avoid or surmount the problem areas of interplanetary travels, and appear to be fulfilling their objectives.

Probe missions rather than landers are the key elements in the early phases of outer-planet exploration, since it is believed that these gas giants may have no discernible surface. The objective of a probe will be to survive to a depth where the pressure may be from 10 to 1000 Earth atmospheres (equivalent to ocean pressures at 9,000 meters, or 30,000 feet) without burning up or being crushed. During descent the probe would make in situ measurements of the composition and structure of the atmosphere.

The principal new technology development required for planetary probe missions would be for heat shield testing. Existing arcjet facilities plus banks of radiating lamps to simulate the environment cost on the order of a few million dollars. But intensities must be considerably greater than. current capabilities. A fairly large capital investment might therefore be required.

Materials technology and deployment technology planned for the 1978 Venus probe can be expected to evolve into a probe which could survive for 30 minutes while descending through the atmosphere of Saturn or Uranus to either crush depth, as in a submarine's descent, or until its radio transmission is so attenuated by atmospheric absorption that no signal can emerge.

With regard to Venus itself, the solar radiation intensity is only about twice that experienced at Earth, and the planet has no apparent magnetic fields or radiation belts. Thus, there are no imposing probelms for Venus orbiters. Techniques for furling and deploying larger antennas which might be needed for radar mappers are surely within reach. The major problem which affects probes and landers at Venus is the density of its atmosphere and the extremely high surface temperatures. The latter condition is formidable. Designing instruments, data systems and radio links capable of surviving trips to the surface can be done with current technology, but survival and operation on the surface for extended periods will take a major development effort.

Of all the planets, Mars imposes the fewest problems. All of the technology used in lunar exploration (if we include the Russian efforts along with our own) is applicable to Mars, subject only to size, weight and communications constraints. Automated landing, roving and even return of surface samples have been demonstrated on the Moon, and thus pose no major technological obstacles. The only basic difference between the Moon and Mars in this respect is the need for aerodynamic braking in Mars' thin atmosphere, in addition, of course, to the already known problems raised by Mars' much greater distance and more difficult astrodynamic navigation requirements.

\section{Economic Constraints}

The budgetary constraint is almost always the primary limitation on any program. In most high technology projects, it is sometimes possible to allow performance specifications and schedules to "slip" in order to keep the budget in line. This is far more difficult to accomplish, however, for solar system exploration, because:

(1) The schedule is often dictated by orbital dynamics; for example, the opportunity for an outer-planet Grand Tour comes up only once each 178 years.

(2) The budget line item time duration is: excessive compared to most other governmentfunded programs; for example, the Mariner to Jupiter and Saturn, scheduled for launch in 1977, became a budget line item in 1972, and the mission will not be completed until perhaps 1982 . Further, the research programs on which the 1971 "go-ahead" decision was based predated the actual project initiation by a number of years. The total time-line for a given solar-system exploration project might therefore run as high as 15 years from plan to completion.

(3) Performance specifications are, to a great extent, inflexible because of the specific needs of trajectory dynamics and the limitations on launchvehicle capability. Also, a major component failure or an inaccurate maneuver such as a midcourse correction anytime during periods measured in years can wipe out a whole program.

As a consequence of these factors, only very limited programmatic changes are possible, and therefore, once a solar system exploration project has been initiated, a budget reduction could 


\begin{tabular}{|c|c|c|c|c|c|c|}
\hline BUDGET PIAN & 1973 & 1974 & 1975 & 1976 & 1977 & 1978 \\
\hline Aeronautics. & $\$ 15 \mathrm{l}$ & $\$ 171$ & $\$ 159$ & $\$ 142$ & $\$ 128$ & $\$ 117$ \\
\hline Space Science ....... & 679 & 584 & 488 & 413 & 335 & 256 \\
\hline Space Applications $\ldots \ldots \ldots \ldots \ldots \ldots \ldots \ldots$ & 189 & 153 & 146 & 124 & 87 & 74 \\
\hline Manned Space Flight Operations, & & & & & & \\
\hline Apollo $\&$ advanced mission studies & 957 & 582 & 345 & 226 & 218 & 218 \\
\hline space shuttle $\ldots \ldots \ldots \ldots \ldots \ldots \ldots \ldots \ldots \ldots$ & 200 & 475 & 850 & 1,100 & 1,190 & 1,090 \\
\hline Space Research and Technology $\ldots \ldots \ldots \ldots \ldots$ & 65 & 65 & 65 & 65 & 65 & 65 \\
\hline Nuclear power and propulsion ..... & 17 & 4 & 4 & 4 & 4 & 4 \\
\hline Technology utilization & 4 & 4 & 4 & 4 & 4 & 4 \\
\hline Tracking \& Data Acquisition...$\ldots \ldots \ldots$ & 248 & 250 & 254 & 254 & 254 & 247 \\
\hline $\begin{array}{l}\text { Construction of facilities } \ldots \ldots \ldots \ldots \ldots \ldots \ldots \ldots \\
\text { Research } \& \text { program management (includes }\end{array}$ & 77 & 112 & 150 & 100 & 80 & 70 \\
\hline $\begin{array}{l}\text { Research \& program management (includes } \\
\text { pay for all NASA personnel) } \ldots . . \ldots \ldots \ldots\end{array}$ & 715 & 707 & 707 & 707 & 707 & 707 \\
\hline Total, budget plan & 3,302 & 3,107 & 3,167 & 3,139 & 3,072 & 2,852 \\
\hline Total, outlays & 3,062 & 3,136 & 3,231 & 3,219 & 3,145 & 3,043 \\
\hline
\end{tabular}

seriously affect the ability to perform the mission. After a program has. reached its peak funding year (generally about 2 years prior to launch) little money can be saved by a program cancellation.

Thus, although NASA has attempted to match future budget predictions and projected mission costs so as to maintain a logical and feasible program, the imposition of budget reductions encourages the cancellation of young programs; i.e., those that have not yet reached their peak funding and, therefore, involve smaller losses in "sunk" costs. The effect of a significant budget cut thus tends to be the elimination of new program starts, and hence generation of a void in the program six to ten years in the future.

During the past decade or so, despite sizable budget variations imposed by the Apollo Moon effort, solar system exploration programs have maintained a steady level of funding through fiscal year 1973. However, the implications of the projected restriction of the FY 1974 and subsequent fiscal year NASA budgets to an arbitrary level of approximately $\$ 3$ billion, as shown in Table 5 , would be to seriously curtail the space science effort from a level of \$679 million* in FY 1973 to only $\$ 256$ million* in 1978 . As indicated above, this could almost eliminate the potential new program starts to be discussed in the next section.

\footnotetext{
*Note: These figures include all Space Science, not just solar system exploration, but their trends are comparable.
}

Two major factors limit NASA's ability to accurately predict costs of space missions- the abovementioned long period of time over which each program remains a line item in the budget and the fact that solar system exploration demands implementation of a very high level of technology. Figure 45 shows the costs of the major planetary missions to date. Note that the Viking mission is split up into the orbiter and the lander. Clearly, the Viking and Surveyor programs, which represent the highest technology and most complex missions, were the most costly. The spin-stabilized Pioneer spacecraft are the most simple configuration and the three-axis-stabilized Mariner family falls in between.

The impact of technology and development uncertainties are most clearly illustrated in Figure 46. The costs of the Surveyor program, which represented the largest step forward, were the most seriously underestimated. The other high-technology programs were also underestimated. On the other hand, relatively low-technology programs, like the Mariner '69 and Pioneers 10 and 11, were more accurately estimated. The underestimates in the costs of these latter programs can probably be linked more to variability in market prices than to technology uncertainties. Certainly, if the city of New York is not capable of estimating the refurbishment costs of Yankee Stadium to better than a factor of two, NASA's ability to predict the cost of an eight year long space program within $30 \%$ should not be considered too inaccurate a forecast.

The problem of estimating (and minimizing) 


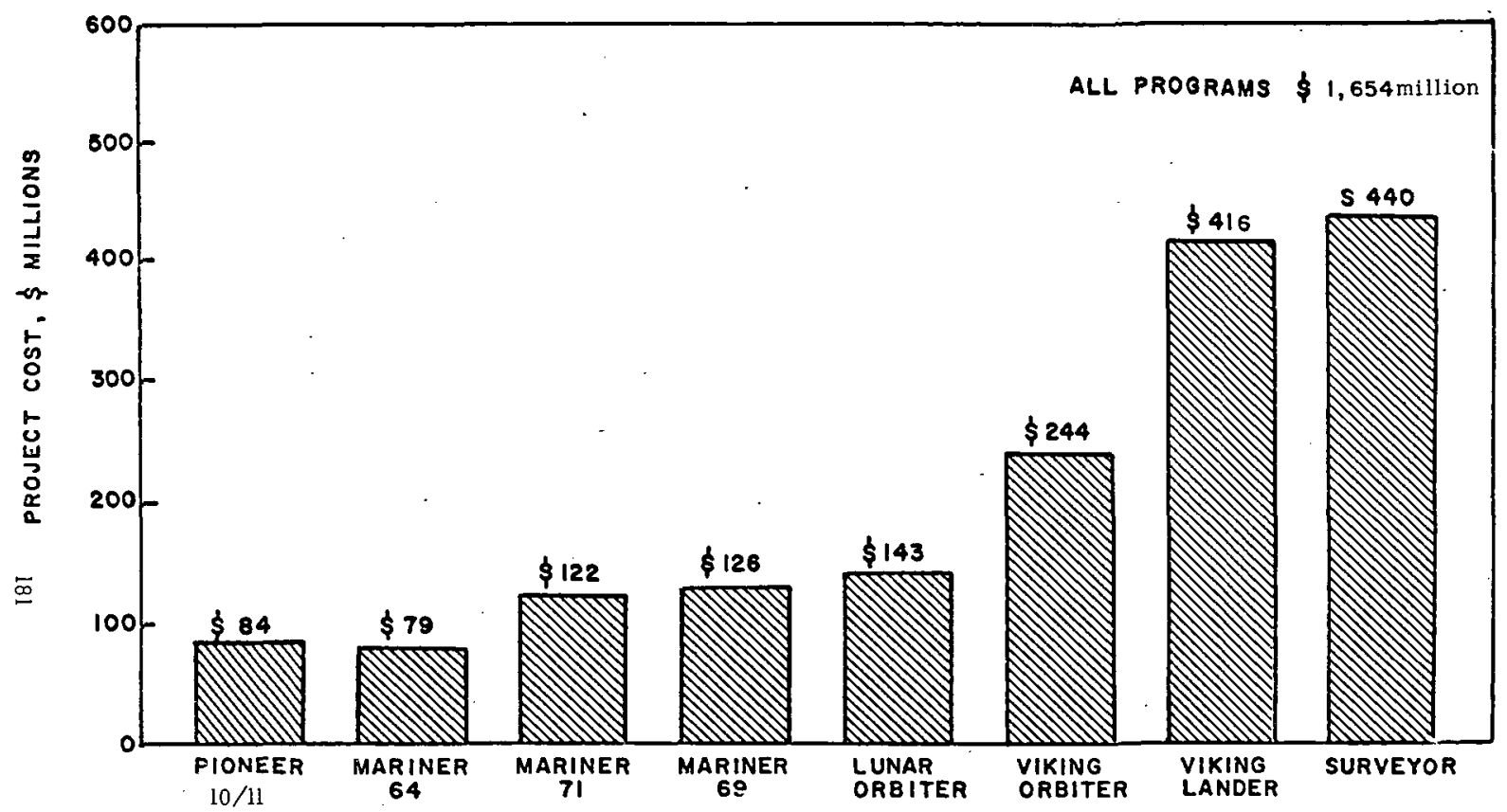

45 Project costs exclusive of NASA center project management.

costs for high technology programs requiring extremely high long-life reliability remains, however; and it is a serious problem. Since the early days of the Ranger program, which resulted in a string of six failures followed by a Congressional heaining, the United States solar system exploration effort has been remarkably successful; clearly, failures are undesirable, and to be avoided if at all possible. But to prevent failures altogether is impossible. A superhuman effort in quality control and testing can reduce failure rates to extremely low levels, but only with substantial impact on costs. What is necessary is a new approach that allows a limited number of failures, and by so doing, permits substantial reductions in quality control and testing costs in such a way as to (statistically) minimize the expected mission costs.

Current research dedicated to quantifying the risks and uncertainties associated with planetary missions will eventually permit the development of a decision-making policy to reduce the costs and increase the effectiveness of solar system exploration. These methodologies, when fully developed and accepted, should be able to provide better predictions of cost overruns due to unforeset $n$ technological problems.

Another economic constraint on solar system exploration arises from a recent trend to require all Federally sponsored technology programs to be justifiable on an economic basis; that is, the present value* of the benefits of a program should exceed the present value of the costs. Typically, the Federal government uses a discount rate of ten percent to compute present value, a rate which

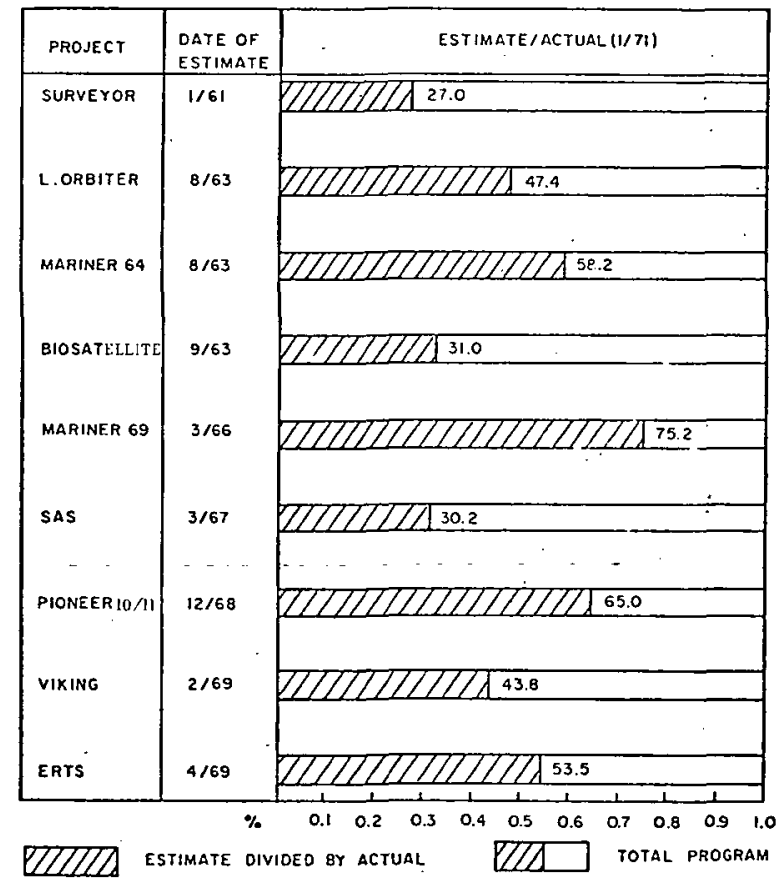

46 Past performance cost estimation.

has the effect of substantially diminishing any benefits that occur beyond about 10 years into the future. Furthermore, as the budget for advanced technology programs becomes significant, elected officials become interested in the direct

\footnotetext{
*Present value is the sum of the yearly benefits or costs with each year's value discounted to the present at an interest rate called the "discount rate."
} 
benefits these programs might provide to their electorate; e.g., "What has solar system exploration done for the people of my district?"

This point has been discussed from a general viewpoint in Chapter 2. The fact is that solar system exploration does not clearly offer benefits whose present value exeeds that of its costs at a ten percent discount rate. Neither does it offer short-term solutions to the energy crisis, the environmental crisis or the problems of our ghettos. Indeed, to the layman, there appears to be some dearth of economically supportable reasons for pursuing solar system exploration, at least within the constraints of the ten percent discount rate.

When we ask if there are alternatives to solar system exploration, in order to evaluate its costeffectiveness, we are faced with the simple answer that there are today no viable alternatives. Thus, it merely remains to decide whether or not to conduct such exploration; and in order to reach that decision, we must make cost-benefit comparisons between solar system exploration and other programs.

In making such comparisons, however, it is clearly improper to apply the same ten percent rate of discount to the analysis of research programs as we currently apply to short-term projects-applications of new technologies, for example, where ten percent is approximately the "cost of capital." The returns of long-term re- search programs such as solar system exploration are not guaranteed, and certainly are not of so short-term a nature that a fixed discount rate can be applied to them for purposes of comparison with other programs competing for funds. It is necessary, therefore, to approach these efforts with a certain measure of faith, and, as has been illustrated in Chapter 2 of this Review, there is good precedent for expecting that such faith is ultimately justified by more than commensurate benefits.

\section{E. The Program}

NASA's presently-conceived program for solar system exploration is summarized in Figure 47. The uppermost group in the figure (PL 1 through PL5) constitute the approved and ongoing programs; all the others are at most in the "study" phase. (Note, however, that several Earth-orbital missions capable of solar system exploration functions, such as the Large Space Telescope (LST), do not appear in the listing of Figure 47.) Missions already launched, as well as the other ongoing programs (the 1975 Viking orbiter and lander for the exploration of Mars, the 1974 and $1977^{\circ}$ Helios Sun-explorers, and the 1977 Jupiter/Saturn Mariners) have been discussed at length in previous sections.

The potential future missions under study (see Fig. 47) are capable of meeting all the objectives

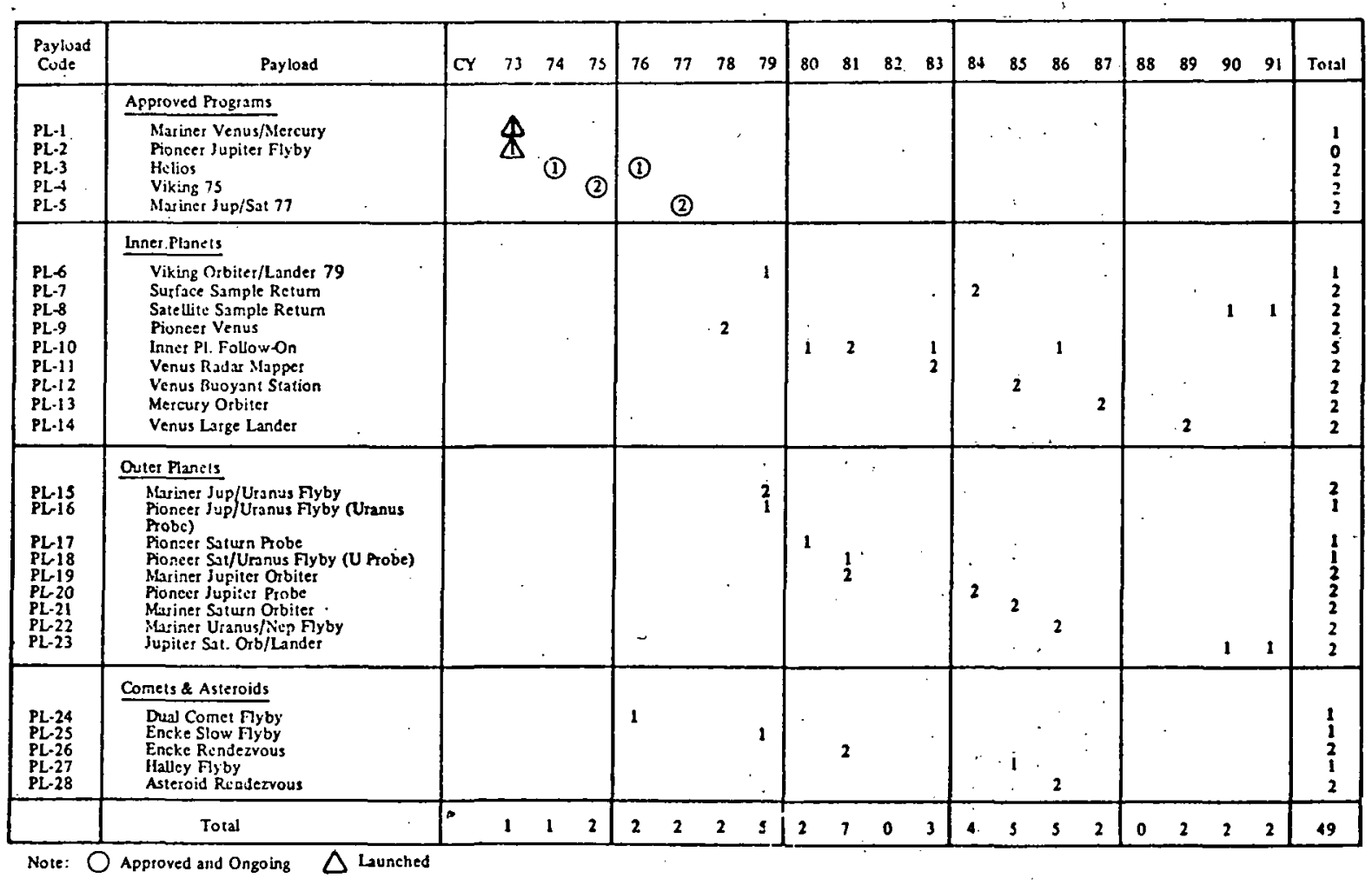

47 Planetary exploration programs under study (the numbers represent numbers of missions). 
outlined in this chapter. A second Viking orbiterlander in 1979 is being contemplated, as well as several sample-return missions for the further exploration of Mars. Venus is expected to receive attention from planetary-atmosphere probes to be carried by Pioneer spacecraft, a radar mapper, a buoyant atmospheric station, and a large lander. Five additional inner-planet missions are being studied for the 1980's, and missions to all of the outer planets except Pluto are being considered, with orbiters, flybys, probes, and landers as possibilities over the period from 1979 to 1991 . Flyby and rendezvous mission opportunities to comets and asteroids during the next 13 years are also included. Note that virtually all the planetary missions, including the currently flying Jupiter Pioneers and Venus/Mercury Mariner, also sample the interplanetary medium and its response to solar activity, as well as galactic phenomena such as cosmic radiation.

Other possibilities, although not specifically identified in the listing of Figure 47, include automated Mars rovers similar to the USSR's Lunokhod Moon-rover, as well as rendezvous, with a possible landing, on satellites of Mars, and, conceivably, Jupiter and/or Saturn.

Also, only a single cometary rendezvous mission is indicated, and only one to an asteroid, each using two spacecraft. However; because of the high scientific promise of missions to these small bodies, the Comet and Asteroid Mission Study Panel has recommended a coordinated program of flyby, rendezvous, lander, and sample-return missions:

"Obviously, some of the features of comets and asteroids could be observed from flybys. The more detailed studies, however, require a rendezvous with some time spent flying in close formation. A landing with in situ observations and high data rate communications would increase the exploration potential by a large margin. By far the highest information yield can be obtained from material brought back to Earth-bound laboratories for careful and detailed analyses with high precision methods. This value assessment of mission types is borne out by experience with the lunar landing program."

Because future expenditures for solar system exploration are likely to remain severely limited during these next two decades, it is essential that great attention be given to cost effectiveness. Future developments should be planned to take advantage of common features of several missions, so as to minimize development costs. For example, atmospheric-entry probe technology is similar for Venus and the outer planets (except for Jupiter, which presents a significantly more severe environment), and may well profit considerably from current research on the space shuttle. Standardized assemblies should be defined and developed for multiple use: star trackers, multimillion-bit storage devices, higher power long-life radio transmitters, and scientific instruments.

Planning must allow sufficient lead time and flexibility to permit revision and reshaping of future plans as we learn from current missions. For example, design of a Jupiter orbiter is highly dependent upon the intensity of Jupiter's radiation fields, but data by direct measurement from Pioneer 10's December 1973 flyby are only now being analyzed. Program adaptability should be emphasized so that uncertainties can be anticipated as a normal and healthy aspect of scientific exploration.

It is quite clear, however, that the severely limited future funding for space science activities which is implied by the current NASA (FY 1974) budget, as was illustrated earlier in Table 5 , is inadequate to support the level of activity indicated by the planetary exploration program shown in Figure 47. A decision is needed either to allow this significant program to dwindle away, thereby effectively foreclosing on whatever future potential benefits it may bring, or to support it at a level which permits it to survive and, hopefully, to flourish. 


\section{List of Tables}

1 Properties of the planets of our solar system.

2 Satellites of the solar system.

3 Bit rate capability.

4 Current and near-future launch vehicles used for NASA automated missions.

5 National Aeronautics and Space Administration-Funding implications of fiscal year 1974 budget.

\section{List of Figures}

1 A far-ultraviolet photograph of the Earth, including the atomic hydrogen Lyman-alpha line at 121.6 nanometers. This picture was obtained by the Naval Research Laboratory Far Ultraviolet Camera from the lunar surface during the Apollo 16 mission. The diffuse glow is due to the hydrogen geocorona, which is detectable out to a distance of more than 15 Earth radii from the Earth. A portion of the southern polar auroral zone is visible on the lower right edge of the Earth. (Courtesy Naval Research Laboratory)

2 Structure of the terrestrial magnetosphere. The incoming solar wind is deflected by the Earth's magnetic field; the limit of penetration is known as the magnetopause. The deflection of the supersonic solar wind causes a shock front to occur up-sun of the magnetopause. Within the shaded regions of the magnetosphere, charged particles can be trapped by the Earth's magnetic field. At the borders of these trapping zones, particles can precipitate into the atmosphere, causing the polar aurorae.

3 A sequence of data, obtained by a USAF meteorological satellite, showing the development of an auroral display over the northern polar regions. The figure is composed of six orbital passes, ordered from left to right. The lights of cities can be seen in the lower parts of each orbit of data.

4 A composite ultraviolet-light photograph of Venus taken by Mariner 10 on February 6, 1974. The - cloud patterns show. the general circulation of the upper atmosphere. The south ecliptic pole is in the bottom frame, and the morning terminator is at the right. (Courtesy NASA)

5 Variation of temperature with altitude in the Venus atmosphere, based on a combination of ground-based observations and probe measurements. On the right are shown the pressures corresponding to the different altitudes.
6 For a planet with no appreciable magnetic field, the solar wind can approach much closer to the planet-that is, to the point at which the solar wind pressure equals the atmospheric pressure (anemopause). Any ionized atmospheric constituents which cross upward across the anemopause boundary can be swept away by interactions with the solar wind.

7 Photographs of Mars obtained with the 200-inch Palomar telescope. Note the lower contrast of surface features in blue light compared to red light. (Courtesy The Hale Observatories)

8 Nix Olympica, a gigantic volcanic mountain on Mars, as photographed by the Mariner 9 orbiter in January 1972. The mountain is more than 500 kilometers ( 310 miles) across at its base, more than twice as broad as the largest volcanic pile on Earth (the island of Hawaii). Also its height, about $23 \mathrm{~km}$ ( 70,000 feet) above the surrounding plain, is more than twice that of Mt. Everest. (Courtesy NASA)

9 This 700-kilometer (440-mile) long sinuous valley, photographed by the Mariner 9 Mars orbiter, appears to have been eroded by some sort of flowing fluidindicating that perhaps liquid water existed on Mars in the past. (Courtesy NASA)

10 A closeup view of Phobos, the larger and closer of the two Martian satellites, obtained by Mariner 9 . (Courtesy NASA).

11 A photograph of the planet Jupiter, made with the 200-inch Palomar telescope. Taken in blue light, this photograph shows the banded structure of the cloud layer and the Great Red Spot. (Courtesy The Hale Observatories)

12 Diagram of the structure of Jupiter's atmosphere, showing the theoretically-expected variation of temperature and pressure with depth, 
and the altitudes and compositions conjectured for the cloud layers.

13 Hypothetical configuration of the magnetosphere of Jupiter prior to the analysis of Pioneer-10 data. Note that Jupiter's magnetosphere is much larger, even relative to the size of the planet, than that of the Earth, and that it includes the four major satellites. Pioneer 10 measurements showed that the magnetopause might be as much as 100 Jupiter $\operatorname{radii}(\mathrm{Rj})$ ahead of the planet, twice as far as is shown in this figure, and that the magnetopause is much more.complex than that shown here.

14 A photograph of the planet Saturn, obtained with the 200-inch Palomar telescope. (Courtesy The Hale Observatories)

15 Atmospheric temperature profiles and cloud models of Jupiter, Saturn, Uranus, and Neptune, showing the condensation threshholds of methane $\left(\mathrm{CH}_{4}\right)$, ammonia $\left(\mathrm{NH}_{3}\right)$, ammonium hydrosulfide $\left(\mathrm{NH}_{4} \mathrm{SH}\right)$, water $\left(\mathrm{H}_{2} \mathrm{O}\right)$, and ammonia in aqueous solution $\left(\mathrm{H}_{2} \mathrm{O}+\mathrm{NH}_{3}\right)$.

16 A photograph of Comet Tago-Sato-Kosaka. which appeared in 1970. (Courtesy Cerro Tololo International Observatory)

17 A composite of a photograph of the solar corona, taken during a total eclipse, and of the disk of the Sun out of eclipse near that time. Note the correlation between coronal plumes and active areas on the solar surface. (Courtesy NOAA)

18 Schematic diagram of the Archimedean-spiral structure of the interplanetary magnetic field in the ecliptic plane.

19 A diagram of the heliosphere. The region of supersonic solar wind is not symmetrical, being closer to the Sun in the direction from which the interstellar gas is approaching. The ionized component of the interstellar gas is excluded from the heliosphere, and if the relative velocity is supersonic, a "bow shock" may be formed as indicated by the dashed line.

20 Blocking of radiation by the atmosphere, from radio to gamma-ray wavelengths.

21 Large space telescope.

22 Pioneer spacecraft characteristics.

23. Pioneer 10 and 11 spacecraft configurations.

24 Mariner spacecraft characteristics

25 Mariner Venus-Mercury 1973 spacecraft.

26 Venus-Mercury spacecraft experiments and subsystems.
27 The Mariner Jupiter-Saturn 1977 spacecraft encounters Saturn.

28 Mariner Jupiter-Saturn 1977 spacecraft.

29 Viking orbiter-lander spacecraft approaching Mars.

30 Artist's conception of Viking lander on the Martian surface.

31 Viking lander science configuration.

32 Exploded view of Viking spacecraft.

33 Viking spacecraft configuration, showing both orbiter and lander.

34 Model of Helios spacecraft.

35 Helios spacecraft, showing principal elements.

36 Titan III-E/Centaur expendable launch vehicle.

37 Space shuttle configuration

38 Solar electric propulsion stage (SEP) conceptual definition.

39 Solid-core nuclear rocket engine.

40 Grand Tour gravity-assist opportunities in late 1970's. Trajectory A shows how a single spacecraft could flyby Jupiter, Saturn, and Pluto; in B, Jupiter, Uranus, and Neptune.

41 Mariner Venus-Mercury flight trajectory.

42 Prime and backup landing sites for Viking spacecraft.

43 Pioneer flight path to Jupiter.

44 The Helios mission will take the spacecraft within about 28 million miles of the Sun, the closest approach to the solar body by a man-made vehicle.

45 Project costs exclusive of NASA center project management.

46 Past performance cost estimation.

47 Planetary exploration programs under study (the numbers represent numbers of miss ions). 
accelerometer

\section{angular \\ momentum}

aphelion

apparition (of

a comet)

asteroid belt

astronomical unit

\section{atmospheric probe \\ attitude \\ control}

ballistic
flyby
bar

bit

bow shock
wave
celestial
mechanics

charged particle detector

charged particle telescope

corona device for measuring rate of change of velocity (acceleration), usually in either direction along a particular line or axis, by means of a suspended inertial mass.

a measure of a body's tendency to continue rotating at a particular rate (or at rest) around a particular axis; obtained by multiplying the moment of inertia of a body by its angular speed.

in an orbit around the Sun, the point farthest out; opposite of perihelion.

the period of time when a comet becomes visible on a photographic plate.

a solar-orbit zone or doughnut-like ring located between 2.1 and 3.5 astronomical units in which are, found thousands of asteroids (also called minor planets or planetoids) of irregular shapes and diameters from a fraction of a mile to 500 miles.

a measure of solar-system distance equal to the average distance between Earth and Sun; its value is approximately $149,000,000 \mathrm{~km}(93,200,000$ miles $)$.

scientific device (usually carried by a spacecraft) for determining the pressure, composition, and temperature of a planet's atmosphere at different altitudes.

process of maintaining or changing a spacecraft's orientation in space (usually with gas jets) so that solar panels and other insturments can be pointed at target bodies.

unpowered flight similar to a bullet's trajectory, governed by gravity and by the body's previously acquired velocity.

unit of atmospheric pressure, divided into 1,000 millibars; pressure at Earth's surface is about one bar, at Mars' surface less than 10 millibars, and at Venus' surface about 90 bars.

unit of information equivalent to the result of a choice between two equally probable alternatives.

the interface formed where the electrically charged solar wind encounters an obstacle in space such as the atmosphere or magnetic field of a plane. Behind the shock wave, the solar wind speed is subsonic.

dynamic relationships existing among bodies of the solar system; description of the relative motions of celestial bodies under the influence of their mutual gravitational attractive forces.

a device which counts and/or measures the energy of electrically charged particles (electronic, protons, alpha particles, larger ions) in space.

a group of charged particle detectors used to measure the direction of particle passage, as well as for counting the number of such particles.

the glowing outer reaches of the Sun's luminous, active gaseous envelope, visible during a total eclipse; includes towering prominences and shades off into the invisible, tenuous solar wind (plasma) which streams out into the solar system. 
cosmic dust

cosmic ray

diffraction-limited

ecliptic

\section{electromagnetic radiation}

encounter

entry probe

exóbiology

flyby

Galileañ satellites

gas

chromátograph

geomagnetic

storm

Grand Tour

gravity assist

heliosphere

imaiging photopolarimeter

infrared radiometer

inner planet

in terplanetary medium fine microscopic particles adrift in space; sometimes called micrometeorites.

charged particles similar to the solar wind but traveling from interstellar space into the solar system at extremely high energies (speeds).

limited in resolution by the wave-length of light.

the plane of motion defined by Earth's orbit around the Sun in space.

energy transmitted through space in any of the following forms: radio waves, infrared radiation, radiant heat, visible light, ultraviolet rays, $x$-rays, or gamma rays.

a close flyby or rendezvous of a spacecraft with a target body.

(see atmospheric probe).

the study of extraterrestrial environments for living organisms, recognition of evidence for possible existence of life in these environments, and study of any nonterrestrial life that may be found.

space mission in which instrumented vehicle passes a planet without going into orbit, entering atmosphere, or landing on the surface.

the four large satellites of Jupiter: Callisto, Ganymede, Europa, and Io; discovered by Galileo in 1609.

device for organic chemical analysis in which the unknown mixture is vaporized and blown through a filtering column which holds back different organic compounds at different rates, thus separating them at the outlet.

sudden worldwide fluctuations in Earth's magnetic field, associated with solar flare-generated shock waves which propagate from the Sun to the Earth.

a spacecraft mission proposed for the late 1970's to fly past three or four of the outer planets when favorably aligned; it would use the moving gravity fields of Jupiter and Saturn to alter the speed and direction of flyby spacecraft in flights to Uranus and Neptune, or to Pluto.

change in a spacecraft's velocity and direction achieved by calculated flyby through a planet's gravitational field without use of supplementary propulsive energy.

the region in the solar system occupied by the Sun's corona, including the solar wind, which is known to extend beyond Earth.

sensor for measuring brightness and polarization of light; usually mounted to scan target so that readings can be assembled into a picture.

instrument which measures the temperature of an object from the intensity of radiated heat.

Mercury, Venus, Earth, and Mars (see "terrestrial planets").

the environment of charged particles and associated magnetic fields existing in the solar system outside the regions affected by the atmosphere, ionosphere, or radiation belts that envelop individual planets. 


\section{interstellar medium}

ion

ionosphere

lander

magnetometer

magnetopause

magnetosheath

magnetosphere

mascon

mass

spectrometer

neutral

atmosphere

newton

occultation

orbiter

outer planets

parabolic

antenna

parking orbit

particles
and fields

perihelion

65 the environment of charged particles and dust that exists in the Milky Way Galaxy throughout the region between the stars.

electrically charged atomic particle.

electrically charged upper layer in an atmosphere which is ionized by the Sun's ultraviolet and $\mathrm{x}$-radiations.

spacecraft or mission which lands on another celestial body; e.g., Surveyor on the Moon, Viking on Mars.

device for measuring the strengths of magnetic fields.

the outer boundary of the magnetosphere.

the region of disturbed solar wind which lies between the bow shock wave and the magnetopause.

the region (not actually spherical) within which the magnetic field of a planet is confined by the solar wind.

area of mass concentration or high density within a planetary body, usually near the surface.

device that separates a stream of charged particles into a spectrum according to the masses of the particles; used for measuring the atomic masses.

that portion of an atmosphere consisting of atoms and molecules, not electrically charged ions.

metric unit of force, equivalent to 0.2247 pounds:

a blocking action, as when the Moon passes between a star and an observer. cutting off its light; a planet with an atmosphere gradually cuts off light or radio waves from a spacecraft and the occulting atmosphere can be studied by this means.

spacecraft or mission involving insertion of vehicle into orbit about another celestial body.

Jupiter, Saturn, Uranus, Neptune, and Pluto; the first four differ radically from Earth and the other terrestrial planets.

a radio reflector used for radar, microwave, and space communications; in receivers the reflecting surface reflects parallel beams to a single focal point, where the active element of the antenna is located; in transmitters the reflecting surface converts the source signal to a parallel beam.

a temporary orbit (usually around Earth) in which a space vehicle coasts or "parks" between intervals of powered flight before injection into a transfer trajectory to another body in the solar system.

cosmic dust, plasma, other charged particles including cosmic rays, and magnetic fields; the term usually refers to instruments and experiments conducted in interplanetary space.

in a solar orbit, that point in the ellipse closest to the Sun. 
plane of
the ecliptic

plasma

plasma detéctor

plasma science experiment

proton

radar altimeter

radar astronomy

\section{radioisotope \\ generator}

radio telescope

rendezvous

rover

S-band

solar electric propulsion

solar wind

spectral survey

sublimation

terminal

guidance

terminator see ecliptic

an ionized gas containing about equal numbers of positive ions and electrons, as in the solar wind.

device for measuring the amount and/or velocity and direction of solar plasma.

analysis of data on ion energies and directions measured by means of plasma detectors.

a positively charged atomic particle; one proton constitutes the nucleus of the hydrogen atom.

device for measuring range or distance; for example, from an approaching spacecraft by timing the travel of a radar pulse down to the surface and back.

study of the motion and form of other planets with powerful and precise radar equipment; differs from radio astronomy in that signals are transmitted by the observer and reflected by the object of interest.

a source of electrical power in which the energy liberated in radioactive decay is collected as heat and converted, usually directly by means of thermocouple action, into electricity.

a precise and sensitive radio receiving system using a parabolic or other highly directional antenna to locate and track radio sources in the sky.

a space mission in which the spacecraft is maneuvered so as to fly alongside a target body, such as a comet or asteroid, at zero relative velocity.

a roving vehicle, either manned or remotely controlled, for planetary or lunar surface exploration.

a radio-frequency band between 1,550 and 5,200 megahertz (VHF and UHF television signals have frequencies between 50 and 900 megahertz).

a relatively low-thrust, long-continuing method of propulsion in which stored matter, called reaction mass, is given very high velocity and jetted out by means of electrical energy generated from solar panels.

plasma blown constantly at supersonic speed out of the Sun in all directions; consists of electrons, protons and alpha particles (hydrogen and helium atomic nuclei, both positively charged), and some heavier ions.

measurements of the wavelengths or energies of radiation emitted by a given source or from all accessible regions of the celestial sphere.

change of state directly from the solid to the gaseous phasé.

navigation of a spacecraft, usually during its approach to a planet, by observing the angular position and motion, and perhaps also the apparent size of the target body.

the shadow line around a planet or satellite which separates the sunlit from the shaded side. 
terrestrial

planets

thermocouple action

transfer

trajectory

trapped

radiation

ultraviolet

airglow

spectrometer

ultraviolet spectrometer

X-band

zodiacal light
Mercury, Venus; Earth, and Mars; the planets in the inner solar system which fall in a class with our own as to size and density.

the process whereby, in an electrical circuit made of two dissimilar metals or semiconductors, with one junction heated and the other cold, an electric current is generated (see radioisotope generator).

that part of a spacecraft's travel in space between, usually, Earth, and a target body; usually unpowered or purely ballistic.

charged particles of moderately high energy, trapped by a planet's magnetic field.

an ultraviolet spectrometer designed especially to define the faint fluorescent glow of ultraviolet light in a planetary atmosphere in order to study the gases or ions and energy sources which produce it.

an optical instrument for analyzing the intensity of ultraviolet light at various wavelengths.

a radio-frequency band from 5,200 to 10,900 megahertz, designated originally for high-frequency radar; now being used in spacecraft radio propagation experiments for space communications.

a faint glow around the general region of the plane of the solar system; thought to be sunlight reflecting from particles of cosmic dust found mostly in this plane. 

National Aeronautics and Space Administration 